\title{
A GENERALIZED CONTOU-CARRĖRE SYMBOL AND ITS RECIPROCITY LAWS IN HIGHER DIMENSIONS
}

\author{
OLIVER BRAUNLING, MICHAEL GROECHENIG, AND JESSE WOLFSON
}

\begin{abstract}
We generalize Contou-Carrère symbols to higher dimensions. To an $(n+1)$-tuple $f_{0}, \ldots, f_{n} \in A\left(\left(t_{1}\right)\right) \cdots\left(\left(t_{n}\right)\right)^{\times}$, where $A$ denotes an algebra over a field $k$, we associate an element $\left(f_{0}, \ldots, f_{n}\right) \in A^{\times}$, extending the higher tame symbol for $k=A$, and earlier constructions for $n=1$ by Contou-Carrère, and $n=2$ by Osipov-Zhu. It is based on the concept of higher commutators for central extensions by spectra. Using these tools, we describe the higher Contou-Carrère symbol as a composition of boundary maps in algebraic $K$ theory, and prove a version of Parshin-Kato reciprocity for higher ContouCarrère symbols.
\end{abstract}

\section{INTRODUCTION}

This article concerns a higher-dimensional generalization of the Contou-Carrère symbol CC94. The original symbol plays a key role in the local theory of generalized Jacobians for a relative curve, as developed by Contou-Carrère [CC79], [CC90]. This theory was inspired by a conjectural picture due to Grothendieck Gro01]. If the relative curve is just a plain curve over a field, the symbol specializes to the tame symbol. We review this in detail along with an explicit definition below in 1.5. But in general the Contou-Carrère symbol is far richer. For example, one recovers the residue symbol in its tangent space. This aspect cannot be seen in the tame symbol.

If $G$ : Rings $\rightarrow$ Groups is a group functor, one defines its (formal) loop group $L G$ as the group functor

$$
L G(A):=G(A((T))), \quad \text { where } \quad A((T)):=A[[T]]\left[T^{-1}\right] .
$$

The classical Contou-Carrère symbol is a non-degenerate pairing of loop groups

$$
L \mathbb{G}_{m} \times L \mathbb{G}_{m} \longrightarrow \mathbb{G}_{m},
$$

Received by the editors November 12, 2015, and, in revised form, December 17, 2016, November 19, 2018, May 25, 2019, and September 28, 2020.

2020 Mathematics Subject Classification. Primary 19D45.

The first author was supported by DFG SFB/TR 45 "Periods, moduli spaces and arithmetic of algebraic varieties", the Alexander von Humboldt Foundation, and DFG GK1821 "Cohomological Methods in Geometry" . The second author was partially supported by EPRSC Grant No. EP/G06170X/1. The third author was partially supported by an NSF Graduate Research Fellowship under Grant No. DGE-0824162, by an NSF Research Training Group in the Mathematical Sciences under Grant No. DMS-0636646, and by an NSF Post-doctoral Research Fellowship under Grant No. DMS-1400349. This research was supported in part by NSF Grant No. DMS-1303100 and EPSRC Mathematics Platform grant EP/I019111/1. 
which can also be seen as the statement that $L \mathbb{G}_{m}$ is self-dual under Cartier duality. Our generalized symbol will be $(n+1)$-multilinear on $n$-fold loops

$$
\underbrace{L^{n} \mathbb{G}_{m} \times \cdots \times L^{n} \mathbb{G}_{m}}_{n+1 \text { factors }} \longrightarrow \mathbb{G}_{m}
$$

for any $n \geq 1$. This might at first sight not look like an appropriate generalization of a duality, but we shall explain below both why the generalization should have this form, as well as our approach for defining it. See Theorem 1.1 if you want to jump ahead to a precise formulation of the properties of our symbol (including compatibility with the classical Contou-Carrère symbol and with a previous construction of a two-dimensional Contou-Carrère symbol in [OZ16]), or jump to Theorem 1.4 for the reciprocity law which we prove for it, generalizing the reciprocity law of the Contou-Carrère symbol on curves. Even when speaking of the classical ContouCarrère symbol, the literature approaches the topic from various angles and we use this introduction as an opportunity to explain the relations between these viewpoints. This is also vital to explain the idea behind our construction in arbitrary dimension.

1.1. The origins. Let us first review the classical story before Contou-Carrère's theory. Suppose (for simplicity) that $X / k$ is a smooth curve over an algebraically closed field $k$, not necessarily proper. The curve comes equipped with a generalized Jacobian $J$ along with an Abel-Jacobi map

$$
X \longrightarrow J
$$

sending a closed point $x$ to the degree onf 1 line bundle $\mathcal{O}([x])$. There are many ways to formulate geometric class field theory, but a reasonable summary can be given in terms of the following two principles:

Every morphism $X \rightarrow G$ to a commutative algebraic $k$-group $G$ factors uniquely over $J$. This can be phrased as an isomorphism

$$
\underset{k-\text { groups }}{\operatorname{Hom}}(J, G) \cong H^{0}(X, G),
$$

where we consider fppf cohomology on the right side. This is essentially characterizing $J$ as a type of Albanese variety 2 Moreover, extensions of $J$ by $G$ correspond to $G$-torsors,

$$
\underset{k-\text { groups }}{\operatorname{Ext}}(J, G) \cong H^{1}(X, G) .
$$

This property provides a link to class field theory: As a special case of it, one obtains that every abelian finite étale covering of $X$ arises as the pullback of an isogeny of the Jacobian. For example, if $X$ is $\mathbb{P}^{1}$ minus at least two points, the Jacobian has a non-trivial torus part and a pullback of the isogeny $\mathbb{G}_{m} \stackrel{\cdot n}{\rightarrow} \mathbb{G}_{m}$ yields a degree $n$ cyclic Kummer extension. The kernel sequence of this isogeny defines the corresponding extension in Ext $^{1}$.

\footnotetext{
${ }^{1}$ Other people prefer to fix an auxiliary point $p$ and take differences $[x]-[p]$ so that one obtains a degree zero line bundle, living in what is perhaps more classically called the Jacobian or $\mathrm{Pic}^{0}$. The dependency on the choice of $p$ makes this less functorial. We use the term "Jacobian" in a broader sense here.

${ }^{2}$ In a generalized sense.
} 
There is a more precise formulation, where one replaces $J$ by a Jacobian with respect to a fixed modulus $\mathfrak{m}$ with support in $\bar{X} \backslash X$, and then one obtains a description of exactly such abelian finite coverings which are étale over $X$ and whose ramification at the boundary $\bar{X} \backslash X$ is bounded by the multiplicities of $\mathfrak{m}$.

Background can be found in Ser88, but our exposition here follows AGV71, Tome 3, Exposé XVIII] and [BE01, Appendix, Deligne's letter, (e)] 4

1.2. The relative situation. Contou-Carrère generalized this story to the situation of relative curves, i.e. the compactified curve $\bar{X} / k$ is replaced by a flat morphism of finite presentation

$$
f: \bar{X} \longrightarrow S
$$

such that the fibers are geometrically integral of dimension one and locally projective over the base and $X:=\bar{X} \backslash D$ is taken to be the open complement of a relative divisor $D$. The papers [CC79], CC90] set up a corresponding theory of a relative generalized Jacobian attached to $f$, along with a local theory [CC81]. The analogue of Equation (2) is set up in [CC13, Thm. 1.6.6].

The present paper also concerns the relative situation, but we should first explain a few more concepts in a simpler setting 5

1.3. Local symbols and the Contou-Carrère symbol. Returning to the original formulation of class field theory for curves, i.e. back in the situation $S:=\operatorname{Spec} k$ with $k=\bar{k}$, one can also understand abelian finite étale coverings with bounded ramification using a more classical approach based on the idèle class group and methods adapted from number theory 6

In terms of the idèle class group, the choice of a modulus $\mathfrak{m}$ bounding the allowed ramification identifies the deck transformation group with a so-called ray class group. Such is a quotient of the idèle class group. The fact that the global reciprocity map is trivial on the terms which we quotient out, amounts to a reciprocity law. Since the global reciprocity map is a product of the local reciprocity maps, the triviality of the global action means that a suitable linear combination of local terms adds up to zero. Neglecting a few details, these contributions amount to the so-called local symbols. The formalism of local symbols extends beyond the mere application in class field theory to all commutative algebraic $k$-groups $G 7$

For example, the tame symbol is a local contribution which arises in the context of Kummer cyclic coverings. These abelian extensions arise as the pullback along an isogeny $\mathbb{G}_{m} \stackrel{\cdot n}{\rightarrow} \mathbb{G}_{m}$, just as mentioned above.

\footnotetext{
${ }^{3}$ It is standard to call this a modulus in this setting, but in this context it is the same thing as an effective Weil divisor. The Jacobian $J_{\mathfrak{m}}$ classifies line bundles with extra trivializations at the support of the modulus. Sections of such can be understood in terms of certain lattices; a concept we shall soon return to in $\$ 1.9$

${ }^{4}$ Recently, it has become more popular to re-interpret geometric class field theory as rank one local systems arising as pullbacks from the Jacobian. We refrain from using this slight shift of perspective in this text.

${ }^{5}$ For the sake of completeness, we mention that Deligne Del91 has also found the ContouCarrère symbol, albeit in an analytic setting. This extends the overall picture in a different direction and would lead us too far here.

${ }^{6}$ That is: Approaches to the global class field theory of curves which do not rely on the Jacobian (there are several ways to do this).

${ }^{7}$ This theory has since found a new formulation in terms of reciprocity sheaves [R17, [KSY16] or more broadly motives with modulus.
} 
This suggests the existence of a local analogue of the entire story, where the roles of $X, \bar{X}$ are replaced by

$$
X:=\operatorname{Spec} \operatorname{Frac} \mathcal{O}_{X, x} \quad \text { and } \quad \bar{X}:=\operatorname{Spec} \mathcal{O}_{X, x},
$$

so that one can think of $X$ as a punctured disc and $\bar{X}$ the "compactification" obtained by filling the puncture. This setting would still retain most of the global geometry since the field of fractions of course determines the curve (so it is not 'as local' as one might wish for). This suggests to work with the formal completions instead.

$$
X:=\operatorname{Spec} \operatorname{Frac} \widehat{\mathcal{O}}_{X, x} \quad \text { and } \quad \bar{X}:=\operatorname{Spec} \widehat{\mathcal{O}}_{X, x} .
$$

Of course, one can choose a local coordinate and obtain (non-canonical) isomorphisms

$$
\operatorname{Frac} \widehat{\mathcal{O}}_{X, x} \simeq \kappa(x)((t)) \quad \text { and } \quad \widehat{\mathcal{O}}_{X, x} \simeq \kappa(x)[[t]] .
$$

The analogy to the loop group construction in Equation (11) is apparent.

Before we continue, let us recall that these (formal) local contributions admit a class field theory in their own right, known as local class field theory.

1.4. Duality formulation of local class field theory. Let us first look at the original local theory originating from arithmetic. Suppose $F$ is a finite extension of $\mathbb{Q}_{p}$. Local class field theory can be expressed as a duality in Galois cohomology. The pairing

$$
H^{i}(F, \mathbb{Z} / n(1)) \otimes H^{2-i}(F, \mathbb{Z} / n) \longrightarrow H^{2}(F, \mathbb{Z} / n(1)) \cong \mathbb{Z} / n
$$

is non-degenerate for any $n \geq 1$ and any $i$. Here $\mathbb{Z} / n(1)$ refers to the Tate twist; one could also write $\mu_{n}$. Since

$$
H^{1}(F, \mathbb{Z} / n)=\operatorname{Hom}\left(\operatorname{Gal}\left(F^{\text {sep }} / F\right), \mathbb{Z} / n\right)
$$

is the $\mathbb{Z} / n$-dual of the abelianized Galois group, this encodes the classification of degree $n$ abelian étale coverings of Spec $F$ in terms of $H^{1}(F, \mathbb{Z} / n(1)) \cong F^{\times} / n F^{\times}$.

The same is true if $F$ is a finite extension of $\mathbb{F}_{p}((t))$, except that a more involved formulation is necessary if $p \mid n$, which we do not wish to discuss in the introduction (to keep this exposition at reasonable length) 8

Let us now discuss a generalization of this which is vital for understanding the deeper motivations for the present paper.

The above duality formulation of local class field theory can be generalized to $r$-local fields, e.g., when $F$ is a finite extension of

$$
\mathbb{Q}_{p}\left(\left(t_{1}\right)\right)\left(\left(t_{2}\right)\right) \ldots\left(\left(t_{r-1}\right)\right) \quad \text { or } \quad \mathbb{F}_{p}\left(\left(t_{1}\right)\right)\left(\left(t_{2}\right)\right) \ldots\left(\left(t_{r}\right)\right) .
$$

There are more $r$-local fields than just these, but again let us sweep this under the rug for the purpose of this introduction.

A duality formulation of class field theory as in Equation (6) remains intact also in this broader setting, but the cohomological dimension increases from 2 to $r+1$. We get, again ignoring the case where the characteristic divides $n$, a non-degenerate pairing

$$
H^{i}(F, \mathbb{Z} / n(i)) \otimes H^{r+1-i}(F, \mathbb{Z} / n) \longrightarrow H^{r+1}(F, \mathbb{Z} / n(i)) \cong \mathbb{Z} / n
$$

\footnotetext{
${ }^{8}$ The story is entirely analogous to what happens in geometric class field theory, where $\mathbb{G}_{a}$ (or truncated Witt vectors) are needed as the relevant commutative group scheme, and the pullbacks are Artin-Schreier-Witt extensions.
} 
Letting $i:=r$, this now pairs the $\mathbb{Z} / n$-dual of the abelianized Galois group with the cohomology group

$$
H^{r}(F, \mathbb{Z} / n(r)) \cong K_{r}^{M}(F) / n,
$$

where the map is the norm residue isomorphism. We observe two key facts: (1) as the cohomological dimension increases, the duality moves to higher homological degrees, and (2) the role of $\mathbb{G}_{m}$ in 1-dimensional class field theory is now taken over by a Milnor $K$-group (or the motivic sheaf $\mathbb{Z}(r)$, but let us stay entirely in the language of $K$-theory; see Remark 2.2).

1.5. Back to the Contou-Carrère symbol. The duality considerations in $\$ 1.4$ were only on the level of Galois cohomology, or the étale topos if you will. They are not geometric. Despite the formal similarity to Poincáre duality, the underlying scheme is just $\operatorname{Spec} F$ and the duality a group-theoretic fact of $\operatorname{Gal}\left(F^{\mathrm{sep}} / F\right)$. One would expect more, especially when attempting to move this story to the relative setting of $§ 1.2$. This is the motivation for the original Contou-Carrère symbol CC94.

We return to the situation of a relative curve. The Contou-Carrère symbol is a non-degenerate pairing on the loop group of $\mathbb{G}_{m}$.

$$
(-,-): L \mathbb{G}_{m} \times L \mathbb{G}_{m} \longrightarrow \mathbb{G}_{m}
$$

It can be given by an explicit formula. Using a presentation (10)

$$
f=\prod_{-\infty}^{i=-1}\left(1-a_{i} t^{i}\right) a_{0} t^{\nu(f)} \prod_{i=1}^{\infty}\left(1-a_{i} t^{i}\right), \quad \text { and } \quad g=\prod_{-\infty}^{i=-1}\left(1-b_{i} t^{i}\right) b_{0} t^{\nu(g)} \prod_{i=1}^{\infty}\left(1-b_{i} t^{i}\right)
$$

for suitable $\nu(f), \nu(g) \in \mathbb{Z}$ (just the order of the power series) and $a_{i}, b_{i} \in A$ (nilpotent for $i$ negative), the value is given by

$$
(f, g)=(-1)^{\nu(f) \nu(g)} \frac{a_{0}^{\nu(g)} \prod_{i=1}^{\infty} \prod_{j=1}^{\infty}\left(1-a_{i}^{j /(i, j)} b_{-j}^{i /(i, j)}\right)^{(i, j)}}{b_{0}^{\nu(f)} \prod_{i=1}^{\infty} \prod_{j=1}^{\infty}\left(1-a_{-i}^{j /(i, j)} b_{j}^{i /(i, j)}\right)^{(i, j)}}
$$

We can directly connect this to the local class field theory story of $\$ 1.4$. If we evaluate the Contou-Carrère symbol on a field $k$, it simplifies to

$$
\begin{aligned}
L \mathbb{G}_{m}(k) \times L \mathbb{G}_{m}(k) & \longrightarrow \mathbb{G}_{m}(k) \\
k((t))^{\times} \times k((t))^{\times} & \longrightarrow k^{\times}
\end{aligned}
$$

sending

$$
(f, g) \longmapsto(-1)^{v(f) v(g)}\left(\frac{f^{v(g)}}{g^{v(f)}}\right)(0) .
$$

Here we exploit that since the fraction in the big brackets has degree zero, its evaluation at zero is possible and non-zero. This expression is known as the tame symbol. Its relation to local class field theory is as follows: Taking $F:=k((t))$ for any field $k$ such that $\operatorname{char}(F) \nmid n$, the Galois cohomology pairing

$$
H^{1}(F, \mathbb{Z} / n(1)) \otimes H^{1}(F, \mathbb{Z} / n(1)) \longrightarrow H^{2}(F, \mathbb{Z} / n(2))
$$


can, through the norm residue isomorphism (as in Equation (9) ) be realized as a quotient of the natural pairing in Milnor $K$-theory

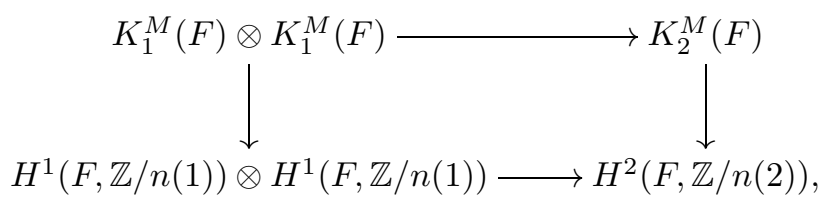

and along with the boundary map

$$
K_{1}^{M}(F) \otimes K_{1}^{M}(F) \longrightarrow K_{2}^{M}(F) \stackrel{\partial}{\longrightarrow} k^{\times},
$$

the composition of maps in the top row is given by the same formula as in Equation (13). This shows that the duality maps which occur in local class field theory are at least close to the ones realized by the tame symbol; and thus are reasonable to generalize in some way to the Contou-Carrère symbol. We also get a strong hint of the relevance of $K$-theory here. The full story is a little more complicated: The pairing in Equation (14) has a different Tate twist than in Equation (6), so it is a little bit off. Once the field contains a primitive $n$-th root of unity, one can pick an isomorphism of étale sheaves $\mathbb{Z} / n \simeq \mathbb{Z} / n(1)\left(=\mu_{n}\right)$ to fix this, but really the tame symbol corresponds to the (prime to the characteristic part of the) Hilbert symbol and not to the reciprocity pairing. Let us sweep these issues under the rug for the purpose of this introduction.

The boundary map $\partial$ in Equation (16) arises from the localization sequence in (Quillen) $K$-theory, corresponding to the open-closed complement decomposition

$$
\operatorname{Spec} k \underset{\text { closed }}{\hookrightarrow} \operatorname{Spec} \mathcal{O}_{F} \underset{\text { open }}{\longleftrightarrow} \operatorname{Spec} F
$$

Here $\left(\mathcal{O}_{F}, \mathfrak{m}\right)$ is the ring of integers in the local field $F$ and we use that $\mathcal{O}_{F} / \mathfrak{m}=k$. The boundary map appears in the attached long exact sequence in the spot

$$
\cdots \longrightarrow K_{2}\left(\mathcal{O}_{F}\right) \longrightarrow K_{2}(F) \stackrel{\partial}{\longrightarrow} K_{1}(k) \longrightarrow \cdots .
$$

In these low degrees there is no difference between Quillen $K$-theory and Milnor $K$-theory (see $₫ 2$ for more on this).

It turns out that this description generalizes without any problem to the $n$ dimensional case of the pairing in Equation (8). This gives rise to the higher tame symbol. Its role in higher-dimensional class field theory of schemes (as provided by Parshin Par78, Par84, PF99] and Kato Kat79, Kat83, Kato-Saito KS86]) is analogous to the classical tame symbol. Its reciprocity laws have the same formal shape as reciprocity law 99 for rational $n$-forms in Grothendieck-Serre Duality for coherent sheaves. We explain the higher tame symbol and the generalization of the boundary map construction using $\partial$ in $\$ 3$ below.

For the higher tame symbol, one obtains the same object irrespective of whether one uses Milnor $K$-theory or Quillen $K$-theory. This leads us to a first idea how one might construct a higher Contou-Carrère symbol. Firstly, it should be concerned with higher formal loop groups, as in

$$
L^{r} G(A):=G\left(A\left(\left(T_{1}\right)\right)\left(\left(T_{2}\right)\right) \ldots\left(\left(T_{r}\right)\right)\right),
$$

\footnotetext{
${ }^{9}$ They are customarily also called residue theorems in this setting.
} 
which is just the $r$-fold iterate of the loop construction in Equation (11), and is visibly a good formal model for various (equicharacteristic) higher local fields, see Equation (7).

Idea 1. Replace the open-closed complement in Equation (17) by

$$
\operatorname{Spec} A \underset{\text { closed }}{\hookrightarrow} \operatorname{Spec} A[[T]] \underset{\text { open }}{\longleftrightarrow} \operatorname{Spec} A((T))
$$

and attempt to work with the corresponding boundary map $\partial$, imitating the construction of the higher tame symbol. If $A$ is a field, this should specialize to the previous situation and thus, by construction, this generalized symbol would necessarily degenerate to the tame symbol in the classical situation, analogous to what happened around Equation (12)-13.

Firstly, one should ask whether this recovers the original Contou-Carrère symbol even in the one-dimensional case. This had been suggested by Kapranov-Vasserot [KV07, 4.3.7. (Remark)] and is answered affirmatively in this paper (see Theorem [1.3), and was shown around the same time also by Osipov-Zhu [OZ16].

We pursue Idea 1 in §3. It leads to one possible construction of our ContouCarrère symbol in all dimensions (Definition 3.16 ); probably the quickest 10

1.6. Central extensions. On the other hand, this approach also has a drawback: Going from Equation (4) to Equation (5) we chose a local coordinate. In other words, we were using Cohen's Structure Theorem, telling us that an equicharacteristic discrete valuation field is always isomorphic to a Laurent series field,

$$
\operatorname{Frac} \widehat{\mathcal{O}}_{X, x} \simeq \kappa(x)((t)),
$$

where $\kappa(x)$ is the residue field. This isomorphism is highly non-canonical. However, of course none of our constructions should depend on the choice of such a coordinate 11 Translated to the Contou-Carrère symbol, i.e. to abstract loop group functors

$$
L G(A)=G(A((t))),
$$

this suggests that our constructions should really be invariant under all ring automorphisms of $A((t))$, of which there are many 12 This property indeed holds for the original Contou-Carrère symbol, but note that it is not at all obvious from the complicated formula in Equation (11). This suggests to look for a definition of the Contou-Carrère symbol (as well as its higher analogues) where this invariance is automatic by construction.

Tate in his famous paper Tat68] had asked a related question: Suppose $X / k$ is a curve. He wanted to define the residue of a Laurent series at a closed point $x \in X$. While

$$
f \mathrm{~d} t \mapsto a_{-1} \quad \text { for } \quad f=\sum a_{i} t^{i} \in k((t)) \underset{(*)}{\simeq} \operatorname{Frac} \widehat{\mathcal{O}}_{X, x}
$$

is a clear candidate for a definition, it suffers from the same problem of depending on the isomorphism $(*)$. Instead, he wanted a construction which was a priori

\footnotetext{
${ }^{10}$ Actually, we do something more general: Using Parshin-Beilinson adèles one can run such a construction for arbitrary descending chains of subschemes. The case discussed in this introduction arises as a special case.

${ }^{11}$ Just as class field theory doesn't depend on choosing a coordinate.

${ }^{12}$ And themselves representable as a group ind-scheme.
} 
independent of the choice of a coordinate. This issue can be connected to the Contou-Carrère symbol, since it also encodes the residue: The formula

$$
(1-\varepsilon f, 1-\varepsilon g) \equiv 1-\varepsilon^{2} \underset{t=0}{\operatorname{res}}(g \mathrm{~d} f)
$$

holds for the choice $A:=k[\varepsilon] /\left(\varepsilon^{3}\right), k$ any field and regarding $f, g \in k((t))^{\times}$as a subgroup of $L \mathbb{G}_{m}(k)$, APR04, BBE02. Thus, a coordinate-invariant construction of the classical Contou-Carrère symbol includes such a coordinate-independent approach to the residue. Conversely, our second method for constructing a higher Contou-Carrère symbol goes the reverse direction: We adapt Tate's solution for residues in Tat68, which we shall recall in \$1.7 below, to the Contou-Carrère symbol. Tate also showed the residue theorem using his method for curves. Arbarellode Concini-Kac have used the same idea to set up the tame symbol and prove the corresponding reciprocity law on curves [ADCK89] (and more broadly PR02]). Based on this idea, Anderson-Pablos Romo APR04 and Beilinson-Bloch-Esnault BBE02 had the insight that the same strategy should both make it possible to construct the classical Contou-Carrère symbol coordinate-independently and prove its reciprocity law on suitable relative curves.

Next, let us explain how Tate's solution works since this is also the foundation for our second construction of the higher symbol.

1.7. Tate spaces. Let us briefly recall Tate's idea in modern terms: The ingredients for our local symbols can always be written as an ind-pro limit of finitedimensional $k$-vector spaces, e.g.,

$$
k((t))=\underset{n}{\operatorname{colim} \lim _{m}} T^{-n} k[T] /\left(T^{m}\right) \quad \text { or } \quad \operatorname{Frac} \widehat{\mathcal{O}}_{X, x}=\underset{n}{\operatorname{colim} \underset{m}{\underset{m}{m}}} \pi^{-n} \widehat{\mathcal{O}}_{X, x} / \mathfrak{m}_{X, x}^{m}
$$

(for any uniformizer $\pi$ ). Tate had the ingenious insight that for defining the residue, one only needs to know these objects as ind-pro limits 13 He manages to express the residue as a certain commutator of endomorphisms of these ind-pro objects. Since the ind-pro structure on Frac $\widehat{\mathcal{O}}_{X, x}$ can be given without choosing a coordinate isomorphism (as exhibited above on the right), this solves the problem. The commutator in turn can be understood as coming from a central extension of a suitable Lie algebra, i.e. a Lie 2-cocycle in $H^{2}(\mathfrak{g}, k)$ for a suitable Lie algebra $\mathfrak{g}$.

The papers ADCK89, APR04 now recover the tame symbol by studying the corresponding central extension of groups, i.e. a group 2-cocycle $H^{2}\left(G, k^{\times}\right)$for a suitable group. Neglecting various details, one can visualize this as the Lie correspondence between Lie algebras and Lie groups. This is also seen in Equation (21), where the residue is explicitly recovered in a tangent space (to a functor).

In fact, the Lie algebra $\mathfrak{g}$ can be taken to be the endomorphism Lie algebra, and $G$ to be its group of automorphisms (in each case respecting the ind-pro structure).

A key point of the present paper will be to explain how this approach is compatible with the ideas about $K$-theory boundary maps earlier in the introduction. As Anderson-Pablos Romo APR04 set up both the classical Contou-Carrère symbol as well as the reciprocity law using this method, this is another promising approach to construct a higher Contou-Carrère symbol. A natural idea is to iterate the

\footnotetext{
${ }^{13}$ These have since become known as Tate vector spaces. Alternatively (but equivalently), one can work in the setting of locally linearly compact topological $k$-vector spaces. However, the latter setting is problematic to adapt to the relative situation.
} 
ind-pro limits, corresponding to the iterated loop functor in Equation (19).

$$
\begin{aligned}
& A\left(\left(T_{1}\right)\right)\left(\left(T_{2}\right)\right) \cdots\left(\left(T_{r}\right)\right) \\
& \quad=\underset{n_{1}}{\operatorname{colim} \lim _{m_{1}}} \cdots \underset{n_{r}}{\operatorname{colim} \lim _{m_{r}}} T_{1}^{-n_{1}} \cdots T_{r}^{-n_{r}} k\left[T_{1}, \ldots, T_{r}\right] /\left(T_{1}^{m_{1}}, \ldots, T_{r}^{m_{r}}\right) .
\end{aligned}
$$

In order to treat such objects "by induction" in the number of loops $r$, it is natural to set up a category of ind-pro objects with respect to an arbitrary input category so that iterating this categorical construction corresponds to iterating the loop group construction. These are the so-called Tate categories, Pre11, BGW16c. One then finds that the correct analogue of the group 2-cocycles above are higher group $(r+1)$-cocycles for the automorphism groups of suitable objects in such iterated Tate categories (called $r$-Tate categories) 14

The two-dimensional tame symbol and its reciprocity law were set up by Osipov and Osipov-Zhu Osi05, OZ11. Osipov-Zhu also constructed a two-dimensional Contou-Carrère symbol using this method [OZ16] and showed its reciprocity law on surfaces. They also showed how the residue symbol for 2-forms on surfaces is encoded in their symbol, generalizing Equation (21). In the case of the tame symbol these recover the Parshin reciprocity law from his approach to global class field theory.

Idea 2. Construct a higher Contou-Carrère symbol using a generalized central extension, based on a higher group cocycle of an automorphism group of an object in an iterated Tate category.

This will also work and we pursue this in \$5. In some sense it is more general since it really only relies on the iterated ind-pro structures.

1.8. Our approach through homotopy theory. A central part of this paper is devoted to establishing a clear connection between these two ideas. To this end, we need to work with Quillen $K$-theory as a space (or spectrum) and not just the individual $K$-groups. Let us sketch the main idea.

The boundary maps $\partial$ between $K$-groups which appear in Idea 1 really come from maps between spectra 15 e.g., using the boundary map of the localization sequence on the level of spectra,

$$
\Omega K(F) \stackrel{\partial}{\longrightarrow} K(k)
$$

taking $\pi_{1}$ functorially yields the map in Equation (18). Now truncate the homotopy type of $K(k)$ to its $[0,1]$-type. Since $K_{1}(A)=A^{\times}$(at least for local rings, not in general), and if we for simplicity ignore $\pi_{0}$ (which is a serious oversimplification), $K(k)$ essentially looks like $B \mathbb{G}_{m}$. So, very roughly speaking, one almost has a truncation map

$$
\Omega K(F) \stackrel{\partial}{\longrightarrow} K(k) \cdots \cdots \cdots, B \mathbb{G}_{m} .
$$

The dotted arrow does not quite exist because we ignored $\pi_{0}$. Nonetheless, writing $F$-vector spaces as Tate $k$-vector spaces (i.e. as the aforementioned ind-pro limits),

\footnotetext{
${ }^{14}$ This also works on the Lie algebra level. A Lie algebra $(r+1)$-cocycle gives the higher residue symbols of Grothendieck-Serre coherent duality theory; this is due to Beilinson [Bei80; see also Bra18.

${ }^{15}$ We shall provide background on spectra in 4.2 .1
} 
we obtain a map $K(F) \rightarrow K($ Tate $(k))$. Modulo the issues with the dotted map above, there is a factorization

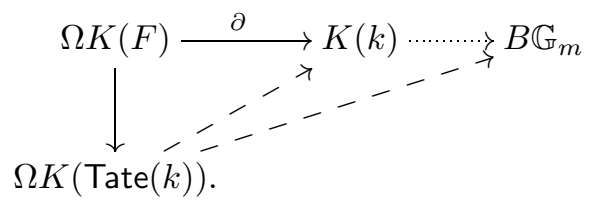

Next, in [BGW18b, Theorem $1.4(2)]$ we showed that

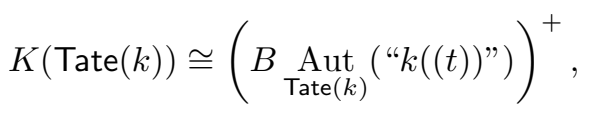

where the plus superscript refers to the plus construction. This is an analogue of Quillen's construction of $K$-theory via the plus construction, i.e., $K(A) \simeq K_{0}(A) \times$ $B \mathrm{GL}(A)^{+}$, except that no corrections to deal with $K_{0}$ are needed and instead of GL we deal with the automorphism group of an object in the Tate category 16 The outer diagonal arrow in Equation (24) thus also pins down a map

$$
\Omega(B \underset{\operatorname{Tate}(k)}{\operatorname{Aut}}(" k((t)) "))^{+} \longrightarrow B \mathbb{G}_{m} .
$$

By adjunction we can move $\Omega$ to the right, lifting $B \mathbb{G}_{m}$ to $B^{2} \mathbb{G}_{m}$. However, one of the defining properties of the plus construction is that it does not affect the homology of a space. Thus, the above map defines a degree 2 cohomology class of the classifying space $B \operatorname{Aut}_{T a t e}(k)(" k((t))$ "), without having applied the plus construction. This is equivalent 17 to providing a group 2-cocycle

$$
H_{g r p}^{2}\left(\underset{\operatorname{Tate}(k)}{\operatorname{Aut}}(" k((t)) "), \mathbb{G}_{m}\right) .
$$

We have explained this in an oversimplified fashion here, especially our imprecise handling of $\pi_{0}$ (which is just wrong). Also, we have not been very precise what categories we work in. Nonetheless, the idea should have become clear. We shall show that a careful variant of the above idea provides the connecting link between defining the Contou-Carrère symbol either via Idea 1 or Idea 2.

The above considerations necessitate to work with $K$-theory on the level of spectra. Moreover, when handling $\pi_{0}$ correctly, the right side in Equation (23) is not just a classifying space, but sits in several homotopical degrees. Thus, one needs to work with a slight generalization of the concept of a group extension when wanting to do this right (we shall work in the context of spectral extensions).

The above picture generalizes to explain also the connection between our two approaches to higher Contou-Carrère symbols. Iterated use of the boundary maps $\partial$ corresponds to an iterated use of Tate categories and a straightforward generalization of Diagram (24). Moreover, all these constructions (including Equation (25), BGW18b, Theorem $1.4(2)])$ work for arbitrary rings $A$ and thus for $A((T))$ and not just $k((t))$, making it possible to use it also in a relative setting.

This leads to our main construction, in the spirit of Idea 2.

\footnotetext{
${ }^{16}$ As we explain in [BGW18b, §4], this result can also be thought of as an algebraic analogue of the Atiyah-Jänich theorem in topological $K$-theory.

${ }^{17}$ The group cohomology group $H_{g r p}^{j}(G, M)$ can equivalently be described as the group of homotopy classes of maps from $B G$ to $B^{j} M$.
} 
Theorem-Construction 1.1. Let $k$ be a field and $A$ a $k$-algebra.

(1) For every $n$-Tate object $\mathcal{V} \in n$-Tate $(A)$, we construct a nontrivial spectral extension of $\operatorname{Aut}(\mathcal{V})$ by the $(n-2)$-shifted non-connective $K$-theory spectrum $\Sigma^{n-2} \mathbb{K}_{A}$ (we leave the detailed construction to the main body of the paper).

(2) Restricting the latter to the units $A\left(\left(t_{1}\right)\right) \cdots\left(\left(t_{n}\right)\right)^{\times}$, they acquire a spectral extension by the non-connective $K$-theory spectrum $\Sigma^{n-2} \mathbb{K}_{A}$. For $f_{0}, \ldots, f_{n}$ $\in A\left(\left(t_{1}\right)\right) \cdots\left(\left(t_{n}\right)\right)^{\times}$we define the Contou-Carrère symbol to be the corresponding higher commutator $\left(f_{0}, \ldots, f_{n}\right)$.

(3) For $n \leq 2$ the constructions of (1) and (2) recover the definitions of ContouCarrère and Osipov-Zhu OZ16].

The first two statements are an immediate consequence of our formalism of spectral extensions and higher commutators (\$4) applied to the $n$-fold iterate of the index map (see Definition [5.4). For the third statement, see Propositions 5.6 and 5.10 .

Denote by $\partial_{i}$ the boundary map in algebraic $K$-theory, where $K(-, I)$ is $K$ theory with support in the subset given by the ideal $I$ :

$$
\partial_{i}: K_{i+1}\left(A\left(\left(t_{1}\right)\right) \cdots\left(\left(t_{i}\right)\right)\left[\left[t_{i+1}\right]\right],\left(t_{i+1}\right)\right) \rightarrow K_{i}\left(A\left(\left(t_{1}\right)\right) \cdots\left(\left(t_{i-1}\right)\right)\left[\left[t_{i}\right]\right],\left(t_{i}\right)\right) .
$$

Let $\pi_{*}: K_{1}\left(A\left[\left[t_{1}\right]\right],\left(t_{1}\right)\right) \rightarrow K_{1}(A)$ be the map induced by $A \rightarrow A\left[\left[t_{1}\right]\right]$, and let $\operatorname{det}: K_{1}(A) \rightarrow A^{\times}$be the determinant.

Theorem 1.2. Let $k$ be a field and $A$ a k-algebra. For $f_{0}, \ldots, f_{n} \in A\left(\left(t_{1}\right)\right) \cdots\left(\left(t_{n}\right)\right)^{\times}$, we have

$$
\left(f_{0}, \ldots, f_{n}\right)^{(-1)^{n}}=\operatorname{det} \pi_{*} \partial_{1} \cdots \partial_{n}\left\{f_{0}, \ldots, f_{n}\right\},
$$

where the left-hand side is our Contou-Carrère symbol of Theorem 1.1. If $A$ is a field, it agrees with the higher tame symbol of Parshin and Kato.

See Theorem 6.5. This theorem shows that Idea 1 is entirely compatible with our construction following Idea 2 .

The right-hand side in the above formula is probably the quickest way to define our higher Contou-Carrère symbol. However, our construction following Idea 2 is more general since Theorem 1.1(1) defines an extension of the entire automorphism group, while the above only sees the restriction to the units of multiplication 18 The key point of the above theorem is that it connects our generalization of the ideas around central extensions as in Arbarello-de Concini-Kac [ADCK89] or AndersonPablos Romo APR04 with the purely algebraic perspective of boundary maps on the right side.

For concreteness, we now state a special case of these results: The following had been conjectured by Kapranov-Vasserot in [KV07.

Theorem 1.3. Let $k$ be a field, and let $A$ be a $k$-algebra. The classical ContouCarrère symbol factors through the boundary map in $K$-theory

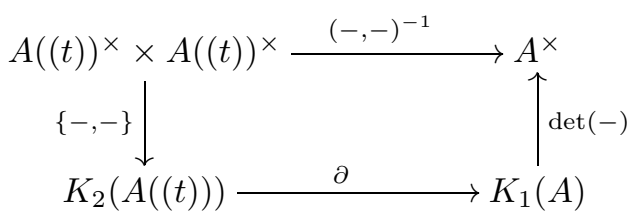

${ }^{18}$ The multiplication with any unit of the ring induces an automorphism of the Tate object. But of course there are many more automorphisms. For example, note that the multiplication automorphisms by units only span a commutative subgroup of the entire automorphism group. 
or, in equations, $(f, g)^{-1}=\operatorname{det}(\partial(\{f, g\}))$. Here $(-,-)$ and $\{-,-\}$ refer to the classical commutator and classical Steinberg symbol respectively.

A second proof of this case has recently appeared in Osipov-Zhu OZ16.

1.9. Grassmannian and determinant bundles. Previous papers on these subjects have constructed the relevant central extensions of $\$ 1.8$, especially the group 2-cocycle in Equation (26), using different devices. The most popular approach to this proceeds by constructing the so-called (regularized) determinant line bundle on the Sato Grassmannian directly. Let us explain this.

Let $k$ be a field as before. Let Pic denote the Picard groupoid of $k$-lines (without grading, for the moment). View $E:=k((t))$ as a Tate $k$-vector space and let $\mathcal{G}$ rass denote its set of lattices 19 Recall that for any finite-dimensional vector space one can define its determinant as its top exterior power

$$
\operatorname{det} V=\bigwedge^{\text {top }} V
$$

and this generalizes nicely to families. Lattices, being infinite-dimensional over $k$, do not a priori have such a determinant. It would not be clear what the "top" exterior power should be once $\operatorname{dim} V=\infty$.

In their approach to the tame symbol cocycle, Arbarello-de Concini-Kac ADCK89] considered maps

$$
\widetilde{\mathrm{det}}: \mathcal{G} \text { rass } \longrightarrow \mathrm{Pic},
$$

associating a line to any lattice. Whenever $L^{\prime} \subseteq L$ for lattices, they demand

$$
\widetilde{\operatorname{det}}(L)=\widetilde{\operatorname{det}}\left(L^{\prime}\right) \otimes \bigwedge^{\text {top }}\left(L / L^{\prime}\right)
$$

to hold, which makes sense since $L / L^{\prime}$ is finite-dimensional over $k$. There are several choices of such maps $\widetilde{\text { det}}$, in fact the set of choices is a $k^{\times}$-torsor. Automorphisms $\operatorname{Aut}(E)$ of $E$ as a Tate vector space do not preserve this choice and rescale the lines. As a result, $\operatorname{Aut}(E)$ does not act on 'the total bundle space' $\coprod_{L \in \mathcal{G} \text { rass }} \widetilde{\operatorname{det}}(L)$, only a central extension does. This central extension yields a class in

$$
H_{g r p}^{2}\left(\operatorname{Aut}(E), k^{\times}\right)
$$

giving the so-called unsigned tame symbol, which is like Equation (13), but without the sign term. This construction can be adapted to $E:=A((t))$, i.e. to the relative situation of $₫ 1.2$. This class is (except for the correct sign), the same one as the one in Equation (26). To get the full theory, Pic can be upgraded to be the Picard groupoid of graded lines $\mathrm{Pic}^{\mathbb{Z}}$. The corresponding cocycle then yields the full classical Contou-Carrère symbol, as was shown by an explicit computation in [APR04, [BBE02].

Essentially, the above is an explicit construction of our homotopical approach in 1.8. It sets up the same cocycle using a group action on the Grassmannian instead of a purely homotopical consideration.

We can also explain our higher Contou-Carrère symbol in terms analogous to the above, an Idea 3 if you will:

(1) The Tate vector space $E$ is generalized to an $n$-Tate object. By the correspondence between 1 -Tate objects of finite-dimensional $k$-vector spaces and locally

\footnotetext{
${ }^{19}$ That lattices are of relevance for our considerations reflects a corresponding phenomenon, where lattices appear in Contou-Carrère's local theory of Jacobians.
} 
linearly compact $k$-vector spaces, this is equivalent to older literature when it refers to similar constructions in terms of linearly compact vector spaces.

(2) The group $\operatorname{Aut}(E)$ is taken to be automorphisms in the category of $n$-Tate objects.

(3) The map det is trickier to generalize. We replace the lattice Grassmannian $\mathcal{G}$ rass by a generalized flag space

$$
L_{0} \hookrightarrow L_{1} \hookrightarrow \cdots \hookrightarrow L_{n} \hookrightarrow E
$$

of nested lattices $L_{i}$ in the $n$-Tate object $E$. We implement an unpublished idea of Kapranov: We generalize det to a map taking values in $K$-theory, without any truncation, and since Waldhausen's explicit $S_{\bullet}$-model for the $K$-theory of $k$ is a simplicial set with simplices $0 \hookrightarrow X_{1} \hookrightarrow \cdots \hookrightarrow X_{n}$, where the $X_{i}$ are finite-dimensional $k$-vector spaces, we may define a map

$$
\left[L_{0} \hookrightarrow L_{1} \hookrightarrow \cdots \hookrightarrow L_{n} \hookrightarrow E\right] \quad \mapsto \quad\left[0 \hookrightarrow L_{1} / L_{0} \hookrightarrow \cdots \hookrightarrow L_{n} / L_{0}\right]
$$

sending flags of lattices to simplices in the $K$-theory space. The special case of just two lattices, $\left[L^{\prime} \hookrightarrow L \hookrightarrow E\right] \mapsto\left[0 \hookrightarrow L / L^{\prime}\right]$, should ring a bell in view of Equation (因). We have worked out the simplicial details of this in our previous paper BGW18b, and use these ideas here.

The role of the Picard groupoids Pic or Pic ${ }^{\mathbb{Z}}$ is seen as follows: Deligne had the insight that there is an equivalence of homotopy categories

$$
\text { stable homotopy }[0,1] \text {-types } \quad \Leftrightarrow \quad \text { Picard groupoids. }
$$

This means that spectra whose homotopy groups vanish outside degrees 0 and 1 can equivalently be modelled by Picard groupoids 20 Thus, our homotopical considerations in $\$ 1.8$ can also be studied using Picard groupoids, at least once we truncate to homotopical degrees 0 and 1 . When one studies the classical ContouCarrère symbol, it is (cum grano salis) almost sufficient to work in such low degrees. Then we use Deligne's insight that $\mathrm{Pic}^{\mathbb{Z}}$ receives a map from the truncated $K$-theory spectrum $\tau_{\leq 1} K$ : The Picard groupoid $\mathrm{Pic}^{\mathbb{Z}}$ is a simplified model for the homotopy type of the 1 -truncation of the Quillen $K$-theory spectrum. This is, by the way, just a different way of expressing how we found $B \mathbb{G}_{m}$ around Equations (22)-(23); Pic is the Picard groupoid corresponding to $B \mathbb{G}_{m}$ under the equivalence in Equation (28).

This is the deeper reason why the above construction can use $\mathrm{Pic}^{\mathbb{Z}}$ and yields equivalent output to what we had otherwise set up in \$1.8 using homotopy types. The need to work with graded lines is the same complication which we had around $\pi_{0}$ in 1.8 .

This discussion also reveals that for higher Contou-Carrère symbols, where higher homotopical degrees are needed, one would have to work in more complicated models than stable $[0,1]$-types.

(4) Cocycles $H^{2}(G, A)$ are classically modelled through commutators. We phrase this as a shuffle product, which generalizes easily to higher degrees. Based on this, we define a concept of higher commutators in 4 . Finally, we interpret all of these constructions consistently through homotopy theory. The group of central extensions $H^{2}(G, A)$ equals the group of homotopy classes of maps (of unpointed spaces) from the classifying space $B G$ to $B^{2} A$. To gain additional flexibility, we

${ }^{20} \mathrm{We}$ shall elaborate a little on this and related facts in 4.2 .1 
define a notion of spectral extension. It amounts to maps $\Sigma_{+}^{\infty} B G$ to $\Sigma^{2} \mathbb{E}$ for a spectrum $\mathbb{E}$ (where $\Sigma_{+}^{\infty} X$ denotes the infinite suspension of a (unpointed) space $X$ with a disjoint basepoint added). This turns out to be the appropriate language to generalize the Contou-Carrère symbol. See $\$ 4$ for details.

Main Principle. Ideas 1, 2 and 3 all yield the same concept of a higher ContouCarrère symbol.

The compatibility of Idea 1 and Idea 2 is Theorem 1.2 and the compatibility to Idea 3 is part of Theorem 1.1 (3).

1.10. Higher reciprocity laws. Our next result is a type of adelic reciprocity law: let $X$ be a reduced, separated $k$-scheme of finite type and dimension $n$. Fix an integer $0 \leq i \leq n$. Let $\zeta$ denote a flag of integral closed subschemes

$$
\zeta=\left(Z_{n} \supset Z_{n-1} \supset \cdots \supset Z_{i+1} \supset Z_{i-1} \supset \cdots \supset Z_{0}\right),
$$

indexed by $j \neq i$, with $\operatorname{dim} Z_{j}=j$. If $i=0$, we assume that $Z_{1}$ is proper over $k$. Exactly one dimension is missing, namely $Z_{i}$; such flags are called almost saturated. We denote by $A_{X, \zeta}$ a certain ring formed as an iterated completion of $A(X)$, the $A$-valued rational functions on $X$, at the places $Z_{j} \times{ }_{k} \operatorname{Spec}(A)$, cf. 33.1 . As for the classical adèles, the ring $A_{X, \zeta}$ is built from rings $A_{X, \zeta_{Z}}$, one for each $i$-dimensional closed subset

$$
Z_{i-1} \subset Z \subset Z_{i+1}
$$

Each of these rings carries a higher Contou-Carrère symbol $\left(f_{0}, \ldots, f_{n}\right)_{\xi_{Z}}$, and the geometry of $A_{X, \zeta}$ gives rise to a relation satisfied by these symbols:

Theorem 1.4. For $f_{0}, \ldots, f_{n} \in A_{X, \zeta}^{\times}$the product of the Contou-Carrère symbols over all $Z_{i-1} \subset Z \subset Z_{i+1}$ is well defined, and we have

$$
\prod_{Z}\left(f_{0}, \ldots, f_{n}\right)_{\zeta_{Z}}=1
$$

See Theorem [7.4. This theorem extends results for $X$ of dimension one by Anderson-Pablos Romo [APR04 and Pál Pál10] (for A 0-dimensional), BeilinsonBloch-Esnault [BBE02] (for $A$ arbitrary), and results for $X$ of dimension 2 by Osipov-Zhu OZ16.

The finite dimensionality of the cohomology of a proper curve provides a key geometric input in proving the reciprocity law for 1-dimensional symbols. In the setting of higher dimensional reciprocity laws, we can morally interpret the ring $A_{X, \zeta}$ of Theorem 1.4 as the ring of $A$-valued rational functions of an exotic "curve" $X_{\zeta}$ associated to the almost saturated flag $\left\{Z_{j}\right\}_{j \neq i}$. In principle, this "curve" should be obtained by iteratively completing $X$ at the $Z_{j}$ and then removing the special point $Z_{j}$. However, at present, the theories of Berkovich or rigid analytic spaces are insufficient to handle such constructions. Rather than develop such a theory, we take a non-commutative geometry approach and replace $X$ by its stable $\infty$-category of perfect complexes. The operations of localization and completion of schemes have analogues for stable $\infty$-categories, cf. Thomason-Trobaugh [TT90] (localization) and Efimov [Efi10] (completion). We apply these in $\$ 7$ to construct a stable $\infty$ category which plays the role of "Perf $\left(X_{\zeta}\right)$ " and we use the (non-commutative) "geometry" of this stable $\infty$-category to deduce the reciprocity law. 
These categorical constructions could be pictured as a "non-commutative shadow" of the formal scheme obtained by formal completion. Their role should be understood to be analogous to the one of the "commutative shadows" utilized by Contou-Carrère (and called ombres in [CC94, [CC13]).

For our proof of reciprocity, we adopt a general strategy which was first introduced by Gillet Gil78 21 The reciprocity law of Theorem 1.4 expresses information about the local geometry of a variety around an almost saturated flag. As remarked above, our approach to higher symbols allows us to reduce the reciprocity law to the statement that $d^{2}=0$ in a Gersten-style complex. As with the classical Gersten complex, the differentials arise as (sums of) boundary maps in $K$-theory localization sequences. Our work on derived completion allows us to obtain these localization sequences in our setting and deduce reciprocity.

We now explain the strategy of this proof in the case $A=k$ and $n=2$. Let $Y$ be a smooth surface over $k$ and $x \in Y$ a closed point. For a triple of non-zero elements $f, g, h$ of the fraction field of $\mathcal{O}_{Y, y}$ we must show that the product

$$
\prod_{C}(f, g, h)_{C, x}
$$

ranging over curves containing $x$, is well-defined and equals 1 . There exists a closed subset $Z \subset Y$, such that $Z$ is a union of curves containing $x$, and $f, g, h$ are regular elements on $U=Y \backslash Z$. Our results above identify this product with a composition of boundary maps as in the lower path of the diagram

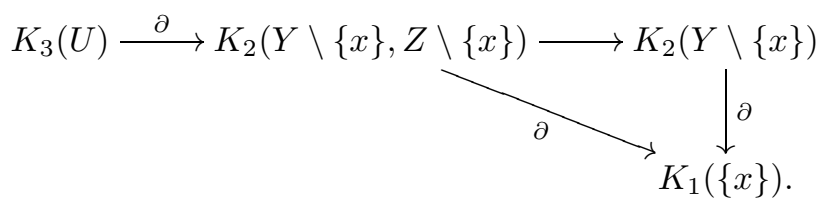

However, this is also equivalent to the upper path of the diagram, the last two maps of which are successive maps in a long exact sequence.

For dimension $n>2$, we employ an analogous argument. However, we must now replace the punctured surface $\operatorname{Spec} \mathcal{O}_{Y, x}-\{x\}$ with a more exotic object obtained by completing and removing at all the closed subsets in an almost saturated flag. Our treatment of derived completions supplies us with the necessary localization sequences in this setting, while our treatment of symbols allows us to identify the appropriate product with a composition of boundary maps from these sequences. It is then a relatively straightforward matter to show that this composition is zero when restricted to tuples of invertible elements of $A_{X, \zeta}$.

\section{K-THEORY}

2.1. Background on the flavours of $K$-theory. We shall use $K$-theory in various flavours, so let us quickly recall the key players and motivate how and why they enter our considerations.

\footnotetext{
${ }^{21}$ We thank the first anonymous referee for bringing this to our attention.
} 
2.1.1. Origins. Historically inspired by the study of vector bundles in algebraic geometry, one can form for any (small) exact category $\mathrm{C}$ the $K_{0}$-group

$$
K_{0}(\mathrm{C})=\frac{\{\text { iso-classes }[X] \text { of objects } X \in \mathrm{C}\}}{\text { relations }[X]=\left[X^{\prime}\right]+\left[X^{\prime \prime}\right] \text { for any exact sequence } X^{\prime} \hookrightarrow X \rightarrow X^{\prime \prime}} .
$$

Choosing $\mathrm{C}$ to be the exact category of vector bundles $\mathrm{VB}(X)$ on a variety $X$, this provided the necessary context for Grothendieck's extension of the classical Riemann-Roch theorem.

The freedom to develop the whole theory for very general categories instead of just vector bundles has proven very useful and will also be vital for our considerations.

2.1.2. Localization (geometry). Returning to vector bundles, studying the relationship of the $K_{0}$-group for a scheme $X$ in comparison to the one of a reduced closed subscheme $Z \subseteq X$ and its open complement $U=X-Z$ leads to "higher" $K$-groups fitting together into the so-called localization sequence. In this geometric setting (and only if everything is smooth), it takes the form of an exact sequence

$$
\cdots \longrightarrow K_{n}(Z) \longrightarrow K_{n}(X) \longrightarrow K_{n}(U) \longrightarrow K_{n-1}(Z) \longrightarrow \cdots .
$$

In fact, this long exact sequence can be understood in terms of different categories. For example, still assuming everything to be smooth, one gets the relevant $K_{0^{-}}$ groups by taking the category of coherent sheaves on $Z, X$ and $U$ respectively, and obtains

$$
\operatorname{Coh}(U)=\operatorname{Coh}(X) / \operatorname{Coh}_{Z}(X),
$$

expressing the category of coherent sheaves on the open complement $U$ as the quotient abelian category of the coherent sheaves on $X$, modulo those having support in $Z$, called $\operatorname{Coh}_{Z}(X)$. In other words: The decomposition of $X$ into $Z$ and its complement $U$ can be reflected as a subcategory and the respective quotient on the level of categories. This suggests a general picture for categories, valid beyond this geometric application:

2.1.3. Localization (general principles). Generalized to arbitrary (say abelian or exact categories) $\mathrm{C}$ and suitable subcategories $\mathrm{C}^{\prime} \subseteq \mathrm{C}$, the above picture generalizes to long exact sequences

$$
\cdots \longrightarrow K_{n}\left(\mathrm{C}^{\prime}\right) \longrightarrow K_{n}(\mathrm{C}) \longrightarrow K_{n}\left(\mathrm{C} / \mathrm{C}^{\prime}\right) \longrightarrow K_{n-1}\left(\mathrm{C}^{\prime}\right) \longrightarrow \cdots
$$

In the hands of Quillen, general algebraic $K$-theory was defined as the homotopy groups of certain spaces attached to (for example) exact categories, as in

$$
K_{n}(\mathrm{C}):=\pi_{n} K(\mathrm{C}),
$$

where $K(\mathrm{C})$ is a pointed space. There are several ways to set up $K(\mathrm{C})$; e.g., as a simplicial set using simplicial homotopy theory or as a topological spaces using classical homotopy theory. Moreover, there are different ways to set up these spaces, all leading to the same homotopy type (e.g., the $Q$ - or $S$-construction). These differences are not so important for the present paper. Background for simplicial homotopy theory can be found for example in May92, Lam68, or GJ09. 
2.1.4. Finer points. To get a really nice picture, the above suggests various improvements:

(1a) As the $K$-groups are defined as the homotopy groups of a space as in Equation (31), it is natural to hope that the long exact sequences in Equation (29) resp. Equation (30) stem from fiber sequences of pointed spaces. This can indeed be implemented and leads to defining Quillen $K$-theory as an invariant of certain categories, taking values in pointed spaces. This path is already taken by Quillen Qui73 or Waldhausen Wal85. These two approaches only differ in generality, but yield the same theory, which in this paper will be called connective $K$-theory.

(1b) Actually, the pointed spaces $K(\mathrm{C})$ of connective $K$-theory are of a very special type: They come equipped with the structure of an infinite loop space Ada78. While infinite loop spaces can be regarded on the one hand as pointed spaces with extra structure, they can equivalently be regarded as connective spectra, i.e. spectra $S$ such that $\pi_{i} S=0$ for all $i<0$. Thus, modulo switching between equivalent categories, the $K(-)$ in Equation (31) can alternatively be taken to refer to a (connective) spectrum. Background on spectra can be found for example in [Wei94, §10.9] (for a survey), or in [HSS00, § 1], Hov01, § 1] or [Lura, §1.4]for more general treatments.

(2) The sequence in Equation (29) only exists under very restrictive assumptions, and using Quillen's $K$-theory it is not right-exact at $K_{0}$. However, this nuisance can be smoothened out and leads to slightly modified versions of $K$-theory. Nowadays, and also in the present paper, these are all jointly generalized to the so-called nonconnective $K$-theory (we recall the details below). A general construction on the level of arbitrary exact categories is given in Sch06. The cited paper also proves the compatibility with the previous approaches to resolve this issue (e.g., the socalled Bass 'negative $K$-groups' Bas68] or Thomason-Trobaugh $K$-theory [TT90]). Unfortunately, there is no way to fix the lack of exactness at $K_{0}$ without needing negative $K$-groups further to the right in the respective sequences as in Equation (130). Thus, non-connective $K$-theory cannot really be modelled in spaces. However, the property to be a spectrum remains intact also for non-connective $K$-theory. Hence, the natural habitat for non-connective $K$-theory are spectra. This time, however, they are not necessarily connective. In particular, it is not necessarily possible to still model this using infinite loop spaces instead of spectra.

Remark 2.1. This also explains the names of connective and non-connective $K$ theory. This use of terminology is also in line with the conventions of [BGT13, which shows that one can also describe the two variants of $K$-theory in terms of certain universal properties, giving a further justification to work with both theories in parallel, yet carefully distinguish between them.

(3) Quillen's foundations for connective $K$-theory from Qui73 allow all exact categories as input; and similarly Sch06] gives similar foundations for nonconnective $K$-theory. However, wanting a very general localization sequence as in Equation (30) there is an issue with the formation of the quotient $C / C^{\prime}$. For many natural choices of exact categories and subcategories this quotient does not reasonably exist as an exact category. Going beyond this, there are various interesting categories, for example arising from glueing constructions of categories, which are of a profoundly more subtle nature than what can be captured through the formalism of exact categories. To this end, it roughly speaking makes sense to generalize $K$-theory to accept all stable $\infty$-categories [Lura] as input. Abelian 
categories (or exact categories) have a natural attached stable $\infty$-category, so that this is a genuine generalization. This generalization is available for both connective and non-connective $K$-theory, and as described above, BGT13. describes either in terms of a universal property whose formulation also necessitates the use of stable $\infty$-categories.

2.1.5. Milnor K-theory. Finally, we shall also use Milnor $K$-theory. Classically, this is only defined for fields, even though the definition can be extended to local rings Ker09, Ker10]. At least for fields $F$, one just has

$$
K_{n}^{M}(F):=\frac{T_{\mathbb{Z}}^{*}\left(F^{\times}\right)}{\langle x \otimes(1-x) \text { for all } x \in F \backslash\{0,1\}\rangle},
$$

where $T_{\mathbb{Z}}^{*}(M):=\bigoplus_{n>0} M^{\otimes_{\mathbb{Z}}^{n}}$ denotes the free tensor algebra of an abelian group. Historically, this was regarded as a candidate definition for higher $K$-groups, but since then the picture has clarified a lot: In this paper we mostly refer to Milnor $K$-theory because of the simplicity of its definition, or the natural graded ring homomorphism

$$
K_{*}^{M}(F) \longrightarrow K_{*}(F),
$$

which easily exhibits high degree elements in the connective $K$-theory of fields.

Remark 2.2. The deeper truth however is that the motivic Atiyah-Hirzebruch spectral sequence starts from motivic cohomology $H^{n}(F, \mathbb{Z}(m))$ on the $E_{2}$-page and converges to connective $K$-theory. It satisfies

$$
H^{n}(F, \mathbb{Z}(n)) \cong K_{n}^{M}(F),
$$

so the deeper reason for the similarities between Milnor and connective $K$-theory (of a field) is just their 'proximity' as provided by the motivic weight filtration on the $K$-theory spectrum, exhibited here through the spectral sequence. The comparison of this with étale K-theory (resp. étale motivic cohomology) also lies at the core behind the compatibility to Galois cohomology in Diagram (15). However, none of this is needed in the present paper. See MVW06 and Gei05] for background.

2.2. Axiomatic review of algebraic $K$-theory. After this review, let us summarize the key statements we shall need in the format most suitable for us. We view algebraic $K$-theory as a machine, which assigns, to an exact category or stable $\infty$-category $C$, its spectral shadow $\mathbb{K}_{C}$. This machine sends exact functors $C \rightarrow D$ to maps of spectra $\mathbb{K}_{C} \rightarrow \mathbb{K}_{\mathrm{D}}$, and preserves exact sequences. We refer the reader to appendix $\mathrm{A}$ for a brief overview of the theory of (stable and unstable) $\infty$-categories, and to Lur09b, Lura] for detailed references.

We encourage the reader unfamiliar with stable $\infty$-categories to think of them as a higher homotopical enrichment of triangulated categories. For example, by Lura, Theorem 1.1.2.14], the homotopy category $\mathrm{Ho}(\mathrm{C})$ of any stable $\infty$-category inherits a canonical triangulated structure. The advantage of working with stable $\infty$-categories is that many standard constructions for triangulated categories become better behaved and more conceptually straightforward in this context.

Recall that given an $\infty$-category C, we can form an $\infty$-category of "ind-objects" $\operatorname{lnd}(\mathrm{C})$ (with subcategories $\operatorname{Ind}_{\kappa}(\mathrm{C})$ for each regular $\kappa$ ) of "formal filtered colimits" of diagrams in $C$ (or such over diagrams of size at most $\kappa$ ) (see Lur09b, §5.3.5]). If $C$ is a stable $\infty$-category, then so is $\operatorname{Ind}_{\kappa}(\mathrm{C})$ Lura, Prop. 1.1.3.6]. Similarly, every $\infty$-category $C$ admits an idempotent completion $C \rightarrow C^{\text {ic }}$ (see [Lur09b, §5.1.4]), and 
if $C$ is stable, so is $C^{\text {ic }}$ Lura, Cor. 1.1.3.7]. Last, just as there is a good notion of exact functors of triangulated categories $C \rightarrow D$ and of exact sequences

$$
\mathrm{C} \rightarrow \mathrm{D} \rightarrow \mathrm{D} / \mathrm{C}
$$

of such functors, there is a good notion of such for stable $\infty$-categories. In fact, by [BGT13, Prop. 5.1.5], a sequence of stable $\infty$-categories is exact if and only if the induced sequence of homotopy categories is an exact sequence of triangulated categories. In particular, given a fully faithful exact functor of (presentable) stable $\infty$-categories $C \rightarrow D$, we can form the quotient stable $\infty$-category $D / C$, which we should think of as a higher homotopical analogue of the classical Verdier quotient.

2.2.1. Connective algebraic $K$-theory. The proposition below captures the most important phenomena for the so-called connective $K$-theory of stable $\infty$-categories (cf. BGT13). This is the flavour of $K$-theory which is compatible with Quillen's original definition of algebraic $K$-theory.

In the following we denote by $\mathrm{Sp}_{>}$the stable $\infty$-category of connective spectra. We refer the reader to Subsection 4.2.1 for a brief reminder of stable homotopy theory, and for more details to Wei94, §10.9] (for a survey), [HSS00, §1], [Hov01, § 1] or [Lura, §1.4].

Proposition 2.3. The functor of connective $K$-theory for stable $\infty$-categories

$$
\mathcal{K}_{-}: \mathrm{Cat}_{\infty, s t} \rightarrow \mathrm{Sp}_{\geq}
$$

satisfies the following properties.

(1) If $\mathrm{C}$ is a stable $\infty$-category admitting countable products (or coproducts), then $\mathcal{K}_{\mathrm{C}} \cong 0$.

(2) The inclusion $\mathrm{C} \rightarrow \mathrm{C}^{\mathrm{ic}}$ (where ic denotes idempotent completion) gives rise to a map of connective spectra $\mathcal{K}_{\mathrm{C}} \rightarrow \mathcal{K}_{\mathrm{C}^{\mathrm{ic}}}$, inducing an isomorphism on $\pi_{i}$ for $i \geq 1$, and a monomorphism on $\pi_{0}$.

(3) Let $\mathrm{C} \hookrightarrow \mathrm{D} \rightarrow \mathrm{D} / \mathrm{C}$ be an exact sequence of stable $\infty$-categories, where we denote the functor $\mathrm{C} \rightarrow \mathrm{D}$ by $i$ and $\mathrm{D} \rightarrow \mathrm{D} / \mathrm{C}$ by $q$. Then, there is a fibre sequence

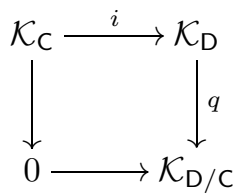

in the $\infty$-category $\mathrm{Sp}_{\geq}$of connective spectra.

Property (3) is often referred to as proto-localization (e.g. by [TT90]). The long exact fibration sequence for $\pi_{*}$ yields a long exact sequence in non-negative degrees. The map $\pi_{0}\left(\mathcal{K}_{\mathrm{D}}\right) \rightarrow \pi_{0}\left(\mathcal{K}_{\mathrm{D} / \mathrm{C}}\right)$ will not be surjective in general 22 This suggests the existence of negative $K$-groups, obtained by the homotopy groups of a non-connective $K$-theory spectrum. This leads to non-connective $K$-theory, whose properties we recall in the following section.

\footnotetext{
${ }^{22}$ This is the same issue which already appears in purely geometric applications and is alluded to in 2.1 .4
} 
2.2.2. Non-connective algebraic K-theory. In the work of Blumberg-GepnerTabuada, the following properties were shown to be characteristic for non-connective $K$-theory (see [BGT13, Thm. 9.10]). In the following we denote by Sp the stable $\infty$-category of all spectra.

Proposition 2.4. Non-connective algebraic $K$-theory is a functor

$$
\mathbb{K}_{-}: \mathrm{Cat}_{\infty, s t} \rightarrow \mathrm{Sp}
$$

satisfying the following properties.

(1) If $\mathrm{C}$ is a stable $\infty$-category admitting countable products (or countable coproducts), then $\mathbb{K}_{\mathrm{C}} \cong 0$.

(2) The inclusion $\mathrm{C} \rightarrow \mathrm{C}^{\mathrm{ic}}$ (where ic denotes idempotent completion) gives rise to an equivalence of spectra $\mathbb{K}_{\mathrm{C}} \stackrel{\cong}{\rightarrow} \mathbb{K}_{\mathrm{C}}$.

(3) Let $\mathrm{C} \hookrightarrow \mathrm{D} \rightarrow \mathrm{D} / \mathrm{C}$ be an exact sequence of stable $\infty$-categories, where we denote the functor $\mathrm{C} \rightarrow \mathrm{D}$ by $i$ and $\mathrm{D} \rightarrow \mathrm{D} / \mathrm{C}$ by $q$. Then, there is a a bi-cartesian square

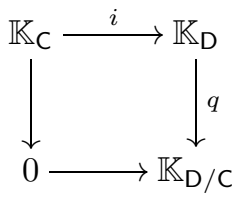

in the stable $\infty$-category $\mathrm{Sp}$ of spectra.

We say that non-connective $K$-theory $\mathbb{K}_{-}$completes connective $K$-theory $\mathcal{K}_{-}$, referring to the canonical equivalence

$$
\mathcal{K}_{\mathrm{C}^{\text {ic }}} \cong \tau_{\geq 0} \mathbb{K}_{\mathrm{C}} .
$$

Following Schlichting [Sch06], we see how every connective theory, satisfying the axioms of Proposition 2.3, induces a non-connective $K$-theory, subject to the properties of Proposition 2.4 (see also [BGT13]). This requires the suspension of a stable $\infty$-category.

Definition 2.5. We define the suspension of a stable $\infty$-category $C$ as the stable $\infty$-category

$$
\mathcal{S}_{\kappa}(\mathrm{C})=\operatorname{lnd}_{\kappa} \mathrm{C} / \mathrm{C}
$$

where $\kappa$ denotes an arbitrary infinite cardinal, and $\operatorname{Ind}_{\kappa} \mathrm{C}$ denotes the stable $\infty$ category of Ind-objects represented by diagrams of size at most $\kappa$. Let Calk $_{\kappa}(\mathrm{C})$ denote $\mathcal{S}_{\kappa}(\mathrm{C})^{\text {ic }}$.

By definition, we have an exact sequence of stable $\infty$-categories

$$
\mathrm{C} \hookrightarrow \operatorname{lnd}(\mathrm{C}) \rightarrow \mathcal{S}(\mathrm{C}) .
$$

Using the fact that Ind (C) admits countable coproducts, properties (1) and (3) of Proposition 2.3 imply that

$$
\mathcal{K}_{\mathrm{C}} \rightarrow 0 \rightarrow \mathcal{K}_{\mathcal{S}(\mathrm{C})}
$$

is a fibre sequence of connective spectra. Since $\pi_{0}(0)=\pi_{0}(\mathcal{S}(\mathrm{C}))$, we know that it is actually a fibre-cofibre sequence of spectra. This allows us to identify $\mathcal{K}_{C}$ with $\Omega \mathcal{K}_{\mathcal{S}(\mathrm{C})}$. We define the non-connective completion $\mathbb{K}_{-}$to be the functor

$$
\underset{\longrightarrow}{\longrightarrow} \Omega^{n} \mathcal{K}_{\text {Calk }^{n}(\mathrm{C})}
$$

where Calk $^{0}(\mathrm{C}):=\mathrm{C}$ and Calk $^{n}(\mathrm{C}):=$ Calk $\left(\right.$ Calk $\left.^{n-1}(\mathrm{C})\right)$ for $n>0$. 
Definition 2.6. Let $C$ be an (idempotent complete) exact category. We have a well-defined dg-category $\mathrm{Ch}^{b}(\mathrm{C})$ of bounded chain complexes in $\mathrm{C}$. We denote by $\mathrm{Ch}_{a c}^{b}(\mathrm{C})$ the full subcategory of acyclic complexes. The stable $\infty$-category Perf(C) is defined to be the dg-nerve (see Lura, §1.3.1]) of the dg-quotient $\mathrm{Ch}^{b}(\mathrm{C}) / \mathrm{Ch}_{a c}^{b}(\mathrm{C})$. Since the latter is a pre-triangulated dg-category (see [Kel99, §2]), Perf(C) is stable.

The lemma below follows from the discussion in [BGT13, §9.1] and Sch06, §6.2]

Lemma 2.7. Let $\mathrm{C}$ be an exact category. The non-connective $K$-theory of $\mathrm{C}$, in the sense of Schlichting [Sch06], agrees with the non-connective K-theory of the stable $\infty$-category Perf(C) in the sense of Blumberg-Gepner-Tabuada BGT13.

It will be necessary to compare algebraic $K$-theory with the original category, in order to be able to use it. Heuristically, this is captured by the slogan that $\mathbb{K}_{C}$ is a spectrum, where objects in $\mathrm{C}$ give rise to points, automorphisms of objects give rise to loops, and, for $n \geq 1$, commuting $n$-tuples of automorphisms in $\mathrm{C}$ give rise to elements of $K_{n}(\mathrm{C})=\pi_{n}\left(\mathbb{K}_{\mathrm{C}}\right)$. This intuition is captured by the following observation.

Remark 2.8. We denote by $\mathrm{C}^{\times}$the $(\infty-)$ groupoid of objects in $\mathrm{C}$ (i.e. we discard all non-isomorphisms). Recall that every $(\infty-)$ groupoid can be viewed as an unpointed space via the geometric realization of its nerve 23 There exists a canonical morphism of pointed spaces $\left(\mathrm{C}^{\times}\right)_{+} \rightarrow \Omega^{\infty} \mathbb{K}_{\mathrm{C}}$, and by the adjunction $\Sigma^{\infty} \dashv \Omega^{\infty}$, a morphism of spectra $\Sigma_{+}^{\infty} \mathrm{C}^{\times} \rightarrow \mathbb{K}_{\mathrm{C}}$, see [Wal85, $\S 1.3$, p. 12].

Example 2.9. Under special circumstances the last map of the previous remark can be promoted to an equivalence. Such a phenomenon underlies Equation (25) along with the fact that the Tate category has vanishing $K_{0}$-group.

Definition 2.10. We shall frequently use the following shorthands:

(1) If $R$ is a ring, we write $\mathbb{K}_{R}$ to denote the nonconnective K-theory spectrum of the category of perfect complexes over $R$.

(2) Analogously, if $X$ denotes a scheme, we write $\mathbb{K}_{X}$ for the nonconnective K-theory spectrum of perfect complexes on $X$.

(3) If $X$ has a closed subscheme $Z$, we write $\mathbb{K}_{X, Z}$ for the nonconnective $\mathrm{K}$ theory spectrum of the category of perfect complexes on $X$ with support in $Z$. The latter means that they are required to be acyclic over the complement $X-Z$.

Example 2.11. If $X$ is Noetherian (for example) and has the closed subscheme $Z$, then there is an exact sequence relating their stable $\infty$-categories of perfect complexes. Using Proposition 2.4 (3) we obtain the fibre sequence

$$
\mathbb{K}_{X, Z} \rightarrow \mathbb{K}_{X} \rightarrow \mathbb{K}_{X-Z}
$$

of spectra. The induced long exact sequence of the homotopy groups of the $K$ theory spectra is perhaps the most prominent example of the localization sequence.

${ }^{23} \mathrm{We}$ review the nerve, i.e. the ways of regarding a category as a simplicial set or space in A.1.2 


\section{The CC Symbol via boundary maps}

In this section we will give a first definition of our higher Contou-Carrère symbol. We follow a generalization of the idea of boundary maps (which we had called Idea 1 in the introduction). Instead of working with iterated loop groups, we use localizations-completions along flags of subschemes. Abstractly, these look like iterated loop groups, but our methods avoids choosing a coordinate. The case discussed in the introduction follows as a special case (see Example 3.1).

3.1. Flags of closed subschemes. Let $X$ be a reduced excellent separated scheme of dimension $n$. A flag is a sequence

$$
\xi: Z_{n} \supset \cdots \supset Z_{0}
$$

of integral closed subschemes of pure dimension $\operatorname{dim} Z_{i}=i$, indexed by a subset of $\{0,1, \ldots, n\}$. If it is indexed by all of $\{0,1, \ldots, n\}$, we call it saturated. If exactly one dimension is missing, such flags are called almost saturated.

Definition 3.1. If $\xi:=\left(Z_{n} \supset \cdots \supset Z_{0}\right)$ denotes a flag, we abbreviate the ParshinBeilinson adèle ring by

$$
\left.F_{X, \xi}:=A\left(\xi, \mathcal{O}_{X}\right)=A\left(\left\{\left(Z_{n} \supset \cdots \supset Z_{0}\right)\right\}\right), \mathcal{O}_{X}\right) .
$$

The notation $A(-,-)$ is as in Beilinson's original paper [Bei80, §2].

The definition of these adèles is alternatively also given in Hub91, Proposition 2.1.1] or [BGW16a, §2.1], viewed from different angles.

Example 3.2. This section also covers the case relevant for

$$
L^{n} G(A):=G\left(A\left(\left(T_{1}\right)\right)\left(\left(T_{2}\right)\right) \ldots\left(\left(T_{n}\right)\right)\right)
$$

as discussed in the introduction. Choose $X$ to be affine $n$-space and take a standard flag of coordinate hyperplane subspaces. See also Example 7.6.

For a saturated flag, there is a canonical isomorphism of rings

$$
F_{X, \xi} \cong \prod_{i=1}^{r} F_{i}
$$

for some finite $r$, and each $F_{i}$ is an $n$-local field. For a proof, see [Yek92, §3] or BGW16a, Theorem 4.2].

Whenever $F$ denotes an $n$-local field, this means that it comes with a canonically determined diagram

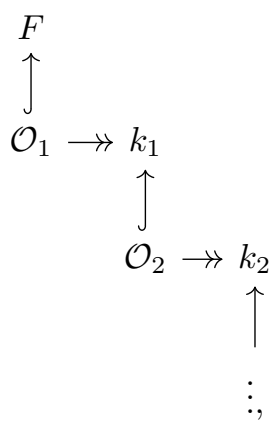

where each $\mathcal{O}_{i}$ denotes the rings of integers of the field depicted above it, and each $k_{i}$ denotes the residue field of the ring depicted to its left. 
The $K$-groups of the various rings attached to $F$ are related by the localization sequence

$$
\cdots \rightarrow K_{i}(k) \rightarrow K_{i}(\mathcal{O}) \rightarrow K_{i}(F) \stackrel{\partial}{\rightarrow} K_{i-1}(k) \rightarrow \cdots,
$$

which can be used inductively for each step in the above downward ladder of residue fields because of an identification of $\mathbb{K}_{\mathcal{O}, \mathfrak{m}}$ with the $K$-theory of the residue field $\kappa(\mathfrak{m}) 24$

The above localization sequence stems from the bi-cartesian square

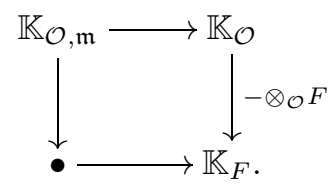

In order to obtain this square, apply Example 2.11 to $\operatorname{Spec} \mathcal{O}$ and the closed subscheme cut out by the unique maximal ideal (the valuation ideal). The open complement is just $\operatorname{Spec} F$. In the present situation it makes no difference whether we use connective or non-connective $K$-theory.

Remark 3.3. We will soon generalize the above by instead using the square in Equation (37) below.

Definition 3.4. Let $F$ be an $n$-local field.

(1) The higher tame symbol (in Quillen $K$-theory) is defined to be the composition

$$
\partial^{(1)} \circ \cdots \circ \partial^{(n)}: K_{n+1}(F) \rightarrow K_{1}(\kappa)=\kappa^{\times},
$$

where $\kappa$ is the last residue field and $\partial$ refers to the respective boundary maps coming (inductively for each residue field) from the localization sequence in (36).

(2) In many ways simpler, the higher tame symbol (in Milnor K-theory) is defined to be the composition

$$
\partial^{(1)} \circ \cdots \circ \partial^{(n)}: K_{n+1}^{M}(F) \rightarrow K_{1}(\kappa)=\kappa^{\times},
$$

where $K^{M}$ refers to Milnor $K$-theory and $\partial$ is the boundary map in an entirely analogous localization sequence in motivic cohomology. See Remark 2.2 for the relation between Quillen and Milnor $K$-theory.

The higher tame symbol in Milnor $K$-theory is simpler because Milnor $K$-groups have a generator-relator presentation and the relevant boundary map can alternatively be defined by an explicit formula. See [Mil70, §2]. In fact, historically this was known before the interpretation as motivic cohomology. Only the latter however shows how closely connected both viewpoints are.

We move on to the relative situation.

Definition 3.5. If $X$ is additionally a scheme of finite type over $k$, then for every $k$-algebra $A$ we define

$$
A_{X, \xi}:=F_{X, \xi} \otimes_{k} A,
$$

and for saturated flags, we note that the canonical isomorphism of Equation (34) can be promoted to an isomorphism of $k$-algebras, see [BGW16a, Theorem 4.2].

\footnotetext{
${ }^{24}$ As the rings are all regular, we can also work with the $K$-theory of coherent sheaves, where devissage applies. Hence, the $K$-theory of coherent sheaves with support in the maximal ideal is equivalent to the $K$-theory of the residue field. This yields the identification.
} 
Following Morrow's [Mor, we give a self-contained construction of $F_{X, \xi}$, in a format which will be particularly useful for us later.

Definition 3.6. An ideal $I \subset R$ of a Noetherian $\operatorname{ring} R$ is called equiheighted if all minimal prime ideals over $I$ have the same height in $R$. We define the localization of an $R$-module $M$ at $I$, to be

$$
M_{I}=S^{-1} M, \text { where } S=\{s \in R \mid s \text { is a non-zero-divisor in } R / I\} .
$$

Geometrically, an equiheighted ideal defines a closed subspace of Spec $R$, with all irreducible components having the same codimension in Spec $R$. Although not completely obvious, the two operations introduced below preserve chains of equiheighted ideals [Mor, Lemma 7.3].

Definition 3.7. Let $R$ be a Noetherian ring of Krull dimension $n$. For a chain of equiheighted ideals $\xi=\left(I_{k} \subset \cdots \subset I_{0}\right)$, with ht $I_{i}=n-i$, we define the completion operation

We denote by

$$
\mathrm{C}(R, \xi)=\left(\widehat{R}_{I_{0}}, \xi\right)
$$

$$
\mathrm{L}(R, \xi)=\left(R_{I_{1}}, \xi^{\prime}\right),
$$

the localization operation, where $\xi^{\prime}$ is the restriction to $R_{I_{1}}$ of the shifted chain of ideals given by $I_{i}^{\prime}=I_{i+1}$.

Example 3.8. If $R$ is a Noetherian domain of Krull dimension 1, then for every prime ideal $\mathfrak{p}$, we can consider the chain $\xi=(0 \subset \mathfrak{p})$. In this case, we have $(\mathrm{L} \circ \mathrm{C})(R, \xi)=\operatorname{Frac} \widehat{R}_{\mathfrak{p}}$.

Definition 3.9. Let $R$ be an excellent reduced ring of Krull dimension $n$. For a chain of radical equiheighted ideas $\left(0=I_{n} \subset I_{n-1} \subset \cdots \subset I_{0}\right)$, with ht $I_{i}=n-i$, we define

$$
F_{\text {Spec } R, \xi} \cong(\mathrm{L} \circ \mathrm{C})^{n}(R, \xi) .
$$

This definition is compatible with Definition 3.1.

3.2. Boundary maps of a flag. We begin by giving a precise definition of the completion of a scheme at a closed subscheme. Although this seems fairly straightforward in the affine case, it is necessary to be finical in general.

Definition 3.10. Let $X$ be a scheme and $\mathcal{I} \subset \mathcal{O}_{X}$ a sheaf of ideals for which the corresponding closed subscheme $Y \subset X$ is affine. We define the completion of $X$ at $Y$ to be the affine scheme

$$
\mathbf{C}_{Y} X=\operatorname{Spec} \lim _{n \in \mathbb{N}} \Gamma\left(X, \mathcal{O}_{X} / \mathcal{I}^{n}\right)
$$

A related construction is the formal neighbourhood $\widehat{X}_{Y}$. It is defined to be the direct limit in the sense of formal schemes, of the family of schemes $Y_{n}=$ $\operatorname{Spec}_{X} \mathcal{O}_{X} / \mathcal{I}^{n}$. The completion of Definition 3.10 on the other hand is equivalent to the direct limit of the $X$-schemes $Y_{n}$ in the category of affine schemes.

Warning 3.11. The definition above could lead to pathological situations if $Y$ was not assumed to be affine. For example, if $Y \subset \mathbb{P}^{n}$ is an embedded projective curve, the inverse limit $\lim _{n \in \mathbb{N}} \mathcal{O}_{X} / \mathcal{I}^{n}$ in the category of $\mathcal{O}_{X}$-modules is not necessarily quasi-coherent. 
By virtue of Chevalley's theorem Gro64 (or for a recent exposition, see Con$\operatorname{rad}$ [Con07]), affineness of $Y$ only depends on the underlying reduced subscheme $Y^{\text {red }} \subset X$ (i.e. Chevalley's theorem implies that a scheme is affine if and only if the associated reduced scheme is affine).

Given a variety with a flag of closed subschemes one can iteratively complete and localize at the flag. This is captured by the following algorithmic definition.

Definition 3.12. Let $X$ be a Noetherian $k$-scheme and $A$ a $k$-algebra. Given a flag of closed subschemes $\xi: X=Z_{n} \supset Z_{n-1} \supset \cdots \supset Z_{0} \supset Z_{-1}=\emptyset$, with $Z_{i}$ of pure dimension $i$, we define a collection of schemes $X^{(i)}$ for $i=-1, \ldots, n-1$ by running the following recursive algorithm:

(a) $X^{(-1)}=X_{A}=X \times_{k} \operatorname{Spec} A$,

(b) $Z_{j}^{(k)}=X_{j}^{(k)} \times_{X} Z_{j}$,

(c) $X^{(k)}=\mathbf{C}_{Z_{k}^{(k-1)}}\left(X^{(k-1)}\right) \backslash Z_{k}^{(k-1)}$.

We need to verify that this algorithm is well-defined, by checking that the affineness condition of Definition 3.10 is satisfied whenever we perform step (c). This is the content of the following lemma.

Lemma 3.13. For $k \geq 0$ the schemes $Z_{k}^{(k-1)}$ are affine.

Proof. This statement is established by induction on $k$, where the base case $k=0$ is clear, since $X^{(0)}=\mathbf{C}_{Z_{0}^{-1}} X^{(-1)}$ is defined to be the completion of $X^{(-1)}=$ $X \times_{k}$ Spec $A$ at the affine scheme $Z_{0} \times_{k} A$. Here we used that $Z_{0}$ was assumed to be zero-dimensional, and therefore is automatically affine.

Let us assume that the assertion is known for all $k \geq 0$, which satisfy $k \leq m$. We will show that it also holds for $k=m+1$. By definition we have

$$
Z_{m+1}^{(m)}=X^{(m)} \times_{X} Z_{m+1} .
$$

Since $Z_{m+1} \hookrightarrow X$ is a closed immersion (hence in particular affine), we see that the projection map $Z_{m+1}^{(m)}=X^{(m)} \times_{X} Z_{m+1} \rightarrow X^{(m)}$ is also a closed immersion (and therefore affine). Moreover, the scheme $X^{(m)}$ is constructed as the completion at the scheme $Z_{m}^{(m-1)}$, which we know to be affine by the induction hypothesis. This shows that $X^{(m)}$ is affine, and therefore that the closed subscheme $Z_{m+1}^{(m)}$ is affine too. This concludes the proof.

Remark 3.14. Pullback along the natural morphism

$$
\left(\mathbf{C}_{Z_{m+1}^{(m)}} X^{(m)}, Z_{m+1}^{(m)}\right) \rightarrow\left(X^{(m)}, Z_{m+1}^{(m)}\right)
$$

of pairs induces an equivalence of derived categories of perfect complexes with support condition. In particular we have an equivalence of $K$-theory $\mathbb{K}_{\mathbf{C}_{Z_{m+1}^{(m)}} X^{(m)}, Z_{m+1}^{(m)}}$ $\simeq \mathbb{K}_{X^{(m)}, Z_{m+1}^{(m)}}$. For $A$ a Noetherian ring this is a direct consequence of Theorem 2.6.3 in TT90]. The proof of the general case is deferred to Proposition B.8 in the appendix.

We recall the following result from Thomason-Trobaugh [TT90, Porism 2.7.1].

Lemma 3.15. Let $X$ be a scheme of finite type over $k$, with a subscheme $Z$ finite over $k$ (in particular $\operatorname{dim} Z=0$ ). For every $k$-algebra $A$, we denote by

$$
\pi: X_{A}=X \times_{k} \operatorname{Spec} A \rightarrow \operatorname{Spec} A
$$


the canonical projection. If $\mathcal{F} \in \operatorname{Perf}_{Z_{A}}\left(X_{A}\right)$, then $\pi_{*} \mathcal{F}$ is a perfect complex of A-modules.

Proof. This is a special case of Porism 2.7.1 in [TT90. Up to change of notation, the latter considers a finitely presented map $h: X \rightarrow W$, a quasi-compact open subset $U \subset X$, which is the complement of a closed immersion $Z \rightarrow X$, such that $\left.h\right|_{Z}$ is proper, and $\left.h\right|_{U}$ is flat. Under these assumptions it is shown that the pushforward $h_{*} \mathcal{F}$ of a perfect complex $\mathcal{F}$ supported on $|Z|$, is perfect.

In order to apply this result, one observes that a morphism of finite type over a field is finitely presented. Moreover, being of finite presentation, flat, or proper, is a notion invariant under base change. Since every finite morphism is in particular proper, all the conditions of the porism cited above are met.

We are now in a position to state the main result of this section. At first we need to introduce some notation. We denote by $\partial_{m}$ the morphism of $K$-theory spectra

$$
\partial_{m}: \Omega^{m} \mathbb{K}_{X^{(m)}, Z_{m+1}^{(m)}} \rightarrow \Omega^{m-1} \mathbb{K}_{X^{(m-1)}, Z_{m}^{(m-1)}},
$$

obtained as the boundary map of the bi-cartesian square

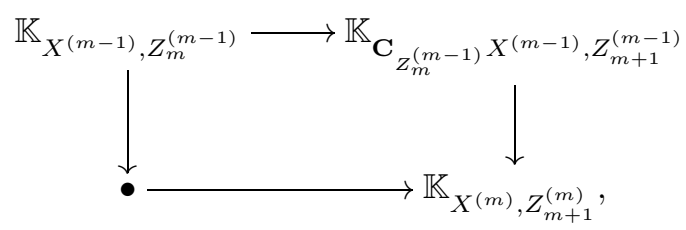

where we have used the equivalence $\mathbb{K}_{\mathbf{C}_{Z_{m}^{(m-1)}} X^{(m-1)}, Z_{m}^{(m-1)}} \simeq \mathbb{K}_{X^{(m-1)}, Z_{m}^{(m-1)}}$ of Remark 3.14. The morphisms in the bi-cartesian square above are induced by the inclusion maps between the respective pairs of schemes.

Definition 3.16 (Preliminary Contou-Carrère symbol). Let $X$ be a Noetherian $k$-scheme, and $\xi$ a saturated flag of closed subschemes $Z_{i}$. For every $k$-algebra $A$, we have a projection $\pi: X_{A} \rightarrow \operatorname{Spec} A$. The pushforward $\pi_{*}$ sends $\operatorname{Perf}_{Z_{A}}\left(X_{A}\right)$ to $\operatorname{Perf}(A)$. Hence, we have a well-defined map

$$
\pi_{*} \circ \partial_{1} \circ \cdots \circ \partial_{n}: \Omega^{n} \mathbb{K}_{A_{X, \xi}} \rightarrow \mathbb{K}_{A} .
$$

We call this the preliminary Contou-Carrère symbol $\sigma_{X, \xi}^{A}$.

\section{Spectral extensions And higher COMmutators}

In this section we introduce the notion of a central extension of a group by a spectrum. We then define a generalization of the commutator pairing for such spectral extensions and relate it to Loday's Steinberg symbols in algebraic $K$-theory.

\subsection{Classical central extensions.}

4.1.1. Central extensions. Let $A$ be an abelian group. A central extension of $G$ by $A$, denoted $e$, is a short exact sequence

$$
1 \rightarrow A \rightarrow^{\iota} E \rightarrow^{p} G \rightarrow 1,
$$

such that $\iota(A) \subset Z(E)$, where $Z(E) \subset E$ denotes the centre of $E$.

Definition 4.1. We denote by $P_{n}(G)$ the set of $n$-tuples of pairwise commuting elements

$$
\left\{\left(g_{1}, \ldots, g_{n}\right) \in G^{n} \mid g_{i} g_{j}=g_{j} g_{i} \forall 1 \leq i, j \leq n\right\} .
$$


Given $(f, g) \in P_{2}(G)$, let $\tilde{f}, \widetilde{g}$ be elements in $p^{-1}(f)$, respectively $p^{-1}(g)$. Since $p\left(\widetilde{f} \widetilde{g} \tilde{f}^{-1} \widetilde{g}^{-1}\right)=1$, we see that the commutator $[\widetilde{f}, \widetilde{g}]=\widetilde{f} \widetilde{g} \widetilde{f}^{-1} \widetilde{g}^{-1}$ defines an element in $A$. Because $A$ is central in $E$, a simple computation shows that this element is independent of the choice of liftings.

Definition 4.2. Let $e$ be a central extension of $G$ by $A$. We denote by $\star_{e}: P_{2}(G) \rightarrow$ $A$ the function $(f, g) \mapsto \iota^{-1}[\tilde{f}, \widetilde{g}]$.

Short calculations Bro82, Exercise IV.3.8(a)] show that $\star$ is bi-multiplicative and anti-symmetric. For the convenience of the reader we include a proof.

Lemma 4.3. For $\left(g_{1}, g_{2}, h\right) \in P_{3}(G)$, we have the following relations:

(a) $\left(g_{1} \star_{e} h\right) \cdot\left(g_{2} \star_{e} h\right)=g_{1} g_{2} \star_{e} h$,

(b) $\left(g_{1} \star_{e} g_{2}\right)^{-1}=g_{2} \star_{e} g_{1}$.

Proof. The first identity can be established by the following computation:

$$
\begin{aligned}
\left(g_{1} \star_{e} h\right) \cdot\left(g_{2} \star_{e} h\right) & =\left(\widetilde{g}_{1} \widetilde{h} \widetilde{g}_{1}^{-1} \widetilde{h}^{-1}\right) \cdot\left(\widetilde{g} \widetilde{h}^{-1} \widetilde{g}_{2}^{-1}\right)=\widetilde{g}_{1}\left(\widetilde{g}_{2} \widetilde{h} \widetilde{g}_{2}^{-1} \widetilde{h}^{-1}\right) \widetilde{h} \widetilde{g}_{1}^{-1} \widetilde{h}^{-1} \\
& =\widetilde{g}_{1} \widetilde{g}_{2} \widetilde{h} \widetilde{g}_{2}^{-1} \widetilde{g}_{1}^{-1} \widetilde{h}^{-1}=\left(g_{1} g_{2}\right) \star_{e} h,
\end{aligned}
$$

where in the second equality sign we used that $\left(\widetilde{g}_{2} \widetilde{h}_{2}^{-1} \widetilde{h}^{-1}\right)$ belongs to the centre of $G$. The second identity follows from

$$
\left(g_{1} \star_{e} \widetilde{g}_{2}\right)^{-1}=\left(\widetilde{g}_{1} \widetilde{g}_{2} \widetilde{g}_{1}^{-1} \widetilde{g}_{2}^{-1}\right)^{-1}=\widetilde{g}_{2} \widetilde{g}_{1} \widetilde{g}_{2}^{-1} \widetilde{g}_{1}^{-1}=g_{2} \star_{e} g_{1} .
$$

This concludes the proof of the lemma.

A central extension $e$ as in (38) corresponds to a monoidal map from $G$ to the groupoid $B A$ (that is, the groupoid of $A$-torsors with the natural symmetric monoidal structure). To see this directly, one observes that every fibre $p^{-1}(g) \subset$ $E$ has the structure of an $A$-torsor. Moreover, we have a natural isomorphism $p^{-1}(g h) \cong p^{-1}(g) \otimes_{A} p^{-1}(h)$ for every pair $(g, h) \in G^{2}$. Thus, (38) gives rise to a map of monoidal groupoids

$$
\phi: G \rightarrow B A,
$$

where $G$ is viewed as a discrete groupoid with monoidal structure given by the group operation, and $B A$ denotes the classifying groupoid of $A$-torsors. The following interpretation of the commutator pairing is well-known.

Lemma 4.4. For $(f, g) \in P_{2}(G)$ we have that $f \star g$ corresponds to the automorphism in $B A$ obtained from the following chain of morphisms

$$
\phi(f g) \cong \phi(f) \phi(g) \cong \phi(g) \phi(f) \cong \phi(g f)=\phi(f g) .
$$

Proof. Choosing lifts $\tilde{f}$ of $f$ and $\widetilde{g}$ of $g$, we can express the torsors $\phi(f)$ as $A \cdot \tilde{f}$ and $\phi(g)$ as $A \cdot \widetilde{g}$. We can also write

$$
\phi(f g) \cong A \cdot \widetilde{f} \widetilde{g} \cong \phi(f) \otimes_{A} \phi(g) .
$$

The symmetry constraint of $\otimes_{A}$ induces an isomorphism with $A \cdot \widetilde{g} \tilde{f}$, which sends $\widetilde{f} \widetilde{g}$ to $[\widetilde{f}, \widetilde{g}] \widetilde{g} \widetilde{f}$.

Using the identification $\phi(g) \phi(f) \cong \phi(g f)=\phi(f g)$ the element $\widetilde{g} \widetilde{f}$ is sent to $\widetilde{f} \widetilde{g}$. We conclude that the resulting automorphism of the torsor $\phi(f g) \cong A \cdot \widetilde{f} \widetilde{g}$ sends the element $\widetilde{f} \widetilde{g}$ to $[\tilde{f}, \widetilde{g}] \widetilde{f} \widetilde{g}$. Therefore, it corresponds to the commutator pairing $f \star g$. 
4.1.2. Cohomological reformulation. The map (39) is the looping of a map of pointed spaces

$$
e:(B G, *) \rightarrow\left(B^{2} A, *\right) .
$$

Since the target is equivalent to an Eilenberg-Mac Lane space $B^{2} A \cong K(A, 2)$ (as unpointed spaces), homotopy classes of (unpointed) maps $B G \rightarrow B^{2} A$ agree with $H^{2}(B G, A)=H_{g r p}^{2}(G, A)$. We denote the element in this cohomology group resulting from $e$ by $[e]$.

If $G$ is an abelian group, then the group homology $H_{*}(B G, \mathbb{Z})=H_{*}^{g r p}(G, \mathbb{Z})$ carries a natural graded commutative ring structure. Topologically this follows from $B G$ inheriting a group structure from the commutative group $G$, endowing it with the structure of an $H$-group. Algebraically, this fact can be explained in terms of the shuffle product on the normalized bar complex. In the remark below we recall its definition.

Remark 4.5. Recall that the $\mathbb{Z} G$-module $B_{k}$ is defined to be the free module on symbols $\left(g_{1}|\ldots| g_{k}\right)$, where the $g_{i}$ are pairwise distinct elements of the group $G$. Using that $G$ is abelian, we define

$$
\left(g_{1}|\ldots| g_{k}\right) \circ\left(g_{k+1}|\ldots| g_{k+l}\right)=\sum_{\sigma}(-1)^{\sigma}\left(g_{\sigma^{-1}(1)}|\ldots| g_{\sigma^{-1}(k+l)}\right),
$$

where $\sigma$ runs over all permutations of $\{1, \ldots, k+l\}$ satisfying $\sigma(1) \leq \cdots \leq \sigma(k)$ and $\sigma(k+1) \leq \cdots \leq \sigma(k+l)$ (so-called shuffles). Extending $\mathbb{Z} G$-linearly, the shuffle product endows $\bigoplus_{k} B_{k}$ with the structure of a commutative dg-algebra.

This graded commutative ring structure brings us to the following definition.

Definition 4.6. Let $G$ be an arbitrary group. Given $\left(g_{1}, \ldots, g_{n}\right) \in P_{n}(G)$, we denote by $\phi: \mathbb{Z}^{n} \rightarrow G$ the corresponding morphism of groups, sending the standard vector $e_{i}$ to $g_{i}$. Let $c$ denote $\left(e_{1} \circ \cdots \circ e_{n}\right)$ as in Remark 4.5. We set $\left(g_{1} \circ \cdots \circ g_{n}\right):=$ $\phi_{*}(c)$.

The class in $H_{2}^{g r p}(G, \mathbb{Z})$ corresponding to the cycle $(f \circ g)$ should be understood as an abstract commutator. A pair $(f, g)$ of commuting elements induces a map $\mathbb{T}^{2}=$ $B \mathbb{Z}^{2} \rightarrow B G$. Topologically speaking, the cycle $(f \circ g)$ is obtained by pushforward of the fundamental class of the torus $B \mathbb{Z}^{2}$ to $B G$.

The following lemma is standard (e.g. it is an immediate consequence of Bro82, Exercise IV.3.8.(b,c)] combined with [Bro82, Theorem V.6.4(iii)]).

Lemma 4.7. Let $\langle-,-\rangle$ denote the natural pairing between group cohomology and homology. Given a central extension e of $G$ by $A$, corresponding to the class $[e] \in$ $H_{g r p}^{2}(G, A)$, we have for all $(f, g) \in P_{2}(G)$ the identity

$$
f \star_{e} g=\langle[e],(f \circ g)\rangle .
$$

The following definition illustrates the flexibility of the cohomological viewpoint on commutators. We use the notation $K(A, k)$ to denote an unpointed space, which represents the (co-)functor $H^{k}(-, A)$ valued in abelian groups.

Definition 4.8. A higher central extension of $G$ by $B^{k} A=K(A, k)$ is an element $[e]$ of $H_{g r p}^{k+2}(G, A)$. Given $\left(g_{1}, \ldots, g_{k+2}\right) \in P_{k+2}(G)$ we define

$$
g_{1} \star_{e} \cdots \star_{e} g_{k+2}=\left\langle[e],\left(g_{1} \circ \cdots \circ g_{k+2}\right)\right\rangle \text {. }
$$

In the following subsection we will formally generalize this definition to include central extensions by arbitrary spectra, not just those of Eilenberg-Mac Lane type. 


\subsection{Spectral extensions.}

4.2.1. Stable $\infty$-categories and spectra. A fundamental example of a stable $\infty$ category (see A.2) is given by the stable $\infty$-category $\mathrm{Sp}$ of spectra, which is defined to be the limit

$$
\mathrm{Sp}:=\lim _{\longleftarrow}\left[\text { Space }_{\bullet} \stackrel{\Omega}{\leftarrow} \text { Space }_{\bullet} \stackrel{\Omega}{\leftarrow} \cdots\right] .
$$

where Space $_{*}$ denotes the category of pointed spaces, and $\Omega$ denotes the pointed loop space functor.

Every pointed space $\mathcal{X}=\left(X, x_{0}\right)$ gives rise to a spectrum, denoted by $\Sigma^{\infty} \mathcal{X}$. The infinite suspension functor $\Sigma^{\infty}$ has a right adjoint

$$
\Sigma^{\infty}: \text { Space. } \rightarrow \text { Sp, namely } \quad \Omega^{\infty}: \mathrm{Sp} \rightarrow \text { Space. }_{\bullet}
$$

The latter functor is equivalent to the projection to the first component

$$
\lim _{\longleftarrow}\left[\text { Space. } \stackrel{\Omega}{\leftarrow} \text { Space }_{\bullet} \stackrel{\Omega}{\leftarrow} \cdots\right] \rightarrow \text { Space. } .
$$

There is an array of functors to the category of abelian groups $\left(\pi_{i}\right)_{i \in \mathbb{Z}}: \mathrm{Sp} \rightarrow \mathrm{Ab}$, inducing a $t$-structure on Sp with heart $\mathrm{Sp}^{\mathcal{O}}=\left\{X \in \mathrm{Sp} \mid \pi_{i}(X)=0\right.$ for $\left.i \neq 0\right\} \cong \mathrm{Ab}$.

The subcategory $\mathrm{Sp}_{[0,1]}=\left\{X \in \mathrm{Sp} \mid \pi_{i}(X)=0\right.$ for $\left.i \neq 0,1\right\}$ is equivalent to the 2-category of Picard groupoids (that is, group-like symmetric monoidal groupoids). More generally, the $\infty$-category of connective spectra $\mathrm{Sp}_{>}=\left\{X \in \mathrm{Sp} \mid \pi_{i}(X)=\right.$ 0 for $i \leq-1\}$ is equivalent to the $\infty$-category of Segal's $\Gamma$-spaces (i.e. Picard $\infty$ groupoids, or equivalently, infinite loop spaces).

The behaviour of the $\infty$-category of spectra with respect to this $t$-structure reveals a remarkable similarity with the derived category $D(\mathbb{Z})$ of abelian groups. This time we have homology groups

$$
H_{i}: D(\mathbb{Z}) \rightarrow \mathrm{Ab},
$$

inducing a $t$-structure on $D(\mathbb{Z})$. Again, the heart $D(\mathbb{Z})^{\ominus}$ is equivalent to the category of abelian groups. Chain complexes in $D(\mathbb{Z})_{[0,1]}$, i.e. those concentrated in degree 0 and 1 , are, according to a theorem of Deligne, equivalent to strictly commutative Picard groupoids. The Dold-Kan correspondence asserts that objects in $D(\mathbb{Z})_{\geq 0}$ correspond to simplicial abelian groups.

It seems therefore appropriate to think of spectra as another generalization of abelian groups. The derived category of abelian groups serves a similar purpose, but working with spectra corresponds to only stipulating a weak commutativity law, which allows spectra to capture phenomena which could not be seen in the strict framework of chain complexes of abelian groups.

4.2.2. Generalized group cohomology. For every spectrum $\mathbb{E}$, we have an associated generalized cohomology theory denoted by

$$
H^{i}(-, \mathbb{E}): \text { Space } \rightarrow \text { Ab. }
$$

We define generalized group cohomology to be $H_{\text {grp }}^{i}(G, \mathbb{E})=H^{i}(B G, \mathbb{E})$.

Definition 4.9. A spectral extension of $G$ by $\mathbb{E}$ is a class $[e] \in H_{g r p}^{2}(G, \mathbb{E})$.

Every abelian group $A$ can be viewed as a spectrum $H A$ by means of the Eilenberg-Mac Lane construction. Forgetting base points, we have an equivalence of unpointed spaces $\Omega^{\infty} \Sigma^{k} H A \simeq B^{k} A$.

A higher central extension of $G$ by $B^{k} A$ in the sense of Definition 4.8, is given by an element of $H_{g r p}^{k+2}(G, A)=H_{g r p}^{2}\left(G, \Sigma^{k} H A\right)$. By the discussion above, we can 
therefore say that a higher central extension of $G$ by $B^{k} A$ is the same thing as a spectral extension of $G$ by the $k$-fold suspension spectrum $\Sigma^{k} H A$.

Remark 4.10. The definition of a spectral extension given in Definition 4.9 introduces only the cocycle (up to equivalence) of what should be a central extension by a spectrum. Without doubt it would be possible to give a definition along the lines of Paragraph 4.1.1. However, spelling out such a definition would certainly be more cumbersome than the shortcut used in Definition 4.9, which is exactly the viewpoint we need to study higher commutators in the next paragraph.

\subsection{The case of spectral extensions.}

4.3.1. Basic definitions. Our definition of higher commutators for spectral extensions hinges on three $\infty$-categories whose objects belong to the canon of classical homotopy theory. These $\infty$-categories have already made an appearance earlier in the paper.

(a) The $\infty$-category of unpointed spaces Space, as defined in Lurb, Definition 1.2.16.1].

(b) The $\infty$-category of pointed spaces will be referred to as Space. (see Lura, Notation 1.4.2.5]).

(c) The stable $\infty$-category of spectra Sp (see [Lura, Definition 1.4.3.1]).

All three $\infty$-categories happen to be generated under small colimits by a single object. Spaces Space are generated by the singleton $\{\bullet\}$ (see Lurb, Theorem 5.1.5.6] applied to $S$ being the $\infty$-category consisting of a single object and only the identity morphism), pointed spaces Space. by a pointed space with two elements $\left(S^{0}, x_{0}\right)$ (combine the aforementioned result, and Lura, Proposition 4.8.2.11]), and Sp is generated by the sphere spectrum $\mathbb{S}$ (see [Lura, Corollary 1.4.4.6]). Furthermore, these $\infty$-categories do not just exist in isolation from each other, but are related by a chain of functors.

(d) The functor $(-)_{+}$: Space $\rightarrow$ Space. is well-defined (up to a contractible space of choices) by the fact that it commutes with small colimits and sends the singleton space $\{\bullet\}$ to a pointed space with two elements $\left(S^{0}, x_{0}\right)$. Informally speaking it assigns to a space $X$ the pointed space $X_{+}$obtained as the disjoint union $X \sqcup\left\{x_{0}\right\}$ with base point $x_{0}$.

(e) The infinite suspension functor $\Sigma^{\infty}$ : Space. $\rightarrow$ Sp is well-defined (up to a contractible space of choices) by stipulating that it commutes with small colimits, and sends $\left(S^{0}, x_{0}\right)$ to the sphere spectrum $\mathbb{S}$.

Definition 4.11. The composition of $(-)_{+}$and $\Sigma^{\infty}$ will be denoted by $\Sigma_{+}^{\infty}$.

The fact that Space, Space. and Sp are generated by one object is not only convenient for defining functors between them, but also implies directly that they are presentable $\infty$-categories (see [urb, Theorem 5.5.1.1(6)]). In Lura, §4.8.2], a symmetric monoidal structure on $\operatorname{Pr}^{L}$ (the $\infty$-category of small presentable $\infty$ categories) is used to establish the existence of symmetric monoidal smash products on Space. and Sp, as well as compatibility between them. Just as one can talk of commutative algebra objects in a symmetric monoidal (1-)category, one can talk about analogous objects in a symmetric monoidal $\infty$-category. These go by the name of $E_{\infty}$-rings or $E_{\infty}$-objects (see [May77 or [Lura, $\left.\S 7\right]$ ). We encourage the reader to think of these as a higher homotopical analogue of commutative rings, or commutative DGAs. 
4.3.2. Short summary. The functor $\Sigma_{+}^{\infty}$ : Space $\rightarrow$ Sp is symmetric monoidal with respect to the cartesian symmetric monoidal structure on unpointed spaces and the smash product of spectra $\otimes$. That is, for two unpointed spaces $X, Y$ we have $\Sigma_{+}^{\infty}(X \times Y) \simeq \Sigma_{+}^{\infty} X \otimes \Sigma_{+}^{\infty} Y$.

In particular this functor preserves $E_{\infty}$-objects. We conclude that $\Sigma_{+}^{\infty} B \mathbb{Z}$ is a commutative ring spectrum. This induces a graded commutative product structure on $\pi_{*}$ and allows one to define higher commutators. We will now describe all of this in more detail.

\subsubsection{Facts from modern homotopy theory.}

(f) There exists a canonical symmetric monoidal structure on Space, the cartesian symmetric monoidal structure (see Lura, Sect. 2.4.3]). We denote the corresponding symmetric monoidal $\infty$-category by Space $_{x}$. Up to a contractible space of choices it is well-defined by the fact that $\{\bullet\}$ is a unit, and the induced bi-functor $\times$ : Space $\times$ Space $\rightarrow$ Space commutes in both variables with small colimits.

(g) There exists a canonical symmetric monoidal structure on Space, , we denote the symmetric monoidal category by $\left(\text { Space. }_{\bullet}\right)_{\wedge}$. Up to a contractible space of choices it is well-defined by the property of having $\left(S^{0}, x_{0}\right)$ as a

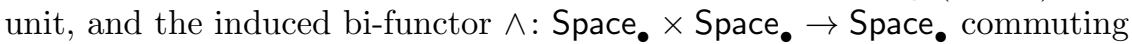
with small colimits in both variables (see [Lura, Remark 4.8.2.11]).

(h) There exists a canonical symmetric monoidal structure on $\mathrm{Sp}$, we denote the symmetric monoidal category by $(S p)_{\otimes}$. Up to a contractible space of choices it is well-defined by the property of having the sphere spectrum $\mathbb{S}$ as a unit, and the induced bi-functor $\otimes: \mathrm{Sp} \times \mathrm{Sp} \rightarrow \mathrm{Sp}$ commuting with small colimits in both variables (see Lura, Corollary 4.8.2.19]).

(i) The functors $(-)_{+}$and $\Sigma^{\infty}$ have a natural symmetric monoidal structure (well-defined up to a contractible space of choices). In particular we have equivalences (well-defined up to a contractible space)

$$
X_{+} \wedge Y_{+} \simeq(X \times Y)_{+}
$$

for $X, Y \in$ Space and

$$
\Sigma^{\infty} \mathcal{X} \otimes \Sigma^{\infty} \mathcal{Y} \simeq \Sigma^{\infty}(\mathcal{X} \wedge \mathcal{Y})
$$

for $\mathcal{X}, \mathcal{Y} \in$ Space.

The facts (f)-(i) are well-known outside of the context of stable $\infty$-categories. After passing to homotopy categories, one recovers the classical concepts. In particular, the symmetric monoidal structure $\wedge$ on Space. may be thought of as the smash product of pointed spaces

$$
\left(X, x_{0}\right) \wedge\left(Y, y_{0}\right)=\left((X \times Y) /\left(X \times\left\{y_{0}\right\} \cup\left\{x_{0}\right\} \times Y\right),\left(x_{0}, y_{0}\right)\right) .
$$

The advantage of the present approach is that it foregrounds the treatment of homotopy coherence, rather than having to build this after the fact for a particular space-level construction. We give a more detailed account of the proof of (i), since it is only implicit in Lura, $§ 4.8 .2$ ].

Proof of (i). In Lura, Definition 4.8.2.8] Lurie defines what it means for a small colimit preserving functor of presentable $\infty$-categories Space $\rightarrow C$ to realize $C$ as an idempotent object. It is then shown (see Lura, Proposition 4.8.2.9]) that there is an equivalence on the full subcategory of $\operatorname{Fun}\left(\Delta^{1}, \operatorname{Pr}^{L}\right)$ corresponding to such 
morphisms, and the over-category of presentable symmetric monoidal $\infty$-categories over Space . .

In [Lura, Proposition 4.8.2.11 \& 4.8.2.18] it is shown that Space. and Sp are naturally idempotent categories with respect to the canonical functors Space $\rightarrow^{(-)_{+}}$ Space. and Space $\rightarrow^{\Sigma_{+}^{\infty}}$ Sp. This is then used to deduce the existence of the symmetric monoidal structure mentioned in $(\mathrm{g}),(\mathrm{h})$. The same observation implies that $(-)_{+}$and $\Sigma^{\infty}$ are symmetric monoidal functors (see Lura, Proposition 4.8.2.7]).

Putting all of the facts recited above together, we obtain the following consequences.

Corollary 4.12. The composition of functors $\Sigma^{\infty}(-)_{+}$oforget: Space $\rightarrow$ Space $\rightarrow$ Sp is naturally equivalent to $\mathbb{S} \oplus \Sigma^{\infty}(-)$.

Proof. We begin by considering the category of pointed simplicial sets, which we will use as a model for the $\infty$-category of pointed spaces. Let $\mathcal{X}=\left(X, x_{0}\right)$ be a pointed simplicial set. We use the notation $S^{0}$ to denote the pointed simplicial set corresponding to the 0 -sphere. We denote by $i: S^{0} \rightarrow X_{+}$the map of pointed simplicial sets which sends the base point of $S^{0}$ to the base point of $X_{+}$, and the unique non-base point of $S^{0}$ to $x_{0} \in X$. Let $s: X_{+} \rightarrow S^{0}$ be the unique left-inverse to this map in the category of pointed simplicial sets. We denote by $g: X_{+} \rightarrow \mathcal{X}$ the unique map of pointed simplicial sets, such that $\left.g\right|_{X}=\mathrm{id}{ }_{X}$. These maps belong to a natural commutative diagram of pointed simplicial sets

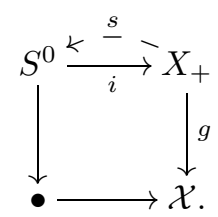

The dashed arrow refers to the well-defined retract in pointed simplicial sets. For every $X$ there is a unique retract, hence it is natural. Passing from model categories to $\infty$-categories (see [Lurb, A.2(2)] we obtain a natural commutative diagram of functors taking values in Space.

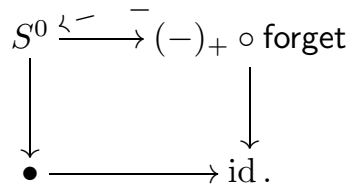

Furthermore we remark that this is a cofibre diagram in Space. Applying the functor $\Sigma^{\infty}$ (which has a right adjoint and hence preserves small colimits by Lurb, Proposition 5.2.3.5] ) we obtain a natural bi-cartesian diagram of Sp-valued functors Space. $\rightarrow$ Sp, with a canonical splitting

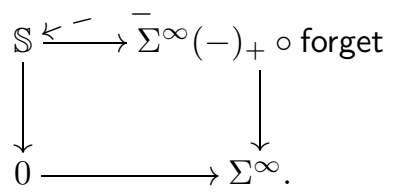

We conclude that there is a natural equivalence $\Sigma^{\infty}(-)_{+} \circ$ forget $\simeq \mathbb{S} \oplus \Sigma^{\infty}$ of Sp-valued functors. 
Specialising this to the pointed space $\left(S^{1}, 1\right)$ we obtain the equivalence:

Corollary 4.13. $\Sigma_{+}^{\infty} S^{1} \simeq \mathbb{S} \oplus \Sigma \mathbb{S}$.

The following assertion lies at the heart of the definition of higher commutators for spectral extensions.

Corollary 4.14. The equivalence of Corollary 4.12 induces for a pointed space $\mathcal{X}=\left(X, x_{0}\right)$ a natural morphism and a natural left inverse thereof

$$
\Sigma^{\infty} \mathcal{X}^{\wedge n} \leftrightarrows \Sigma_{+}^{\infty}\left(X^{n}\right)
$$

Proof. We have recorded in (e) above that $\Sigma_{+}^{\infty}$ is symmetric monoidal. Hence for every positive integer $n$, and every unpointed space $X$ we get a contractible space of morphisms

$$
\Sigma_{+}^{\infty}\left(X^{n}\right) \rightarrow\left(\Sigma_{+}^{\infty} X\right)^{\otimes n} .
$$

For $\mathcal{X}=\left(X, x_{0}\right)$ we can draw on the natural equivalence of functors $\Sigma^{\infty}(-)_{+} \simeq$ $\mathbb{S} \oplus \Sigma^{\infty}(-)$ for $\mathcal{X}$, which allows us to define a natural morphism with left inverse

$$
\left(\Sigma_{+}^{\infty} X\right)^{\otimes n} \leftrightarrows \Sigma^{\infty} \mathcal{X}^{\wedge n}
$$

Here we used repeatedly that $\otimes$ commutes with small colimits in its entries.

We observe the following:

Remark 4.15 . The corollary above can be refined to produce a natural equivalence

$$
\Sigma_{+}^{\infty}\left(X^{n}\right) \simeq \oplus_{i=0}^{n}\left(\Sigma^{\infty} \mathcal{X}^{\wedge i}\right)^{\oplus\left(\begin{array}{c}
n \\
i
\end{array}\right)}
$$

for every pointed space $\mathcal{X}=\left(X, x_{0}\right)$.

The last conclusion we draw is again rather general.

Corollary 4.16. The functor $\Sigma_{+}^{\infty}:$ Space $\rightarrow$ Sp sends $E_{\infty}$-objects in (unpointed) spaces to $E_{\infty}$-ring spectra.

Proof. It is a general statement that symmetric monoidal functors preserve $E_{\infty}$ objects (also called commutative algebra objects in [Lura]). This follows directly from the definitions, we give the proof since we could not find a reference. In the notation of [Lura, Chapter 2], a symmetric monoidal structure on an $\infty$-category is encoded by a functor $\mathrm{C}_{\otimes} \rightarrow N\left(\mathrm{Fin}_{*}\right)$ satisfying certain properties (see Lura, Definition 2.0.0.7]). A symmetric monoidal functor is given by a commutative diagram (see Lura] for a precise account of further technical conditions required from the functor)

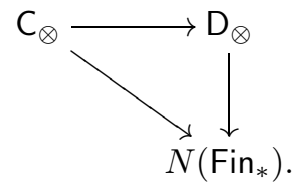

On the other hand, a commutative algebra object in $\mathrm{C}_{\otimes}$ is encoded by a section $\mathrm{C}_{\otimes} \leftrightarrows N\left(\mathrm{Fin}_{*}\right)$ which is a map of $\infty$-operads (see Lura, Definition 2.1.3.1]). It is therefore clear that a symmetric monoidal functor sends such a section for $\mathrm{C}_{\otimes} \rightarrow$ $N\left(\operatorname{Fin}_{*}\right)$ to one for $\mathrm{D}_{\otimes}$. 
4.3.4. The definition of higher commutators. Let $X$ be a groupoid, that is a category where all morphisms are invertible. The groupoid $X$ gives rise to an unpointed space, namely the geometric realization of its nerve $|N X|$. Henceforth this will be implicit, and we use this construction to realize the 2-category of groupoids as a full subcategory of the $\infty$-category of unpointed spaces Space.

Every group $G$ gives rise to a groupoid $B G$, by definition the category with a unique object $\{*\}$ and $\operatorname{Aut}_{B G}(*)=G$. Consistent with the paragraph above, we also denote the associated unpointed space by $B G$. However we remark that the functor $B:$ Grps $\rightarrow$ Space factors through the $\infty$-category of pointed spaces Space:

$$
\mathcal{B}: G \mapsto(B G, *) .
$$

Let $\mathbb{E}$ be a spectrum, and $X$ a groupoid. A spectral extension of $X$ by $\mathbb{E}$ is defined to be a morphism $\Sigma_{+}^{\infty} X \rightarrow \Sigma^{2} \mathbb{E}$. In particular, for $G$ a group, a spectral extension of $G$ by $\mathbb{E}$ is given by a morphism $e: \Sigma_{+}^{\infty} B G \rightarrow \Sigma^{2} \mathbb{E}$.

Definition 4.17. Let $x \in X$ be an object. We denote by $P_{X}^{n}$ the groupoid whose objects are tuples $\left(x ; g_{1}, \ldots, g_{n}\right)$ where $x \in X$ is an object, and $\left(g_{1}, \ldots, g_{n}\right) \in$ $\left(\operatorname{Aut}_{X}(x)\right)^{n}$ is an $n$-tuple of pairwise commuting automorphisms. Morphisms $\left(x ; g_{1}, \ldots, g_{n}\right) \rightarrow\left(y ; h_{1}, \ldots, h_{n}\right)$ in $P_{X}^{n}$ are given by a morphism $f: x \rightarrow y$, such that $f \circ g_{i}=h_{i} \circ f$ for all $1 \leq i \leq n$. This defines an endofunctor $P_{n}$ of the 2-category of groupoids Grpd.

Objects of $P_{X}^{n}$ are pairwise commuting $n$-tuples of automorphisms in $X$ at a fixed object $x$. It follows that there is a natural equivalence $P_{X}^{n} \simeq \operatorname{Map}\left(\left(B \mathbb{Z}^{n}\right)_{+}, X_{+}\right)$.

Lemma 4.18. With respect to the embedding of groupoids in unpointed spaces, the functor

$$
P^{n}: \text { Grpd } \rightarrow \text { Grpd }
$$

is naturally equivalent to $\operatorname{Map}_{\text {space }}\left(B \mathbb{Z}^{n}, X\right)$.

Proof. We obtain the object $x$ as the image of $*$ under the corresponding map of unpointed spaces $B \mathbb{Z}^{n} \rightarrow X$. Since $\operatorname{Aut}_{B \mathbb{Z}^{n}}\left(x_{0}\right)=\mathbb{Z}^{n}$ we have a canonical choice for an $n$-tuple of pairwise commuting automorphisms, given by the standard basis of $\mathbb{Z}^{n}$. We then transport this choice along the induced map of groups $\mathbb{Z}^{n} \rightarrow \operatorname{Aut}_{X}(x)$ to conclude the proof.

Recall that $B \mathbb{Z}^{n}$ is a strict abelian group object in Space. We obtain from Corollary 4.16 that $\Sigma_{+}^{\infty}\left(B \mathbb{Z}^{n}\right)$ is an $E_{\infty}$-ring spectrum. In particular we see that $\pi_{*}\left(\Sigma_{+}^{\infty}\left(B \mathbb{Z}^{n}\right)\right)$ is a graded commutative algebra. We will write $(x \mid y)$ to denote the product of two elements, and more generally $\left(x_{1}|\cdots| x_{n}\right)$ for the product of $n$ elements.

Definition 4.19. Let $e: \Sigma_{+}^{\infty} X \rightarrow \Sigma^{2} \mathbb{E}$ be a spectral extension of a groupoid $X$ by a spectrum $\mathbb{E}$.

(a) Corollary 4.12 applied to the pointed space $\left(S^{1}, 1\right)=(B \mathbb{Z}$, *) yields a splitting $\Sigma_{+}^{\infty} B \mathbb{Z} \simeq \mathbb{S} \oplus \Sigma \mathbb{S}$. The map of spectra $\Sigma \mathbb{S} \rightarrow \Sigma_{+}^{\infty} B \mathbb{Z}$ will be denoted by $f$.

(b) For an integer $i$ satisfying $1 \leq i \leq n$ we let $\mathbb{Z} \rightarrow \mathbb{Z}^{n}$ be the map $\lambda \mapsto$ $(0, \ldots, 0, \lambda, 0, \ldots, 0)$ given by the inclusion of the $i$-th component. We write $\phi_{i}: B \mathbb{Z} \rightarrow B \mathbb{Z}^{n}$ for the induced map of pointed spaces. The induced element $\left(\phi_{i}\right)_{*}(f) \in \pi_{1}\left(\Sigma_{+}^{\infty} B \mathbb{Z}^{n}\right)$ is denoted by $e_{i}$. 
(c) Let $\left(B \mathbb{Z}^{n}\right) \stackrel{g}{\rightarrow} X$ be an $n$-tuple of pairwise commuting automorphisms. We denote the induced map of spectra $\Sigma_{+}^{\infty}\left(B \mathbb{Z}^{n}\right) \stackrel{g}{\rightarrow} \Sigma_{+}^{\infty}(X) \stackrel{e}{\rightarrow} \Sigma^{2} \mathbb{E}$ by $\phi(g, e)$. The higher commutator is defined to be $\phi(g, e)_{*}\left(e_{1}|\cdots| e_{n}\right) \in \pi_{n-2}(\mathbb{E})$ and will be denoted by $g_{1} *_{e} \cdots *_{e} g_{n}$.

4.3.5. Comparison. In order to show that this definition is non-trivial we compare it to the construction of Definition 4.8 .

Lemma 4.20. Let $G$ be a group, and let $[e] \in H_{g r p}^{n+2}(G, A)$ be a higher central extension corresponding to a map e: $B G \rightarrow B^{n+2} A$ and let $\left(g_{0}, \ldots, g_{n+1}\right)$ be an $(n+$ $2)$-tuple of pairwise commuting elements. With respect to the natural isomorphism $\pi_{n}(K(A, n)) \simeq A$ we have that the spectral higher commutator $g_{0} *_{e} \cdots *_{e} g_{n+1}$ agrees with the higher commutator $\left(g_{0}, \ldots, g_{n+1}\right)_{e}$ of Definition 4.8 .

Proof. Recall that for an unpointed space $X$ we have the Hurewicz morphism $h: \pi_{*}\left(\Sigma_{+}^{\infty} X\right) \rightarrow H_{*}(X, \mathbb{Z})$, obtained by applying the functor $\pi_{*}$ to the morphism of spectra

$$
\Sigma_{+}^{\infty} X \rightarrow \Sigma_{+}^{\infty} X \otimes \mathbb{Z}
$$

where we use the notation $\mathbb{Z}$ to denote the Eilenberg-Mac Lane spectrum corresponding to the ring $\mathbb{Z}$.

If $X$ is an $E_{\infty}$-object in (unpointed) spaces (that is, a commutative monoid), we have already seen that $\pi_{*}\left(\Sigma_{+}^{\infty} X\right)$ and $H_{*}(X, \mathbb{Z})$ are endowed with a graded commutative product structure. The Hurewicz morphism respects this product, since the morphism (42) is a morphism of $E_{\infty}$-ring spectra, induced by the morphism of $E_{\infty}$-ring spectra $\mathbb{S} \rightarrow \mathbb{Z}$.

We have a commutative diagram of abelian groups

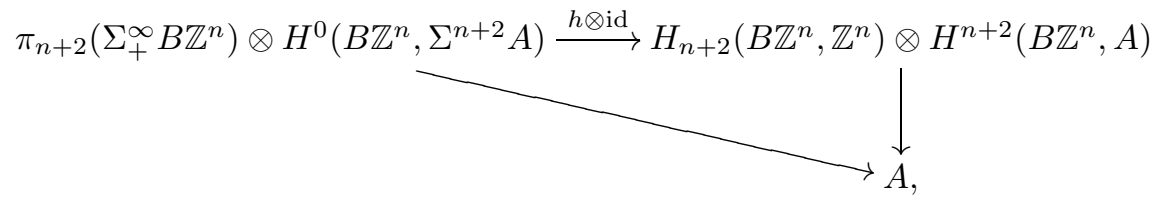

and furthermore we have $h\left(e_{0}|\cdots| e_{n+1}\right)=\left(e_{0}|\ldots| e_{n+1}\right)$ by the discussion above. By virtue of Definitions 4.19 and 4.8 we conclude that the new notions of higher commutators agree.

4.3.6. Computing higher commutators recursively. For a group $G$, and $g \in G$, we write $C_{G}(g)$ to denote the centralizer. We now describe a version of the classical slash product to associate to a spectral extension $e: \Sigma_{+}^{\infty} X \rightarrow \Sigma^{2} \mathbb{E}$ and an element $g \in \operatorname{Aut}_{X}(x)$, a spectral extension $e\langle g\rangle: \Sigma_{+}^{\infty} B C_{\mathrm{Aut}_{X}(x)}(g) \rightarrow \Sigma^{2}(\Omega \mathbb{E})$.

Definition 4.21. Let $X$ be a groupoid, $x \in X$ an object, and $g \in \operatorname{Aut}_{X}(x)=G$ an automorphism. We denote by $e: \Sigma_{+}^{\infty} X \rightarrow \Sigma^{2} \mathbb{E}$ a spectral extension of $X$ by $\mathbb{E}$.

(a) We let $\left(B C_{\operatorname{Aut}_{X}(x)}(g)\right) \rightarrow X$ be the map of unpointed spaces induced by the inclusion $C_{\mathrm{Aut}_{X}(x)}(g) \subset \operatorname{Aut}_{X}(x)$.

(b) Let $B \mathbb{Z} \times B C_{\mathrm{Aut}_{X}(x)}(g) \rightarrow B G$ be the map of unpointed spaces induced by the map of groups $\mathbb{Z} \times C_{\text {Aut }_{X}(x)}(g) \rightarrow G$ sending $1 \in \mathbb{Z}$ to $g$, and given by the inclusion of $C_{\mathrm{Aut}_{X}(x)}(g)$.

(c) We denote by $\Sigma \mathbb{S} \rightarrow \Sigma_{+}^{\infty} B \mathbb{Z} \simeq \Sigma_{+}^{\infty} S^{1}$ the map specified by Corollary 4.13 . 
(d) The map $e\langle g\rangle: \Sigma_{+}^{\infty} B C_{\mathrm{Aut}_{X}(x)}(g) \rightarrow \Sigma^{2}(\Omega \mathbb{E})$ is defined to be the adjoint to the map

$$
\Sigma \Sigma_{+}^{\infty} B C_{\text {Aut }_{X}(x)}(g) \rightarrow \Sigma^{2} \mathbb{E}
$$

defined by the composition

$$
\begin{aligned}
& \Sigma \Sigma_{+}^{\infty} B C_{\mathrm{Aut}_{X}(x)}(g) \\
& \quad \simeq \Sigma \mathbb{S} \otimes \Sigma_{+}^{\infty} B C_{\mathrm{Aut}_{X}(x)}(g) \rightarrow \Sigma_{+}^{\infty}\left(B \mathbb{Z} \times B C_{\mathrm{Aut}_{X}(x)}(g)\right) \rightarrow \Sigma_{+}^{\infty} G \rightarrow \Sigma^{2} \mathbb{E},
\end{aligned}
$$

where we have used that $\Sigma_{+}^{\infty}$ is symmetric monoidal as explained in (i) above.

The following assertion follows right from the definitions.

Lemma 4.22. Let $\left(g_{1}, \ldots, g_{n}\right) \in P_{n}(G)$. Then we have $\left(g_{1} \star \cdots \star g_{n}\right)_{e} \cong\left(g_{2} \star \cdots \star\right.$ $\left.g_{n}\right)_{e\left\langle g_{1}\right\rangle} \cdot$

4.3.7. Comparison with Osipov-Zhu's definition for $n=3$. Recall that a groupoid endowed with a symmetric monoidal structure is called a Picard groupoid, if the monoidal structure is group-like. That is, the induced monoid structure on the set of isomorphism classes is a group structure. We denote by $\mathbf{P}$ a Picard groupoid, and by $\mathbf{0} \in \mathbf{P}$ a unit. The group $\operatorname{Aut}_{\mathbf{P}}(0)$ is abelian (as a consequence of the EckmannHilton trick), and will be denoted by $\boldsymbol{\Omega P}$. Similar conventions will be applied to Picard 2-groupoids, that is, group-like symmetric monoidal $(2,1)$-groupoids.

Given a spectral extension $e$ of $G$ by $\mathbb{E}$ and an element $g \in G$, we constructed (see Definition 4.21) a spectral extension $e\langle g\rangle$ of the centralizer $C(g)$ by $\Omega \mathbb{E}$. This shifted spectral extension satisfies the identity (Lemma 4.22)

$$
\left(g_{1} \star \cdots \star g_{n}\right)_{e} \cong\left(g_{2} \star \cdots \star g_{n}\right)_{e\left\langle g_{1}\right\rangle} .
$$

Readers of Osipov-Zhu's OZ11 will recognize the similarity with their recursive definition of higher commutators. The authors of loc. cit. associate to an extension $\psi$ of a group $G$ by a Picard groupoid $\mathbf{P}$, and an element $f \in G$ a (graded) central extension $\psi_{f}$ of $C(f)$ by $\operatorname{Aut}_{\mathbf{P}}(\mathbf{0})$. Eventually, the commutator $C_{3}(f, g, h)$ is defined to be $\operatorname{Comm}\left(\psi_{f}\right)(g, h)$ with respect to the latter central extension. Here $\operatorname{Comm}\left(\psi_{f}\right)$ denotes the commutator pairing of [OZ11, Lemma-Definition 2.5].

Proposition 4.23. Let $\phi: G \rightarrow B \mathbf{P}$ be the monoidal map corresponding to a central extension of $G$ by $\mathbf{P}$. We denote by $e: \Sigma^{\infty} B G \rightarrow \Sigma \mathbb{B} \mathbf{P}$ the corresponding spectral extension of $G$ by $\Omega \mathbb{B} \mathbf{P}$, the spectrum associated to the Picard groupoid $\mathbf{P}$. Then,

$$
C_{3}(f, g, h)=f \star g \star h .
$$

Proof. At first we recall Osipov-Zhu's construction of the central extension of the centralizer $C(f)$ by $\Omega^{2} \mathbf{P}$. In OZ11, Lemma-Definition 2.13] they define a symmetric monoidal map

$$
C(f) \rightarrow \mathbf{P},
$$

which sends $g \in C(f)$ to the element of $\mathbf{P} \simeq A u t_{B \mathbf{P}}(\mathbf{0})$ given by

$$
\phi(f g) \cong \phi(f) \phi(g) \cong \phi(g) \phi(f) \cong \phi(g f) \cong \phi(f g) .
$$

It is well-known that the $(3,1)$-category of group-like symmetric monoidal $(2,1)$ groupoids is equivalent to the full subcategory $\mathrm{Sp}_{[0,2]}$ of the $\infty$-category of spectra $\mathrm{Sp}$, consisting of spectra $\mathbb{E}$ with vanishing $\pi_{i}(\mathbb{E})$ for $i \notin[0,2]$. This assertion can be deduced from a result of Boardman-Vogt and May (see [Lura, Theorem 5.2.6.10]). 
This equivalence allows one to consider $\mathbf{P}$ as a spectrum, which we denote $\Omega \mathbb{B} \mathbf{P}$. Osipov-Zhu's map (43) is then an explicit description of the adjoint to

$$
\Sigma \Sigma_{+}^{\infty} B C(f) \simeq \Sigma_{+}^{\infty}(B \mathbb{Z} \times B C(f)) \rightarrow \mathbb{B} \mathbf{P},
$$

and hence is equivalent to the central extension $e\langle f\rangle$ defined in Definition 4.21. We infer the following assertion:

Claim 4.24. The symmetric monoidal map $\psi_{f}: C(f) \rightarrow \mathbf{P}$ defined in OZ11, LemmaDefinition 2.13] is homotopic to the map $e\langle f\rangle$ of Definition 4.21, with respect to the natural embedding of Picard groupoids into the $\infty$-category of spectra.

It remains to compare Osipov-Zhu's $\operatorname{Comm}(\psi)(g, h)$ of OZ11, Lemma-Definition $2.5]$ with $g *_{e\langle f\rangle} h$. This is the content of the next assertion.

Claim 4.25. Let $H$ be a group, $\mathbf{P}$ a Picard groupoid, and $\psi: H \rightarrow \mathbf{P}$ a monoidal morphism. We denote by $\alpha$ the corresponding spectral extension of $H$ by $\Omega \mathbb{B} \mathbf{P}$. Then we have for $(g, h) \in P_{2}(H)$ the equality $\operatorname{Comm}(\psi)(g, h)=g *_{\alpha} h$ of elements of $\operatorname{Aut}_{\mathbf{P}}(\mathbf{0})$.

For any $g \in C(f)$, we have that the map $\left(\psi_{f}\right)_{g}: C(g) \rightarrow \operatorname{Aut}_{\mathbf{P}}(\mathbf{0})$ of OZ11, Lemma-Definition 2.5] is homotopic to $\alpha\langle g\rangle$ (by an argument analogous to the one above, one category level down).

To deduce Claim 4.25, observe that it follows directly from the definition given in OZ11, Lemma-Definition 2.5] that $\operatorname{Comm}(\psi)(g, h)=\psi_{g}(h)$, and similarly, we know by virtue of Lemma 4.22 that $\alpha\langle g\rangle(h)=g *_{\alpha} h$. We deduce

$$
\operatorname{Comm}(\psi)(g, h)=g *_{\alpha} h .
$$

This concludes the proof of Claim 4.25. The proposition follows.

4.4. Spectral extensions coming from the K-theory of rings. We begin with a quick review of the relevant facts about $K$-theory. This will also serve to fix notation. Experts should feel free to skip ahead.

4.4.1. Steinberg symbols. In the following we denote by $R$ a ring, again assumed commutative and unital. Careful inspection of the definition of your choice of algebraic $K$-theory, reveals the existence of a canonical morphism

$$
\Sigma_{+}^{\infty} \coprod_{n \in \mathbb{N}_{\geq 1}} B \mathrm{GL}_{n}(R) \rightarrow \mathbb{K}_{R}
$$

More generally, for a stable $\infty$-category $\mathrm{C}$, there is a canonical morphism

$$
\Sigma_{+}^{\infty} \mathrm{C}^{\times} \rightarrow \mathbb{K}_{\mathrm{C}}
$$

The morphism (44) is a special case of this construction 25

Definition 4.26. The existence of the morphism (44) can be restated as saying that the groupoid $\coprod_{n \in \mathbb{N}} B \mathrm{GL}_{n}(R)$ is canonically endowed with a central extension by $\Omega^{2} \mathbb{K}_{R}$. Similarly, (45) amounts to the $\infty$-groupoid $C^{\times}$being endowed with a central extension by $\Omega^{2} \mathbb{K}_{\mathrm{C}}$. We will denote the extensions by $e_{R}$ and $e_{\mathrm{C}}$ respectively.

The central extension of $\mathrm{GL}_{n}(R)$ by $\Omega^{2} \mathbb{K}_{R}$ has appeared in work of Safronov Saf16. The theory of higher commutators developed in this section enables us to generalize Steinberg symbols to a general stable $\infty$-category.

\footnotetext{
${ }^{25}$ I.e. after factoring through the inclusion $P_{f}(R)^{\times} \rightarrow \operatorname{Perf}(R)^{\times}$.
} 
Definition 4.27. We denote by $\left(g_{1}, \ldots, g_{n}\right) \in P_{n}\left(\mathrm{C}^{\times}, x\right)$ a map of unpointed spaces $\mathbb{T}^{n} \rightarrow \mathrm{C}^{\times}$mapping the base point of $\mathbb{T}^{n}$ to $x$. The map

$$
\left(g_{1} \star \cdots \star g_{n}\right)_{e_{\mathrm{C}}}: \Sigma^{\infty} \mathbb{S}_{+}^{n} \rightarrow \mathbb{K}_{\mathrm{C}}
$$

is referred to as the higher commutator with respect to the natural extension of $\mathrm{C}^{\times}$ by $\Omega^{2} \mathbb{K}_{C}$.

The justification of the terminology Steinberg symbol is provided by the next proposition, which compares the higher commutators of Definition 4.27 with Loday's higher Steinberg symbols, for the category of finitely generated projective $R$-modules.

Proposition 4.28. Let $R$ be a commutative ring, and $r_{1}, \ldots, r_{n} \in R^{\times}$be an $n$ tuple of units in $R$. The higher commutator $\left(r_{1} \star \cdots \star r_{n}\right)_{e_{R}}$, computed with respect to the spectral extension $e_{R}$ of Definition 4.26, agrees with Loday's higher Steinberg symbol $\left\{r_{1}, \ldots, r_{n}\right\}$.

Before giving the proof, we recall Loday's definition from Lod76. In modern language, Loday's construction of the Steinberg symbols relies on the $E_{\infty}$-ring structure of $K_{R}$ (in which the product is induced by the tensor product $\otimes$ of $R$-modules). If $\alpha_{1}, \ldots, \alpha_{n}$ is an $n$-tuple of paths in $K_{R}$ based at $\mathbf{0} \in K_{R}$, the multiplication $\otimes$ induces a map

$$
\Sigma^{\infty}\left(\mathbb{S}^{1}\right)^{\wedge n} \rightarrow \mathbb{K}_{R}^{\wedge n} \rightarrow \mathbb{K}_{R}
$$

which defines an element $\left(\alpha_{1}|\ldots| \alpha_{n}\right)$ of $\pi_{n}\left(\mathbb{K}_{R}\right)=K_{n}(R)$.

Proof of Proposition 4.28, Let $\mathrm{C}$ be an exact category with a bi-exact symmetric monoidal structure $\otimes$. This endows the maximal pointed groupoid $\mathrm{C}^{\times}$with a symmetric monoidal structure $\otimes$. By definition, the canonical map $\Sigma_{+}^{\infty} C^{\times} \rightarrow \mathbb{K}_{C}$ is a map of $E_{\infty}$-ring spectra.

For $\mathrm{C}=P_{f}(R)$ the symmetric monoidal exact category of finitely generated projective $R$-modules, we have a symmetric monoidal morphism $B R^{\times} \rightarrow\left(P_{f}(R)\right)^{\times}$. It is obtained by viewing $\left(B R^{\times}, \otimes\right)$ as the symmetric monoidal category of free $R$ modules of rank 1 . Therefore we have a morphism $B R^{\times} \rightarrow P_{f}(R)^{\times}$of $E_{\infty}$-objects in (unpointed) spaces. By virtue of Corollary 4.16 we obtain a morphism of $E_{\infty}$-ring spectra

$$
\Sigma_{+}^{\infty} B R^{\times} \rightarrow \Sigma_{+}^{\infty} P_{f}(R)^{\times} .
$$

For $\left(r_{1}, \ldots, r_{n}\right) \in R^{\times}$the resulting map $B \mathbb{Z}^{n} \rightarrow B R^{\times}$is symmetric monoidal, and therefore, another application of Corollary 4.16 yields a morphism of $E_{\infty}$-ring spectra

$$
\Sigma_{+}^{\infty} B \mathbb{Z}^{n} \rightarrow \Sigma_{+}^{\infty} B R^{\times}
$$

Composing the morphism of $E_{\infty}$-ring spectra defined above, we obtain

$$
\psi: \Sigma_{+}^{\infty} B \mathbb{Z}^{n} \rightarrow \Sigma_{+}^{\infty} P_{f}(R)^{\times} \rightarrow \mathbb{K}_{R} .
$$

Recall that we have a functor from the homotopy category of $E_{\infty}$-ring spectra to the category of graded commutative rings. This implies the equality $\psi_{*}\left(e_{1}|\cdots| e_{n}\right)=$ $\left(r_{1}|\ldots| r_{n}\right)$, where we denote by $\left(e_{1}, \ldots, e_{n}\right) \in \mathbb{Z}^{n}$ the standard basis of $\mathbb{Z}^{n}$. By definition of higher commutators, the left hand side agrees with $r_{1} * \cdots * r_{n}$. The right hand side on the other hand is given by Loday's higher Steinberg symbol $\left\{r_{1}, \ldots, r_{n}\right\}$. This concludes the proof. 


\section{The CC symbol via Tate categories}

Now we are almost ready give the full definition of our higher Contou-Carrère symbol, pursuing the strategy which had called Idea 2 in the introduction.

\subsection{Lattices and Tate objects.}

5.1.1. Tate objects in exact categories. We recall the constructions of Ind, Pro, and Tate objects in exact categories, and refer the reader to BGW16c for background. The ideas of these constructions go back to papers by Beilinson Bei87 and Kato Kat00, and have also been studied by Previdi in [Pre11. We also refer the reader to Drinfeld's theory of Tate $R$-modules introduced in Dri06.

A filtered set $I$ is a set $I$ together with a partial ordering $\leq$, such that for each pair $(i, j) \in I^{2}$ there exists a $k \in I$, satisfying $i \leq k$ and $j \leq k$. Every filtered set can be viewed as a category in a straightforward manner.

Let $C$ be an exact category. An admissible Ind-object in $C$ indexed by $I$ is a functor $X: I \rightarrow \mathrm{C}$, such that the relation $i \leq j$ determines an admissible monomorphism with respect to the exact structure of $\mathrm{C}$. For example, we can take $I$ to be the set $\mathbb{N}$ with its natural ordering. An $\mathbb{N}$-indexed admissible Ind-object in $C$ can then be pictured as a formal colimit of a diagram

$$
X_{0} \hookrightarrow X_{1} \hookrightarrow X_{2} \hookrightarrow \cdots .
$$

Every admissible Ind-object gives rise to a left exact presheaf. To $X: I \rightarrow \mathrm{C}$ one associates the presheaf $A \mapsto \lim _{i \in I} \operatorname{Hom}(A, X(i))$. The resulting full subcategory of Lex $(C)$ of all objects of this shape is denoted by $\operatorname{Ind}^{a}(\mathrm{C})$. In Theorem 3.7 of BGW16c the authors showed that $\operatorname{Ind}^{a}(\mathrm{C})$ is an extension closed subcategory of Lex(C). This implies that it inherits a structure of an exact category.

Admissible Pro-objects in $\mathrm{C}$ are defined dually, i.e. by replacing the role of admissible monomorphisms by admissible epimorphisms. In short we have, $\operatorname{Pro}^{a}(\mathrm{C})=$ $\left(\text { Ind }^{a}\left(\mathrm{C}^{\mathrm{op}}\right)\right)^{\mathrm{op}}$. An admissible Pro-object indexed by a filtered set $I$ is a functor $X: I^{\text {op }} \rightarrow \mathrm{C}$, which sends $i \leq j$ to an admissible epimorphism in C. For $I=\mathbb{N}$ we obtain the dual depiction of a Pro-object as a formal limit of a diagram

$$
X_{0} \leftarrow X_{1} \leftarrow X_{2} \leftarrow \cdots .
$$

An elementary Tate object is an admissible Ind-Pro-object, i.e. an object $V$ in Ind $^{a} \operatorname{Pro}^{a}(\mathrm{C})$, which can be (non-canonically) written as an extension

$$
L \hookrightarrow V \rightarrow V / L,
$$

with $L \in \operatorname{Pro}^{a}(\mathrm{C})$ and $V / L \in \operatorname{Ind}^{a}(\mathrm{C})$. We refer to any such $L$ as a lattice in $V$. The category of elementary Tate objects in $\mathrm{C}$ has a natural exact structure (Theorem 5.4 in [BGW16c] , and will be denoted by $\operatorname{Tate}^{e l}(\mathrm{C})$.

Proposition 5.1 (Kapranov). If $k$ is a field, then $\operatorname{Tate}^{e l}\left(\mathrm{Vect}_{k}^{f d}\right)$, i.e. the exact category of elementary Tate objects of finite-dimensional $k$-vector spaces, is equivalent to the category of locally linearly compact topological $k$-vector spaces (as exact categories).

See Kap01, §1.1.2].

The exact category Tate $(C)$ of Tate objects in $C$ is defined to be the idempotent completion of Tate ${ }^{e l}(\mathrm{C})$. If $R$ is a ring, and $\mathrm{C}=P_{f}(R)$, the exact category of finitelygenerated projective $R$-modules, then Tate $\left(P_{f}(R)\right)$ contains Drinfeld's category of 
Tate $R$-modules as a full subcategory. See [BGW16c, Thm. 5.26], where we show that for countable index sets $I$, the two categories are in fact equivalent. We emphasize that in [Dri06], Drinfeld refers to what we call lattices as co-projective lattices.

Definition 5.2. For a category D (respectively $\infty$-category), we denote by $D^{\times}$the maximal groupoid contained in D (respectively $\infty$-groupoid).

The following result is BGW18b, Prop. 3.3].

Proposition 5.3. For an idempotent complete exact category $\mathrm{C}$, we denote by $G r \leq(\mathrm{C})$ the simplicial object in groupoids, which parametrizes chains $\left(V \supset L_{n} \supset\right.$ $\left.\cdots \supset L_{0}\right)$, where $V$ is an elementary Tate object in $\mathrm{C}$, and each $L_{i}$ is a lattice in $V$. We have a forgetful morphism $\mathrm{Gr}_{\bullet}(\mathrm{C}) \rightarrow \mathrm{Tate}^{\text {el }}(\mathrm{C})^{\times}$, which induces an equivalence

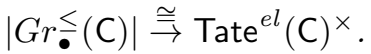

5.1.2. The index map. Let $\mathrm{C}$ be an exact category, following Waldhausen Wal85] we denote by $S_{n}(\mathrm{C})$ the exact category, whose objects correspond to chains

$$
X_{1} \hookrightarrow \cdots \hookrightarrow X_{n}
$$

of admissible monomorphisms (plus a fixed choice for all possible quotients among these objects). The $S_{n}(\mathrm{C})$ fit together to give a simplicial object $S_{\bullet}$ (C) in the 2 category of exact categories: face maps are given by omitting an object/composing maps, and degeneracies by inserting the identity map.

Waldhausen's treatment of algebraic $K$-theory in Wal85 implies that, for an exact category $\mathrm{C}$, the classifying space $B K_{\mathrm{C}}$ is equivalent to the geometric realization of the simplicial object in groupoids $\left|S_{\bullet} \mathrm{C}^{\times}\right|$.

Now let $\mathrm{C}$ be an idempotent complete exact category, and let $G r_{\bullet}(\mathrm{C})$ be as in Proposition 5.3 .

Definition 5.4. Let Index: $G r_{\bullet}^{\leq}(\mathrm{C}) \rightarrow S_{\bullet}(\mathrm{C})$ be the map sending $\left(V \supset L_{n} \supset \cdots \supset\right.$ $\left.L_{0}\right)$ to $\left(L_{1} / L_{0} \hookrightarrow \cdots \hookrightarrow L_{n} / L_{0}\right)$. Whenever convenient,

- the geometric realization Tate ${ }^{e l}(\mathrm{C})^{\times} \rightarrow B K_{\mathrm{C}}$, as well as

- the induced map $K_{\text {Tate }^{e l}(\mathrm{C})} \rightarrow B K_{\mathrm{C}}$ (see [BGW18b, Cor. 3.5])

will also be denoted Index and called the index map as well.

This is the map which we had alluded to in the introduction of the paper, see Equation (27).

For every elementary Tate object $V$, we obtain from

$$
B \operatorname{Aut}(V) \stackrel{\alpha}{\rightarrow} \operatorname{Tate}^{e l}(\mathrm{C}) \stackrel{\times \text { Index }}{\rightarrow} B K_{\mathrm{C}},
$$

a monoidal map

$$
\operatorname{Aut}(V) \rightarrow K_{\mathrm{C}}
$$

by applying the loop space functor $\Omega$. Above, the map $\alpha$ is the one coming from the construction of Remark 2.8 . 


\subsection{The classical Contou-Carrère symbol.}

5.2.1. The Contou-Carrère symbol. We had recalled the classical tame symbol in Equation (13). The Contou-Carrère symbol arises as a "deformation" of the tame symbol for the discrete valuation ring $R=k((t))$. For every (commutative) $k$ algebra $A$, we can consider the ring of formal Laurent series $A((t))$, which is almost never a discrete valuation ring. Nonetheless, there exists a natural pairing $A((t))^{\times} \times$ $A((t))^{\times} \rightarrow A^{\times}$, which specializes to the tame symbol for the case $A=k$. For $A \neq k$, the explicit formula (13) no longer holds. However the interpretation of the tame symbol as a graded commutator [ADCK89] remains valid for Contou-Carrère symbols by work of Anderson-Pablos Romo APR04 and Beilinson-Bloch-Esnault BBE02. We hence begin by summarizing the definition using graded commutators.

We denote by $\mathcal{V}_{A}$ the Tate object $A((t))$ in Tate $\left(P_{f}(A)\right)$, see BGW16b, Example 10] for a precise definition. There is a natural map $A((t))^{\times} \rightarrow \operatorname{Aut}\left(\mathcal{V}_{A}\right)$. Let $\mathbb{B}^{\mathbb{Z}} \mathbb{G}_{m}(A)$ denote the spectrum associated to the Picard groupoid of graded $A$-lines. For each $A$, the index map and determinant give rise to a spectral extension

$$
\Sigma_{+}^{\infty} B\left(A((t))^{\times}\right) \rightarrow \Sigma_{+}^{\infty} B \operatorname{Aut}\left(\mathcal{V}_{A}\right) \rightarrow \mathcal{K}_{\text {Tate }^{e l}\left(P_{f}(A)\right)} \stackrel{\text { Index }}{\rightarrow} \Sigma \mathcal{K}_{P_{f}(A)} \stackrel{\text { det }}{\rightarrow} \Sigma \mathbb{B}^{\mathbb{Z}} \mathbb{G}_{m}(A) .
$$

Looping the adjoint of this map yields an $E_{1}$-map

$$
A((t))^{\times} \rightarrow B^{\mathbb{Z}} \mathbb{G}_{m}(A),
$$

classifying a graded central extension of $A((t))^{\times}$. The construction is natural in maps $A \rightarrow A^{\prime}$, so it defines a central extension of group-valued sheaves. We record this observation in the following definition. Recall that the loop group $L \mathbb{G}_{m}$ is defined as the group-valued presheaf

$$
L \mathbb{G}_{m}:\left(\mathrm{Aff}_{k}\right)^{\mathrm{op}} \rightarrow \mathrm{Grp}
$$

sending $A$ to $A((t))^{\times}$.

Definition 5.5. The graded central extension (50) of $L \mathbb{G}_{m}$ will be denoted by

$$
\phi_{K M}: L \mathbb{G}_{m} \rightarrow B^{\mathbb{Z}} \mathbb{G}_{m},
$$

and referred to as the Kac-Moody extension of the loop group. We denote the spectral Kac-Moody extension (49) by

$$
e_{s K M}: \Sigma_{+}^{\infty} B L \mathbb{G}_{m} \rightarrow \Sigma \mathcal{K},
$$

where $\mathcal{K}$ denotes the presheaf in connective spectra, sending a $\operatorname{ring} A$ to $\mathcal{K}_{A}$.

Note that the Kac-Moody extension is obtained from the spectral Kac-Moody extension by looping and applying the determinant.

We can now recall the following well-known result, which generalizes the main result of the paper APR04 to arbitrary $k$-algebras (without restricting to the artinian case).

Proposition 5.6. The graded central extension $\phi_{K M}$ of Definition 5.5 relates to the Contou-Carrère symbol by means of the relation

$$
(-,-)^{-1}=-\star_{\phi_{K M}}-\text {. }
$$

Proof. Proposition 3.3 of BBE02] verifies that the classical notion of the KacMoody extension of loop groups has this property. In BGW18b, §5.3, and Prop. $5.3]$ we compare the extension $\phi_{K M}$ with its classical definition in terms of determinant lines. 
5.3. Higher Contou-Carrère symbols. We begin this Subsection with a definition, in order to avoid the cumbersome notation $A\left(\left(t_{1}\right)\right) \cdots\left(\left(t_{n}\right)\right)^{\times}$.

Definition 5.7. The $n$-fold loop group $L^{n} G$ of a group-valued presheaf $G$ is defined to be the group-valued presheaf which sends the affine scheme $\operatorname{Spec} A$ to $G\left(A\left(\left(t_{1}\right)\right) \cdots\left(\left(t_{n}\right)\right)\right)$.

There is an analogue of the Kac-Moody extension for loop groups. Denoting by $\mathcal{V}_{A}^{n}$ the $n$-Tate object $A\left(\left(t_{1}\right)\right) \ldots\left(\left(t_{n}\right)\right)$ in $n$-Tate $\left(P_{f}(A)\right)$, we have a natural map

$$
L^{n} \mathbb{G}_{m}(A) \rightarrow \operatorname{Aut}\left(\mathcal{V}_{A}^{n}\right)
$$

for every $k$-algebra $A$. The index map gives rise to a spectral extension

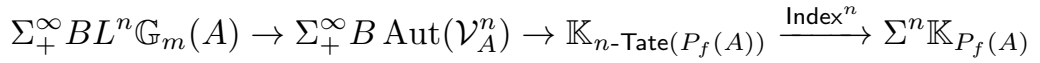

of $L^{n} \mathbb{G}_{m}(A)$ by $\Sigma^{n-2} \mathbb{K}_{P_{f}(A)}$. As above, the construction is natural in maps $A \rightarrow A^{\prime}$, so it defines a central extension of sheaves in groups.

Definition 5.8. The spectral extension (51) of $L^{n} \mathbb{G}_{m}$ by $\Sigma^{n-2} \mathbb{K}$ will be referred to as the canonical spectral extension of the $n$-fold loop group $L^{n} \mathbb{G}_{m}$. We denote the corresponding map of spectra by $e_{n}$.

As an application of this construction we give a definition of higher ContouCarrère symbols.

Definition 5.9. Let $f_{0}, \ldots, f_{n} \in L^{n} \mathbb{G}_{m}(A)=A\left(\left(t_{1}\right)\right) \ldots\left(\left(t_{n}\right)\right)^{\times}$. We denote by det the determinant map $K_{1}(A) \rightarrow A^{\times}$. The Contou-Carrère symbol $\left(f_{0}, \ldots, f_{n}\right)$ is defined to be the higher commutator

$$
\operatorname{det}\left(\left(f_{0} \star \cdots \star f_{n}\right)_{e_{n}}^{(-1)^{n}}\right) .
$$

The study of the higher Contou-Carrère symbol $\left(f_{0}, \ldots, f_{n}\right)$ for an $(n+1)$ tuple in $A\left(\left(t_{1}\right)\right) \ldots\left(\left(t_{n}\right)\right)$, with $A$ a $k$-algebra, has been pioneered by Osipov-Zhu in the case of $n=2$ (see OZ16]). They identified this symbol with a higher commutator in a central extension of the double loop group $L^{2} \mathbb{G}_{m}$ by $B^{\mathbb{Z}} \mathbb{G}_{m}$. Inspired by this observation and the one-dimensional case (Proposition [5.6), they define the two-dimensional Contou-Carrère symbol for general $k$-algebras $A$ as a higher commutator $C_{3}(f, g, h)$.

Proposition 5.10. Definition 5.9 is compatible with the definition of ContouCarrère in dimension 1, and Osipov-Zhu in dimension 2.

The proof of the 1-dimensional case was the content of Proposition 5.6. We now turn to verifying the assertion for $n=2$.

Proof of the 2-dimensional case. Osipov-Zhu construct a central extension of $L^{2} \mathbb{G}_{m}$ by the Picard groupoid $B^{\mathbb{Z}} \mathbb{G}_{m}$ ([OZ16, p. 28]), and define $(f, g, h)$ for a triple in $A\left(\left(t_{1}\right)\right)\left(\left(t_{2}\right)\right)^{\times}$, as the higher commutator $C_{3}(f, g, h)$. We have seen in Proposition 4.23 that $f \star g \star h=C_{3}(f, g, h)$. So to conclude the assertion, we need to verify that for $n=2$ the spectral extension of $L^{n} \mathbb{G}_{m}$ constructed in Definition 5.8 is related to the extension

$$
\Sigma_{+}^{\infty} B L^{2} \mathbb{G}_{m} \rightarrow \Sigma^{2} \mathbb{B}^{\mathbb{Z}} \mathbb{G}_{m}
$$

constructed by Osipov-Zhu. 
By Nisnevich descent, it suffices to consider rings $A$ with $K_{-1}(A)=0$. We then have a commutative diagram

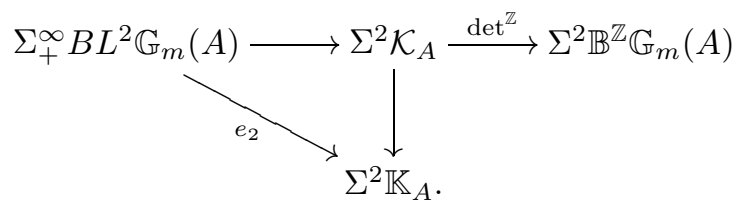

Using the adjunction between $\Sigma_{+}^{\infty}$ and $\Omega^{\infty}$, we obtain a map

$$
e_{2}: B L^{2} \mathbb{G}_{m} \rightarrow B^{2} B^{\mathbb{Z}} \mathbb{G}_{m}
$$

Picking a basepoint in $B L^{2} \mathbb{G}_{m}$ and looping once yields an $E_{1}$-map to the classifying space of the Picard groupoid of graded lines $B^{\mathbb{Z}} \mathbb{G}_{m}$

$$
\phi: L^{2} \mathbb{G}_{m} \rightarrow B B^{\mathbb{Z}} \mathbb{G}_{m} .
$$

We have to show that this morphism is -1 times of the one constructed by OsipovZhu. According to BGW18a, Prop. $3.28 \&$ Thm. 3.31], $\phi$ sends $f \in L^{2} \mathbb{G}_{m}(A)$ to

$$
\operatorname{det}^{\mathbb{Z}}(N / f L) \otimes \operatorname{det}^{\mathbb{Z}}(N / L)^{\vee},
$$

for $N$ a lattice containing both $f L$ and $L$, with the monoidal structure being defined in terms of common enveloping lattices. This is precisely the dual of the definition given by Osipov-Zhu [OZ16, p. 28].

The comparison of the generalized Contou-Carrère symbol with the classical cases in dimension 1 and 2 already shows that our definition produces a non-trivial map in these dimensions. We will explain why this is also the case in general.

Remark 5.11. Let $k \subset k^{\prime}$ be a field extension and $A \rightarrow^{\phi} k^{\prime}$ a ring homomorphism. Since our constructions are functorial in the $k$-algebra $A$ we see that

$$
\phi\left(\left(f_{0}, \ldots, f_{n}\right)\right)=\left(\phi\left(f_{0}\right), \ldots, \phi\left(f_{n}\right)\right)
$$

for $f_{0}, \ldots, f_{n} \in L^{n} \mathbb{G}_{m}(A)$. If we choose $f_{i}=t_{i+1}$ for $i=0, \ldots, n-1$ and $f_{n} \in k^{\times}$ we obtain $\phi\left(\left(f_{0}, \ldots, f_{n}\right)\right)=\phi\left(f_{n}\right)$. This follows from Corollary 6.6 below, which asserts that the higher Contou-Carrère symbol for $A$ a field agrees with the tame symbol.

After these preparations, let us return to geometry. Let $X$ be a Noetherian $k$ scheme and $\xi$ a saturated flag of integral closed subschemes $Z_{i}$. Moreover, suppose we are given a $k$-algebra $A$. Equipped with this data, we defined objects $F_{X, \xi}$ and $A_{X, \xi}$ in 3.1 .

By Theorem 7.10 of BGW16c, the object $F_{X, \xi}$ carries a canonical structure of a higher Tate object. In particular, we see that $F_{X, \xi}$ gives rise to an $n$-Tate object $\underline{F}_{X, \xi}$ in the abelian category $\operatorname{Coh}_{Z_{0}}(X)$ (coherent sheaves on $X$, set-theoretically supported at $Z_{0}$ ). If $X$ is defined over a field $k$, then, because $Z_{0}$ is 0 -dimensional, global sections give rise to an exact functor

$$
\Gamma(X,-): n \text {-Tate }\left(\operatorname{Coh}_{Z_{0}} X\right) \rightarrow n \text {-Tate }(k) .
$$

Thus, $F_{X, \xi}$ gives rise to an $n$-Tate object in the category of finite-dimensional vector spaces over $k$. If $A$ is an arbitrary $k$-algebra, the tensor product $-\otimes_{k} A$ : $\operatorname{Vect}_{f}(k) \rightarrow$ $P_{f}(A)$ determines an exact functor

$$
-\widehat{\otimes}_{k} A: n \text {-Tate }(k) \rightarrow n \text {-Tate }(A) .
$$


Definition 5.12. Let $X, \xi, k$, and $A$ be as described earlier. We define

$$
\underline{A}_{X, \xi}=F_{X, \xi} \widehat{\otimes}_{k} A .
$$

The $A$-module underlying $\underline{A}_{X, \xi}$ (via the forgetful functor $n$-Tate $(A) \rightarrow \operatorname{Mod}(A)$ ) inherits a $k$-algebra structure from $F_{X, \xi}$; we denote this $k$-algebra by $A_{X, \xi}$. For a group scheme $G$ over $k$, we define the iterated loop group at $(X, \xi)$ to be the group-valued presheaf given by

$$
L_{X, \xi}^{n} G(A)=G\left(A_{X, \xi}\right) .
$$

By definition, we have $L_{X, \xi}^{n} \mathbb{G}_{m}=F_{X, \xi}^{\times}$.

Example 5.13. If $X=\mathbb{A}_{k}^{n}=\operatorname{Spec} k\left[t_{1}, \ldots, t_{n}\right]$, and $Z_{k}=\operatorname{Spec} k\left[t_{1}, \ldots, t_{k}\right]$, then we have $A_{X, \xi}=A\left(\left(t_{1}\right)\right) \ldots\left(\left(t_{n}\right)\right)$, and $L_{X, \xi}^{n} \mathbb{G}_{m} \cong L^{n} \mathbb{G}_{m}$.

Note that for any ring $R$, the exact category of finitely-generated projective modules $P_{f}(R)$ is the idempotent completion of the exact category of finitely-generated free $R$-modules. Therefore, any exact functor $\phi: P_{f}(R) \rightarrow \mathrm{C}$, into any idempotent complete exact category $\mathrm{C}$, is determined by $\phi(R)$ up to equivalence.

Definition 5.14 (Spectral Contou-Carrère symbol). Let $T: P_{f}\left(A_{X, \xi}\right) \rightarrow n$-Tate $(A)$ be the unique functor sending $A_{X, \xi}$ to $\underline{A}_{X, \xi}$. The composition

$$
\sigma_{X, \xi}^{A}: \mathbb{K}_{A_{X, \xi}} \stackrel{T}{\rightarrow} \mathbb{K}_{n-\operatorname{Tate}(A)} \stackrel{(-1)^{n} \operatorname{Index}^{n}}{\longrightarrow} \Sigma^{n} \mathbb{K}_{A}
$$

will be referred to as the spectral Contou-Carrère symbol.

Replacing $K$-theory by $G$-theory (i.e. working with all coherent sheaves instead of only locally free ones), we obtain an analogous spectrification of the tame symbol.

Definition 5.15. Let $T: P_{f}\left(F_{X, \xi}\right) \rightarrow n$-Tate $\left(\mathrm{Coh}_{Z_{0}}(X)\right)$ be the unique functor sending $F_{X, \xi}$ to $\underline{F}_{X, \xi}$. The composition

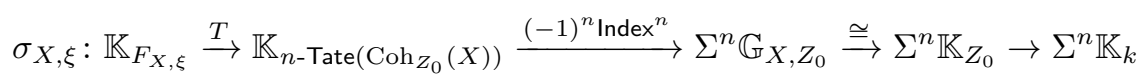

will be referred to as the spectral tame symbol.

Switching back to the Contou-Carrère setup, we can use higher commutators to extract Contou-Carrère symbols from the morphism of spectra in Definition 5.14 .

Definition 5.16. Denote by $\operatorname{det}: K_{1}(A) \rightarrow A^{\times}$the map induced by the determinant of matrices. For an $n+1$-tuple $f_{0}, \ldots, f_{n} \in A_{X, \xi}^{\times}$we define

$$
\left(f_{0}, \ldots, f_{n}\right)=\operatorname{det}\left(\left(f_{0} \star \cdots \star f_{n}\right)_{\sigma_{X, \xi}^{A}}\right),
$$

and refer to this expression as the Contou-Carrère symbol of $X$ at $\xi$.

\section{Comparison of Both DEFinitions}

This section is devoted to linking higher Contou-Carrère symbols to their classical counterparts. 


\section{1. $K$-theory and Tate categories.}

6.1.1. For exact categories. Schlichting developed a localization theorem for exact categories in [Sch04], which states that, for every left (respectively right) s-filtering inclusion of exact categories $\mathrm{C} \hookrightarrow \mathrm{D}$, the quotient category $\mathrm{D} / \mathrm{C}$ carries a natural structure as an exact category. Moreover, attaching their associated stable $\infty$ categories to them as in Definition 2.6.

$$
\operatorname{Perf}(\mathrm{C}) \stackrel{i}{\longrightarrow} \operatorname{Perf}(\mathrm{D}) \stackrel{q}{\longrightarrow} \operatorname{Perf}(\mathrm{D} / \mathrm{C})
$$

becomes an exact sequence of stable $\infty$-categories. Further, if $\mathrm{C}$ is idempotent complete, then by Proposition 2.4 (3), we obtain a bi-cartesian square of spectra

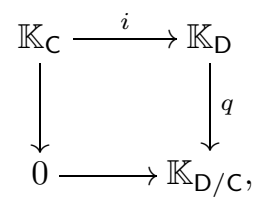

where the relation $\mathbb{K}_{C}=\mathbb{K}_{\text {Perf(C) }}$ holds essentially by definition. Schlichting observed that if one chooses $D$ such that $\mathbb{K}_{D} \cong 0$, then the boundary morphism of this square gives an equivalence $\partial: \mathbb{K}_{\mathrm{D} / \mathrm{C}} \cong \Sigma \mathbb{K}_{\mathrm{C}}$. Proposition 2.4 (1) guarantees that $\mathbb{K}_{\text {Ind }^{a}(\mathrm{C})} \cong 0$ for every exact category $\mathrm{C}$. Thus, we see that, for $\mathrm{C}$ idempotent complete, we have a canonical delooping $\mathbb{K}_{\text {Ind }^{a}(\mathrm{C}) / \mathrm{C}} \cong \Sigma \mathbb{K}_{\mathrm{C}}$. Using similar techniques, Saito establishes an abstract equivalence $\mathbb{K}_{\text {Tate(C) }} \cong \Sigma \mathbb{K}_{C}$ in [Sai15. In fact, this equivalence can be constructed as the composition

$$
\varphi: \mathbb{K}_{\text {Tate }(\mathrm{C})} \cong \mathbb{K}_{\operatorname{Tate}^{e l}(\mathrm{C})} \cong \mathbb{K}_{\operatorname{Tate}^{e l}(\mathrm{C}) / \operatorname{Pro}^{a}(\mathrm{C})} \cong \mathbb{K}_{\text {Ind }^{a}(\mathrm{C}) / \mathrm{C}}
$$

followed by

$$
\mathbb{K}_{\operatorname{Ind}^{a}(\mathrm{C}) / \mathrm{C}} \cong \Sigma \mathbb{K}_{\mathrm{C}}
$$

In the first row, the first equivalence follows from the cofinal invariance of nonconnective $K$-theory (i.e. (2) of Proposition 2.4). The second map is an equivalence as a corollary of the aforementioned localization theorem, and the third equivalence exists already on the level of exact categories (e.g. [BGW16c, Prop. 5.32]).

The index map of Definition 5.4 is an explicit description of these boundary maps. See [BGW18b, Thm. 3.6] for the proof.

Theorem 6.1. Let $\mathrm{C}$ be an idempotent complete exact category. The exact equivalence of exact categories $q: \operatorname{Tate}^{e l}(\mathrm{C}) / \operatorname{Pro}(\mathrm{C}) \cong \operatorname{Ind}^{a}(\mathrm{C}) / \mathrm{C}$ (see [BGW16c, Prop. 5.32]) induces a commutative diagram

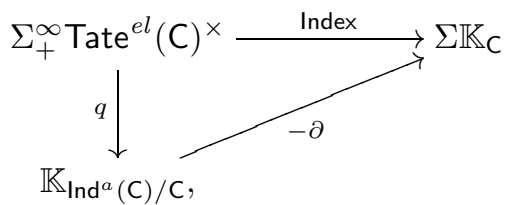

where both Index and $\partial$ arise as the boundary maps of the localization sequences discussed above.

This theorem motivates the following definition of the non-connective index map. 
Definition 6.2. For an idempotent complete exact category C, we define the map Index: $\mathbb{K}_{\text {Tate(C) }} \rightarrow \Sigma \mathbb{K}_{\mathrm{C}}$ as the composition so that the diagram

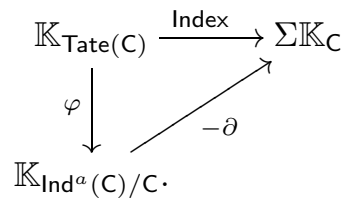

commutes, where $\varphi$ is the map of Equation (53).

6.1.2. Suspension and Calkin objects for stable $\infty$-categories. Let C be a stable $\infty$-category, and $\kappa$ an infinite cardinal. Recall Definition 2.5, which defines the suspension $\mathcal{S}_{\kappa}(\mathrm{C})$ as the localization

$$
\mathcal{S}_{\kappa}(\mathrm{C})=\operatorname{Ind}_{\kappa}(\mathrm{C}) / \mathrm{C},
$$

and which defines the $\infty$-category of Calkin objects Calk(C) as the idempotent completion of the suspension.

Since non-connective $K$-theory cannot distinguish between a category and its idempotent completion (see (2) of Proposition 2.4): $\mathbb{K}_{\text {Calk }_{\kappa}(\mathrm{C})} \cong \mathbb{K}_{\mathcal{S}_{\kappa}(\mathrm{C})} \cong \Sigma \mathbb{K}_{\mathrm{C}}$, we will often omit the cardinal $\kappa$ from our notation. Following Schlichting [Sch04, Blumberg-Gepner-Tabuada BGT13] observed the following delooping property for $K$-theory introduced in (32).

Proposition 6.3. The boundary map $\partial$ of the localization sequence of the exact sequence

$$
\mathrm{C} \hookrightarrow \operatorname{Ind}(\mathrm{C}) \rightarrow \mathcal{S}(\mathrm{C})
$$

of stable $\infty$-categories, induces an equivalence of non-connective $K$-theory spectra $\partial: \mathbb{K}_{\text {Calk (C) }} \cong \Sigma \mathbb{K}_{\mathrm{C}}$.

This result serves as a motivation to call $\mathcal{S}(\mathrm{C})$ the suspension of $\mathrm{C}$. Recall that the suspension of a topological space $X$ is formed by embedding $X$ into the cone $C X$, which is contractible. The resulting homotopy cofibre, obtained by taking the quotient space, is one possible incarnation of the suspension. By analogy, Schlichting embeds a category $\mathrm{C}$ into an ambient $K$-contractible category $\operatorname{lnd}(\mathrm{C})$, and takes the quotient to obtain the categorical suspension. A second possibility is to construct the suspension of $X$ by glueing a second copy $C^{-} X$ to the cone $C X$ along the common subspace $X$. Since $C^{-} X$ is contractible, this yields a homotopy equivalent space. Categorically this is analogous to pasting the $K$-contractible categories Ind $^{a}(\mathrm{C})$ and $\operatorname{Pro}^{a}(\mathrm{C})$ along the common subcategory C. This is the underlying idea of Saito's delooping statement. For later use, we record the following naturality property.

Lemma 6.4 (Naturality). For every idempotent complete exact category $\mathrm{C}$, there exists a commutative diagram

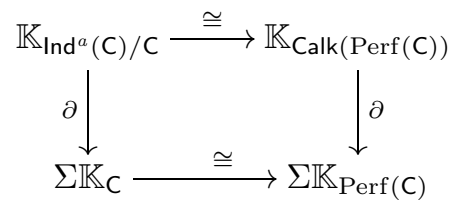


of spectra, where the horizontal maps $\mathbb{K}_{\mathrm{C}} \cong \mathbb{K}_{\mathrm{Perf}(\mathrm{C})}$ are the equivalences stipulated by Lemma 2.7, and the vertical maps are the equivalences coming from the boundary maps of the localization sequence discussed above.

\subsection{Comparison.}

Theorem 6.5. Let $X$ be a Noetherian $k$-scheme, and $\xi$ a saturated flag of closed subschemes $Z_{i}$. For every $k$-algebra $A$, the spectral Contou-Carrère symbol $\sigma_{X, \xi}^{A}$ of Definition 5.14 agrees with the $n$-fold delooping of the preliminary Contou-Carrère symbol of Definition 3.16 .

The proof of this result will be given in the next paragraph. It uses the concept of realization functors which we will subsequently introduce. We conclude:

Corollary 6.6. For A a field, the Contou-Carrère symbol agrees with the higher tame symbol for algebraic $K$-theory.

\subsection{Contou-Carrère symbols and realization functors.}

Definition 6.7 (Tate realization). Let $X$ be a Noetherian scheme, and $j: U \hookrightarrow X$ an open immersion, with complement denoted by $Z$. Let $W \supset Z$ be a closed subscheme of $X$, such that the open immersion $U \cap W \hookrightarrow W$ is affine 26 Then, we have exact functors ind, pro, and tate, defined as follows.

(a) The functor ind: $\operatorname{Coh}_{|W| \cap U}(U) \rightarrow \operatorname{Ind}^{a}\left(\operatorname{Coh}_{|W|}(X)\right)$ sends $\mathcal{F} \in \operatorname{Coh}_{|W| \cap U}(U)$ to $j_{*} \mathcal{F}$, viewed as an ascending union of coherent sheaves on $X$ with settheoretic support in $|W|$.

(b) We denote by $i_{n}: Z^{(n)} \rightarrow X$ the inclusion of the $n$-th order infinitesimal neighbourhood of $Z$. We define pro: $\operatorname{Coh}_{W}(X) \rightarrow \operatorname{Pro}^{a}\left(\mathrm{Coh}_{|Z|}(X)\right)$ to be the functor sending $\mathcal{F} \in \mathrm{Coh}_{|W|}(X)$ to the Pro-system $\left(\left(i_{n}\right)_{*} i_{n}^{*} \mathcal{F}\right)_{n \in \mathbb{N}}$.

(c) Combining (a) and (b) we obtain a functor

$$
\text { tate: } \operatorname{Coh}_{|W|}(U) \rightarrow \operatorname{Ind}^{a} \operatorname{Pro}^{a}\left(\operatorname{Coh}_{|Z|}(X)\right) \text {. }
$$

Remark 6.8. One can check that the functor tate of Definition 6.7(c) factors through $\operatorname{Tate}^{e l}\left(\operatorname{Coh}_{Z}(X)\right) \subset$ Ind $^{a} \operatorname{Pro}^{a}\left(\operatorname{Coh}_{Z}(X)\right)$. Indeed, for every $\mathcal{F} \in \operatorname{Coh}_{|W| \cap U}(U)$ we have a 4 -term exact sequence

$$
\operatorname{ker}(\operatorname{pro}(\mathcal{F}) \rightarrow \operatorname{tate}(\mathcal{F})) \hookrightarrow \operatorname{pro}(\mathcal{F}) \rightarrow \operatorname{tate}(\mathcal{F}) \rightarrow j_{*} j^{*} \mathcal{F} / \mathcal{F} .
$$

The kernel on the left hand side is equivalent to $\operatorname{ker}\left(\mathcal{F} \rightarrow j_{*} j^{*} \mathcal{F}\right) \in \operatorname{Coh}_{Z}(X)$, hence the quotient of $\operatorname{pro}(\mathcal{F})$ by this object lies again in $\operatorname{Pro}^{a}\left(\operatorname{Coh}_{Z}(X)\right)$. The object on the right hand side $j_{*} j^{*} \mathcal{F} / \mathcal{F}$ lies in $\operatorname{Ind}^{a}\left(\operatorname{Coh}_{Z}(X)\right)$. This allows us to represent tate $(\mathcal{F})$ as an extension of an admissible Ind-object by an admissible Pro object. Hence, $\operatorname{tate}(\mathcal{F})$ has a lattice, i.e. is an elementary Tate object.

Definition 6.7 contains the condition that the inclusion $U \cap W \hookrightarrow W$ is affine. It is important to note the following two observations.

Lemma 6.9. We have the following:

(a) Let $W, W^{\prime} \hookrightarrow X$ be closed subschemes of a separated Noetherian scheme $X$, satisfying $|W|=\left|W^{\prime}\right|$. For an open subscheme $U \subset X$ we have that $W \cap U \hookrightarrow W$ is affine if and only if $W^{\prime} \cap U \hookrightarrow W^{\prime}$ is affine.

\footnotetext{
${ }^{26}$ In Lemma 6.9 (a) we show that this is condition only depends on the underlying closed subspace $|W|$.
} 
(b) Let $W \hookrightarrow X$ be a closed immersion into a separated Noetherian scheme $X$, and $U \subset X$ an open subscheme with closed complement denoted by $|Z|$. If $|Z| \subset|W|$, and $\operatorname{dim} Z=\operatorname{dim} W-1=0$, then the inclusion $W \cap U \hookrightarrow W$ is affine.

Proof. Assertion (a) follows from the fact that a scheme is affine if and only if the underlying reduced scheme is affine (which is a consequence of Chevalley's theorem, see [Con07]). To verify (b), we observe that $Z=\left\{z_{1}, \ldots, z_{k}\right\}$ is a discrete subset consisting of closed points (since it is of dimension 0 ), and we may therefore replace $W$ without loss of generality by $\operatorname{Spec}\left(\mathcal{O}_{W, z_{1}} \times \cdots \times \mathcal{O}_{W, z_{k}}\right)$. Then, the complement $W \cap U=W \backslash Z$ agrees with the discrete subset $\left\{\eta_{1}, \ldots, \eta_{m}\right\}$, where each $\eta_{i}$ is a generic point of the one-dimensional scheme $W$. Since each of the inclusions $\left\{\eta_{i}\right\} \hookrightarrow X$ is affine, the same is true for

$$
W \cap U=\coprod_{i=1}^{m}\left\{\eta_{i}\right\} \hookrightarrow X,
$$

since a finite coproduct of affine schemes is affine.

We call these functors realization functors, since they associate to a coherent sheaf on $U=X \backslash Z$ a Tate object in $\operatorname{Coh}_{Z}(X)$. For our purposes it will be necessary to have similar functors for perfect complexes on $U$ at our disposal. This is achieved by the following definition. We denote the derived $\infty$-category of pseudo-coherent complexes of sheaves (resp. complexes of quasi-coherent sheaves) on a scheme $X$ by $\mathrm{DCoh}(X)$ (resp. DQCoh $(X))$.

Definition 6.10 (Calkin realization). Let $X, U, Z$ be quasi-compact and quasiseparated schemes, with $j: Z \hookrightarrow X$ a closed embedding, and $U=X \backslash Z$.

(a) We denote by

$$
\text { ind: } \operatorname{Perf}(X) \rightarrow \operatorname{Ind}\left(\operatorname{Perf}_{Z}(X)\right) \cong \mathrm{DQCoh}_{Z}(X)
$$

the functor given by $\mathcal{F} \mapsto \operatorname{fib}\left(\mathcal{F} \rightarrow j_{*} j^{*} \mathcal{F}\right)$.

(b) Let calk: $\operatorname{Perf}(U) \rightarrow \operatorname{Calk}\left(\operatorname{Perf}_{Z}(X)\right)$ be the functor induced by ind:

$$
\operatorname{Perf}(U) \simeq\left(\operatorname{Perf}(X) / \operatorname{Perf}_{Z}(X)\right)^{\text {ic }} \rightarrow\left(\operatorname{Ind}\left(\operatorname{Perf}_{Z}(X)\right) / \operatorname{Perf}_{Z}(X)\right)^{\text {ic }} .
$$

(c) The functors ind and calk have a version for the stable $\infty$-categories of pseudo-coherent complexes of sheaves:

$$
\text { ind: } \operatorname{DCoh}(X) \rightarrow \operatorname{lnd}\left(\operatorname{DCoh}_{Z}(X)\right),
$$

and

$$
\text { calk: } \mathrm{DCoh}(U) \rightarrow \operatorname{Calk}_{\left(\mathrm{DCoh}_{Z}(X)\right) .}
$$

Lemma 6.11. Let $X, U$, and $j: Z \hookrightarrow X$ be as in Definition 6.10. Let $X \rightarrow W$ be a morphism of schemes. For an affine flat morphism $f: V \rightarrow W$ we denote the base changes $X \times_{W} V, U \times_{W} V$, and $Z \times_{W} V$ by $X_{V}, U_{V}$, and $Z_{V}$. We then have a commutative diagram

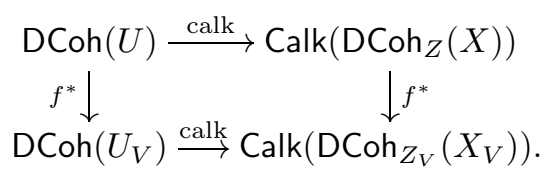


Proof. The assumptions on $f$ imply that we have a commuting square

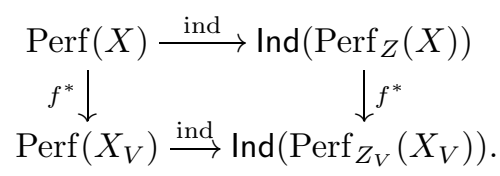

By quotienting and taking idempotent, we obtain the commuting square of the lemma.

The Tate and Calkin realization for coherent sheaves (respectively pseudo-coherent complexes) are related by the composition of the natural exact functors

$$
\operatorname{Tate}\left(\mathrm{Coh}_{Z}(X)\right) \stackrel{q}{\rightarrow} \operatorname{Calk}\left(\mathrm{Coh}_{Z}(X)\right) \stackrel{\Psi}{\rightarrow} \operatorname{Calk}\left(\mathrm{DCoh}_{Z}(X)\right) .
$$

Lemma 6.12. Let $X$ be Noetherian, and $Z, U$, and $W$ satisfy the conditions of Definition 6.7. We have a commuting square

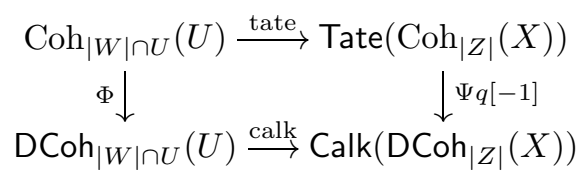

of $\infty$-categories, where $\Phi: \operatorname{Coh}_{|W| \cap U}(U) \rightarrow \mathrm{DCoh}_{|W| \cap U}(U)$ denotes the canonical functor.

Proof. According to Definition 6.10 we have that, for every pseudo-coherent complex $\mathcal{F}$ on $X$ with set-theoretic support in $|W|$,

$$
\operatorname{calk}\left(j^{*} \mathcal{F}\right)=\operatorname{fib}\left(\mathcal{F} \rightarrow j_{*} j^{*} \mathcal{F}\right) .
$$

Since $j$ is proper and affine by assumption, we have that for $\mathcal{F} \in \operatorname{Coh}(X)$ the expression $j_{*} j^{*} \mathcal{F}$ has vanishing higher cohomology groups. In particular, $\operatorname{calk}\left(j^{*} \mathcal{F}\right)$ can be represented by the admissible Ind-object $j_{*} j^{*} \mathcal{F} / \mathcal{F}[-1]$. By Remark 6.8, this admissible Ind-object represents the Calkin object corresponding to the Tate object tate $(\mathcal{F})$. The general case, i.e. of a coherent sheaf on $U$ which does not extend to $X$, follows by passing to idempotent completions.

The discussion above gives rise to the top square in the commutative diagram below, where $\Phi: \operatorname{Coh}_{|W| \cap U}(U) \rightarrow \operatorname{DCoh}_{|W| \cap U}(U)$ denotes the canonical functor to the derived category, and $q$ is the exact functor of Theorem 6.1.

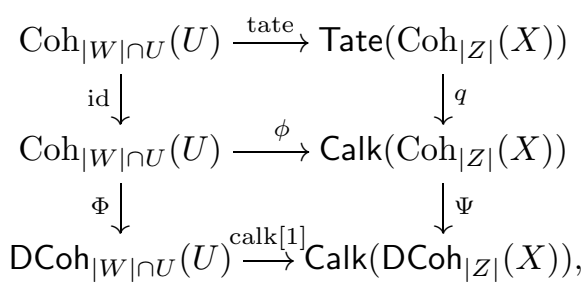

where $\phi$ is the functor obtained by sending $\mathcal{F} \in \operatorname{Coh}_{|W| \cap U}(U)$ to $j_{*} \mathcal{F} / \widetilde{F}$ $\in \operatorname{Calk}\left(\operatorname{DCoh}_{|Z|}(X)\right)$, where $\widetilde{\mathcal{F}}$ is a pseudo-coherent subsheaf of $j_{*} \mathcal{F}$, such that $j^{*} \widetilde{F}=\mathcal{F}$. The outer square yields the required commutative diagram. 
For $X$ a Noetherian scheme, and $\xi$ a saturated flag of closed subschemes we denote by $X^{[m]}$ the scheme obtained by applying Definition 3.12 of $X^{(m)}$ for $A=k$. We now construct a sequence of Tate realization functors

$$
\text { tate: } \operatorname{Coh}_{Z_{j}^{(j)}}\left(X^{[j]}\right) \rightarrow \operatorname{Tate}\left(\operatorname{Coh}_{Z_{j-1}^{[j-1]}}\left(X^{[j-1]}\right)\right) .
$$

Lemma 6.9 (b) implies that the crucial affineness condition of Definition 6.7 is satisfied in this now case for dimension reasons. Composition of these exact functors yields a well-defined exact functor

$$
\operatorname{tate}^{n}: \operatorname{Coh}\left(X^{[n]}\right) \rightarrow n \text {-Tate }\left(\operatorname{Coh}_{Z_{0}}(X)\right) .
$$

The proposition below can also be obtained from BGW16c.

Proposition 6.13. The functor $\pi_{*}$ tate $^{n}$ agrees with the ( $n$-Tate object valued) Beilinson-Parshin adèles $\underline{F}_{X, \xi}$.

Proof. The functors ind and pro of Definition 6.7 mirror localization and completion with respect to the scheme $X$. In particular, we see for $\mathcal{F} \in \operatorname{Coh}(W)$ that $\pi_{*} \operatorname{tate}^{n}(\mathcal{F})=\underline{F}_{X, \xi}$.

Composing $n$-Tate with pushforward $\pi: \operatorname{Coh}_{Z_{0}}(X) \rightarrow \operatorname{Coh}(\operatorname{Spec} k)=\operatorname{Vect}_{f}(k)$, we obtain an exact functor $\operatorname{Coh}\left(X^{[n]}\right) \rightarrow n$-Tate $(k)$.

Definition 6.14. Let $X$ be a Noetherian $k$-scheme, and $\xi$ a saturated flag of closed subschemes. For every $k$-algebra $A$ we denote by $\operatorname{Coh}^{b}\left(X^{(n)}\right)$ the full subcategory of $\operatorname{Coh}\left(X^{(n)}\right)$, consisting of coherent sheaves which are pulled back along the canonical map $s: X^{(n)} \rightarrow X^{[n]}$. Denoting by

$$
(-)_{A}: n \text {-Tate }(k) \rightarrow n \text {-Tate }(A)
$$

the exact functor induced by $-\otimes_{k} A: \operatorname{Vect}_{f}(k) \rightarrow P_{f}(A)$, we have a unique $A$-linear functor

$$
\left(\pi_{*} \circ \operatorname{tate}^{n}\right)_{A}: \operatorname{Coh}^{b}\left(X^{(n)}\right) \rightarrow n \text {-Tate }(A),
$$

such that the following diagram commutes:

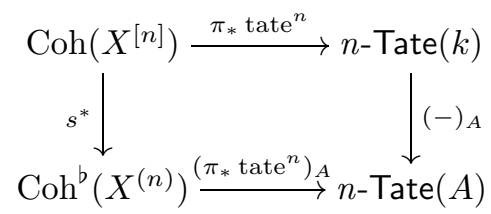

Proposition 6.15. We denote by $\mathrm{VB}_{f}(W)$ the exact category of free vector bundles on a scheme $W$. Let $X$ be a finite type, separated $k$-scheme of dimension $n$, and let $\xi$ be a saturated flag of closed subschemes. For every $k$-algebra $A$, the diagram

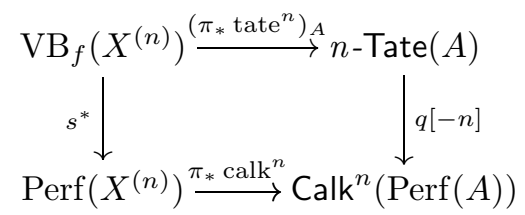

is commutative. 
Proof. For $A=k$ this follows from applying the comparison of Lemma 6.12 iteratively. The general case follows from the base change invariance of the Calkin realization (Lemma 6.11), and Definition 6.14 of the functor $\left(\pi_{*} \circ \operatorname{tate}^{n}\right)_{A}$ by base change.

We are now ready to prove that the spectral Contou-Carrère symbol $\sigma_{X, \xi}^{A}$ can be represented as the composition $\pi_{*} \circ \partial_{\mathcal{Z}_{X_{A}, \xi_{A}}^{\text {loc }}}$.

Proof of Theorem 6.5. Proposition 6.15 established a compatibility between the Tate and Calkin realization: $\pi_{*}$ calk $^{n} \simeq q\left(\pi_{*} \text { tate }^{n}\right)_{A}[-n]$. Applying the non-connective algebraic $K$-theory functor $\mathbb{K}_{-}$to this equivalence, we obtain two equivalent maps

$$
\mathbb{K}_{\pi_{*} \operatorname{calk}^{n}} \simeq \mathbb{K}_{q\left(\pi_{*} \operatorname{tate}^{n}\right)_{A}[-n]}: \mathbb{K}_{X^{(n)}} \rightarrow \mathbb{K}_{\text {Calk }^{n}(\operatorname{Perf}(A))} \simeq \Sigma^{n} \mathbb{K}_{A} .
$$

Here, we made use of the fact that non-connective algebraic $K$-theory is cofinally invariant, i.e. cannot distinguish between an exact category and its idempotent completion. In particular,

$$
\mathbb{K}_{\mathrm{VB}_{f}\left(X^{(n)}\right)} \cong \mathbb{K}_{\mathrm{VB}\left(X^{(n)}\right)} \cong \mathbb{K}_{X^{(n)}}
$$

By Proposition 6.13, and by the definition of $\left(\pi_{*} \text { tate }^{n}\right)_{A}$ by base change (Definition

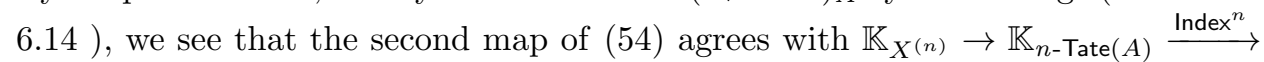
$\Sigma^{n} \mathbb{K}_{A}$. Hence, this map agrees with the spectral Contou-Carrère symbol, by Definition 5.14. To conclude the proof we have to compare the first map with the $n$-fold

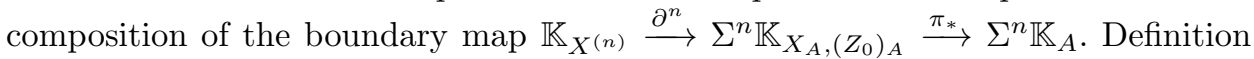
6.10 implies that for every triple $(X, U, Z)$ we have a commutative cube of stable $\infty$-categories

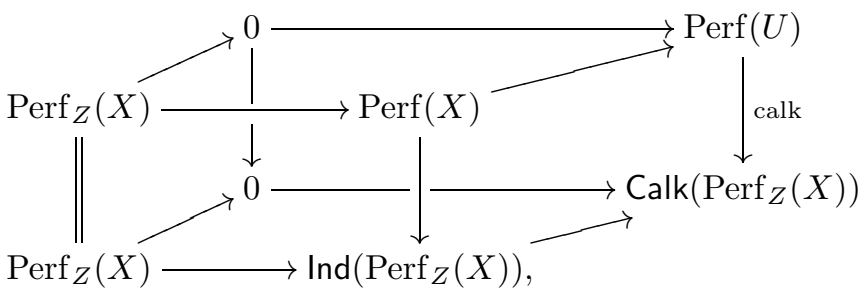

where the top square comes from the localization sequence of the closed embedding, and the lower square corresponds to the short exact sequence of stable $\infty$-categories

$$
\operatorname{Perf}_{Z}(X) \rightarrow \operatorname{Ind}\left(\operatorname{Perf}_{Z}(X)\right) \rightarrow \operatorname{Calk}\left(\operatorname{Perf}_{Z}(X)\right) .
$$

Since the top and bottom face are localization sequences, applying $\mathbb{K}_{-}$yields a commutative cube with top and bottom face being bi-cartesian. In particular, we obtain a commutative triangle relating the boundary maps of the bottom and top face:

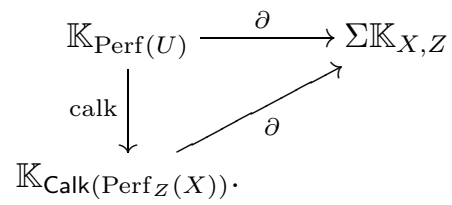

Applying this comparison $n$ times, we see that $\mathbb{K}_{\pi_{*} \text { calk }}$ is equivalent to $\pi_{*} \partial_{n} \circ \cdots \circ$ $\partial_{1}$. 


\section{RECIPROCITY}

Let $X$ be a proper integral curve over a field $k$. We write $X_{0}$ for its set of closed points. For every commutative $k$-algebra $A, x \in X_{0}$, and a pair of units in the ring of $A$-valued rational functions

$$
f, g \in A(X)^{\times}=\left(k(X) \otimes_{k} A\right)^{\times}
$$

the Contou-Carrère symbol gives an element $(f, g)_{x}$ of $A^{\times}$.

Theorem 7.1 (Weil, Anderson-Pablos Romo, Beilinson-Bloch-Esnault). The product below is well-defined and satisfies

$$
\prod_{x \in X_{0}}(f, g)_{x}=1
$$

This reciprocity law has been proven by Weil for $A=k$, it was generalized to the case of artinian rings $A$ by Anderson-Pablos Romo [APR04, and to general $A$ by Beilinson-Bloch-Esnault [BBE02, §3.4]. Recently, Pál has shown in [Pál10] that, for artinian rings, the relative version of Weil reciprocity follows from the absolute case $(A=k)$ after a change of fields.

This section is concerned with an extension of this result to varieties of arbitrary dimension (and arbitrary rings $A$ ). The absolute case $(A=k)$ is due to Kato Kat86] (however, the case of surfaces was pioneered by Parshin). Recent work of Osipov-Zhu OZ16 established a Contou-Carrère reciprocity law for surfaces and artinian rings.

Fix an integer $0 \leq i \leq n$. As before, $A$ denotes a $k$-algebra over a field $k$. The main player is an $n$-dimensional, integral, separated $k$-scheme of finite type $X$, together with an almost saturated flag

$$
\zeta=\left(X=Z_{n} \supset \cdots Z_{i+1} \supset Z_{i-1} \supset \cdots Z_{0}\right),
$$

of closed integral subvarieties, satisfying $\operatorname{dim} Z_{j}=j$. If $i=0$, we assume that $Z_{1}$ is proper over $k$.

For every closed equiheighted $i$-dimensional subset $Z$, satisfying $Z_{i+1} \supset Z \supset$ $Z_{i-1}$ we obtain a saturated flag $\xi_{Z}$. Note that we denote saturated flags by the letter $\xi$ for the sake of visual distinctness.

In order to formulate the reciprocity law, we need to construct an analogue of the ring of $A$-valued rational functions $A(X)$ on a curve $X$. This ring $A_{\zeta}(X)$ should be naturally associated to the data $(X, \zeta)$ and the $k$-algebra $A$. Further, for each $Z$ as above, we require a specialization homomorphism

$$
A_{\zeta}(X) \stackrel{i_{\zeta}}{\longrightarrow} A_{X, \xi_{Z}}
$$

The latter is required to make sense of the factors of the product

$$
\prod_{Z_{i+1} \supset Z \supset Z_{i-1}}\left(f_{0}, \ldots, f_{n}\right)_{\xi Z} .
$$

Definition 7.2. We define the following:

(a) Let $X$ be a separated $n$-dimensional $k$-scheme of finite type, $A$ a $k$-algebra, and $\zeta$ an almost complete flag in $X$. For each $Z_{i+1} \supset Z \supset Z_{i-1}$ with $Z$ of pure dimension $i$ and not necessarily irreducible, we denote the ring of 
regular functions on the scheme $(\mathrm{C} \circ \mathrm{L})^{n-i-1} \circ \mathrm{L} \circ(\mathrm{C} \circ \mathrm{L})^{i}\left(X_{A},\left(\xi_{Z}\right)_{A}\right)$ by $A_{\zeta, Z}(X)$. We define the ring $A_{\zeta}(X)$ to be the direct limit

$$
A_{\zeta}(X)=\lim _{Z_{i-1} \subset Z \subset Z_{i+1}} A_{\zeta, Z}(X)
$$

where $Z$ is a closed subset of pure dimension $i$ (not necessarily irreducible).

(b) For every $Z$ as in (a), we denote the natural ring homomorphism $A_{\zeta}(X) \rightarrow$ $A_{X, \xi_{Z}}$ by $i_{\zeta}$.

In the definition above we can apply the operations $\mathrm{L}$ and $\mathrm{C}$ because we may replace the scheme by a suitable affine open neighbourhood.

After having introduced this colimit, we observe that the algebraic $K$-theory is manageable for formal reasons. This will be used in the proof of our main result.

Remark 7.3. Since non-connective $K$-theory of rings commutes with filtered colimits (Theorem 7.2 of TT90) one has

$$
\mathbb{K}_{A_{X, \zeta}}=\lim _{Z_{i-1}} \underset{\subset Z \subset Z_{i+1}}{ } \mathbb{K}_{(\mathrm{CoL})^{n-i-1} \circ \mathrm{L} \circ(\mathrm{CoL})^{i}\left(X_{A},\left(\xi_{Z}\right)_{A}\right)} .
$$

We are now ready to state the main result of this section, in a classical formulation:

Theorem 7.4 (Reciprocity for Contou-Carrère symbols). Let $X$ be an integral separated $n$-dimensional $k$-scheme of finite type, and let $A$ be a commutative $k$ algebra. Let $\zeta$ be an almost saturated flag as in (55). For every $(n+1)$-tuple $f_{0}, \ldots, f_{n} \in A_{\zeta}(X)^{\times}$we have that the product below is well-defined and satisfies the identity

$$
\prod_{Z_{i+1} \supset Z \supset Z_{i-1}}\left(f_{0}, \ldots, f_{n}\right)_{\xi_{Z}}=1
$$

where $Z$ is integral and of dimension $i$.

We will deduce this result in Subsection 7.2 from an abstract reciprocity law for compositions of boundary maps (see Corollary 7.11). The reciprocity relation will be generalized to the existence of a null-homotopy for a certain map of spectra. We refer to such a construction as spectrification (following Beilinson).

\subsection{Abstract reciprocity laws.}

7.1.1. Notation. In Appendix B.1 we explain a mild generalization of a construction due to Efimov, which allows one to complete a stable $\infty$-category $C$ at a full subcategory $\mathbf{S}$. The resulting category is denoted by $\mathbf{C}_{\widehat{\mathbf{s}}}$. This is a categorical analogue of completion in commutative algebra. We refer the reader to the appendix of Efimov's Efi10 for more details.

Definition 7.5. Let $C$ be a stable $\infty$-category as in Paragraph B.2.1

(a) A chain of localizing subcategories $\mathbf{S}_{0} \subset \mathbf{S}_{1} \subset \ldots$, will be referred to as a flag in C.

(b) We denote $\mathbf{S}_{i}$ by $\mathrm{C}_{[i]}$.

(c) We denote $\mathrm{C}_{\widehat{\mathbf{S}_{i}}}$ by $\mathrm{C}_{\widehat{[i]}}$.

(d) We write $\mathrm{C}_{(i)}=\left(\mathrm{C} / \mathbf{S}_{i}\right)^{\text {ic }}$.

(e) We write $\mathrm{C}_{\widehat{(i)}}=\left(\mathrm{C}_{\widehat{\left[\mathbf{S}_{i}\right]}} / \mathbf{S}_{i}\right)^{\text {ic }}$. 
(f) Given a flag on $\mathrm{C}$ as above, we define the iterated removal-completion operation by

$$
\mathrm{C}_{\widehat{(0, n)}}=\left(\left(\mathrm{C}_{(\widehat{0, n-1})}\right)_{\widehat{\mathbf{S}_{n}}} /\left(\mathbf{S}_{n}\right)_{(\widehat{0, n-1})}\right)^{\mathrm{ic}},
$$

with $\mathrm{C}_{\emptyset}=\mathrm{C}$.

Let $X$ be a scheme. Given a flag of closed subschemes in $X$,

$$
\xi=\left(X=Z_{n} \supset Z_{n-1} \supset \cdots \supset Z_{0}\right),
$$

we obtain a flag of localizing subcategories $\mathbf{S}_{0}, \ldots, \mathbf{S}_{n-1}$ of $\operatorname{Perf}(X)$ by defining $\mathbf{S}_{i}$ to be $\operatorname{Perf}_{Z_{i}}(X)$. The following example is a special case of Proposition B.8 in the appendix.

Example 7.6. Let $X$ be affine $n$-space $\mathbb{A}^{n}=\operatorname{Spec} k\left[t_{1}, \ldots, t_{n}\right]$, and $\xi$ the flag given by $Z_{i}=\operatorname{Spec} k\left[t_{1}, \ldots, t_{i}\right]$. We then have $\mathrm{C}_{\widehat{(0, n)}} \cong \operatorname{Perf}\left(\operatorname{Spec} k\left(\left(t_{1}\right)\right) \ldots\left(\left(t_{n}\right)\right)\right)$.

7.1.2. Reciprocity laws. In the following we denote by $\mathrm{C}$ a stable $\infty$-category, and consider a chain $\mathbf{S}_{0}, \mathbf{S}_{1}, \ldots, \mathbf{S}_{n}$ of localizing subcategories (as considered above). We will be concerned with the composition of boundary maps, connecting the nonconnective $K$-theory spectra of various stable $\infty$-categories constructed from $C$ with the help of the localizing subcategories.

Let $\mathbf{C}$ be a stable $\infty$-category together with a localizing subcategory $\mathbf{S}$. With respect to the terminology introduced in Definition B.5 we have short exact sequences of stable $\infty$-categories

$$
\mathbf{S} \hookrightarrow \mathrm{C} \rightarrow \mathrm{C}_{(\mathbf{S})} \text { and } \mathbf{S} \hookrightarrow \mathrm{C}_{\widehat{\mathbf{S}}} \rightarrow \mathrm{C}_{\widehat{(\mathbf{S})}}
$$

and canonical functors

$$
\mathrm{C} \stackrel{F}{\rightarrow} \mathrm{C}_{\widehat{\mathrm{S}}} \text { and } \mathrm{C}_{(\mathbf{S})} \stackrel{G}{\rightarrow} \mathrm{C}_{\widehat{(\mathbf{S})}} .
$$

Furthermore, these short exact sequences and functors belong to a commutative diagram:

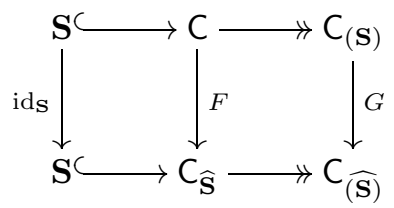

On the level of algebraic $K$-theory, the localization sequences yields a boundary map

$$
\Omega \mathbb{K}_{(\widehat{\mathbf{S}})} \stackrel{\widehat{\partial}}{\rightarrow} \mathbb{K}_{\mathbf{S}} .
$$

Theorem 7.7 (Abstract Weil reciprocity). Let $\mathrm{C}$ be a stable $\infty$-category together with a localizing subcategory $\mathbf{S}$. We assume the existence of an exact functor $\mathrm{C} \stackrel{c}{\rightarrow} \mathrm{D}$, where $\mathrm{D}$ denotes as well a stable $\infty$-category. We denote the inclusion $\mathbf{S} \hookrightarrow \mathrm{C}$ by $a$, and the restriction $\left.c\right|_{\mathbf{S}}$ by $b$ :

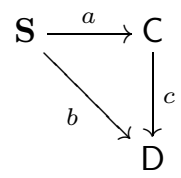


Under these assumptions the map $\Omega \mathbb{K}_{\mathrm{C}_{(\mathbf{S})}} \rightarrow \mathbb{K}_{\mathrm{D}}$ defined as the composition $b \circ \partial \circ G$

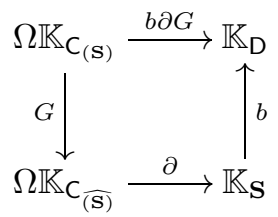

is homotopic to the zero map.

Proof. We have a commutative diagram:

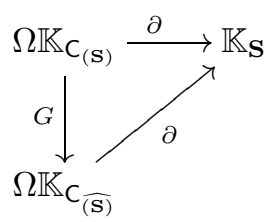

Commutativity of the diagram above follows from the naturality of boundary maps in algebraic $K$-theory (applied to (57)). This implies

$$
b \circ \partial \circ G \simeq c \circ a \circ \partial: \Omega \mathbb{K}_{(\mathbf{S})} \rightarrow \mathbb{K}_{\mathrm{D}} .
$$

We may therefore focus on establishing the null-homotopy of the map $a \circ \partial$. We have a commuting diagram of spectra, with the square being bi-cartesian:

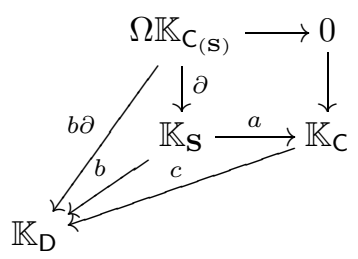

Commutativity of the square implies $a \circ \partial \simeq 0$.

Example 7.8 (Weil reciprocity). Let $X$ be a proper, integral curve over a field $k$, we set $\mathrm{C}=\operatorname{Perf}(X)$, and for every reduced 0-dimensional closed subscheme $Z$ (not assumed to be irreducible) we let $\mathbf{S}$ be $\operatorname{Perf}_{|Z|}(X)$. We then have $\mathbf{C}_{(\mathbf{S})} \cong \operatorname{Perf}(X \backslash Z)$. Using the (derived) pushforward functor to the base field $\pi_{*}$ : $\operatorname{Perf}(X) \rightarrow \operatorname{Perf}(k)$, Theorem 7.7 implies now that the canonical map

$$
\pi_{*} \partial: \Omega \mathbb{K}_{X \backslash Z} \rightarrow \mathbb{K}_{k}
$$

is homotopic to zero. The field of rational functions $k(X)$ arises as the direct limit $k(X) \cong \lim _{Z} \mathcal{O}_{X \backslash Z}$, in particular we have $\mathbb{K}_{k(X)} \cong \lim _{Z} \mathbb{K}_{X \backslash Z}$, by virtue of Theorem 7.2 in [TT90]. Since we have a functor from the direct limit of $\infty$ categories $\operatorname{Perf}(X)_{\widehat{(\mathbf{s})}}$ to $\operatorname{Perf}\left(\mathbb{A}_{X}\right)$, by virtue of Theorem $\mathbb{B . 1 1}(\mathrm{a})$, we obtain the commutative diagram in the stable $\infty$-category of spectra on the left:
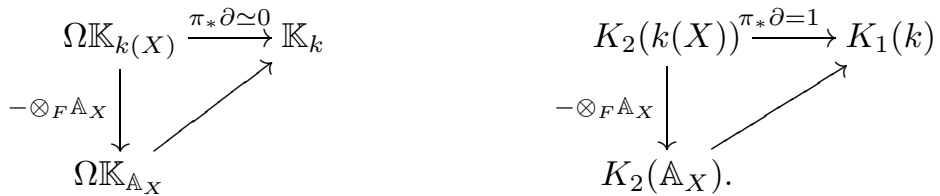
Passing to homotopy groups, we obtain the commutative diagram of abelian groups on the right. Thus, we see that $\prod_{x \in X_{0}} \pi_{*} \partial\{f, g\}=\prod_{x \in X_{0}} N_{\kappa(x) / k}(f, g)_{x}=1$, for all pairs of invertible rational functions on $X$.

Similarly one could use this result to prove reciprocity for Contou-Carrère symbols, relative to any $k$-algebra $A$. We will give more details at the end of this section, when discussing the proof of reciprocity for higher-dimensional varieties.

Theorem 7.9 (Abstract Parshin reciprocity). We denote by $\mathrm{C}$ a stable $\infty$-category, and by $\mathbf{S}_{0} \subset \mathbf{S}_{1} \subset \mathbf{C}$ a length 2 chain of localizing subcategories. The construction of (56) applied to $\left(\mathbf{S}_{1}\right)_{\widehat{(0)}} \subset \mathrm{C}_{\widehat{(0)}}$ yields a functor $G: \mathrm{C}_{\widehat{(0)}(1)} \rightarrow \mathrm{C}_{\widehat{(0,1)}}$ which belongs to a commutative diagram

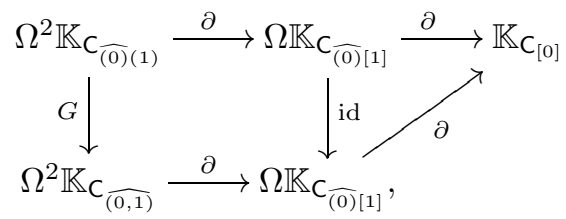

such that the composition of the top row is equivalent to the zero map.

Proof. As in the proof of Abstract Weil reciprocity, the existence of the commutative diagram follows directly from the naturality of boundary maps. We therefore turn to proving the existence of a null-homotopy for the composition of the top row. Similar to the proof of Theorem 7.7 we show that this composition factors through the juxtaposition of two subsequent maps in an exact sequence of spectra (thus is homotopic to 0 ). This is achieved by the commuting diagram below on the left:
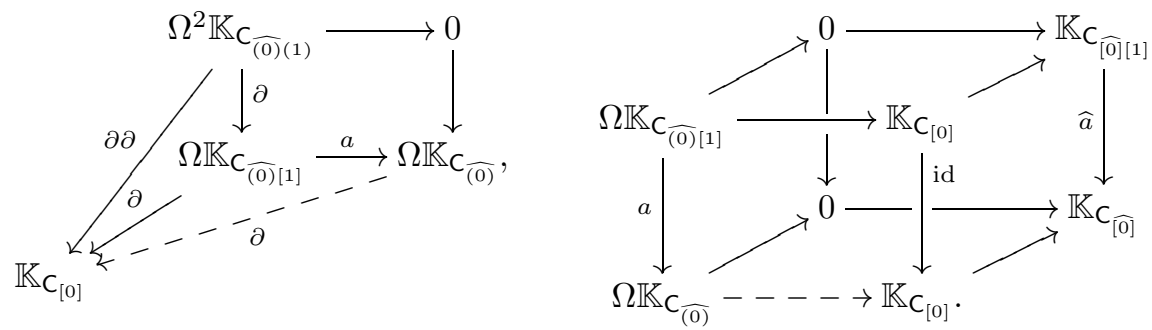

provided we can establish the existence of the dashed map. To explain the diagram, note that almost all of the maps appearing in the commutative diagram above are boundary sequences for localization sequences in algebraic $K$-theory, the exception being $a: \Omega \mathbb{K}_{\widehat{(0)}[1]} \rightarrow \Omega \mathbb{K}_{\mathrm{C}_{\widehat{(0)}}}$ which is induced by the inclusion of the localizing subcategory

$$
a: \mathrm{C}_{\widehat{(0)}[1]} \hookrightarrow \mathrm{C}_{[0]}
$$

A suitable candidate for the dashed map is given by the $K$-theory boundary morphism of the exact sequence of stable $\infty$-categories $C_{[0]} \hookrightarrow C_{\widehat{[0]}} \rightarrow C_{\widehat{(0)}}$. Naturality of boundary maps implies the existence of a commutative diagram with exact rows as depicted above on the right. Most of the arrows in the cubical diagram are not labelled. The respective maps are well-defined by the fact that the rows are localization sequences in $K$-theory. The morphism $\widehat{a}$ is induced by the inclusion of the localizing subcategory $\mathrm{C}_{\widehat{[0]}[1]}=\left(\mathbf{S}_{1}\right)_{\widehat{\mathbf{S}}_{0}} \hookrightarrow \mathrm{C}_{\widehat{\mathbf{S}}_{0}}=\mathrm{C}_{\widehat{[0]}}$ (see also Definition B.5). 
The front square of the commuting cube amounts to the existence of the commuting triangle containing the dashed map above. This concludes the proof.

Let us explain how this result implies Parshin's reciprocity statement for surfaces.

Example 7.10 (Parshin reciprocity). Let $Y$ be an integral separated excellent surface. We denote by $C$ the stable $\infty$-category $\operatorname{Perf}(Y)$. For a fixed closed point $x \in$ $Y$, we obtain a localizing subcategory $\mathbf{S}_{0}=\operatorname{Perf}_{\{x\}}(Y)$. Moreover, for every integral curve $C$, with $x \in C$, we have a localizing subcategory $\mathbf{S}_{1}=\operatorname{Perf}_{|C|}(Y)$. Theorem B.11(a) implies that $\mathrm{C}_{\widehat{(0,1)}} \cong \operatorname{Perf}\left(\mathbb{A}_{Y, C, x}\right)$, and a direct limit of the $\infty$-categories $\mathrm{C}_{\widehat{(0)}(1)}$ yields $\operatorname{Perf}\left(\operatorname{Frac}\left(\widehat{\mathcal{O}_{Y, x}}\right)\right)$. Hence, by Theorem 7.9 , we have a commutative diagram of $K$-theory groups

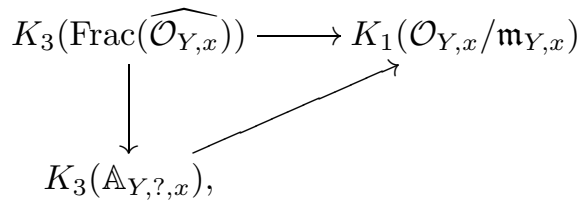

in which the top map is trivial. Here $\mathbb{A}_{Y, ?, x}$ denotes the ring of adèles for chains, $Y \supset C \supset\{x\}$, where $C$ can be an arbitrary irreducible curve containing $x$. In particular, we see that for every triple $f_{0}, f_{1}, f_{2} \in \operatorname{Frac}\left(\widehat{\mathcal{O}_{Y, x}}\right) \times$ we have the identity

$$
\prod_{C \ni x}\left(f_{0}, f_{1}, f_{2}\right)_{x \in C}=1
$$

where the product is indexed by integral closed curves containing $x$.

Combining Theorems 7.7 and 7.9, we obtain an abstract analogue of Kato reciprocity. In the next subsection we will use this result to deduce a reciprocity law for Contou-Carrère symbols.

Corollary 7.11 (Abstract Kato reciprocity). Let C be a stable $\infty$-category. We fix positive integers $i$ and $n$, and assume that we have a chain of localizing subcategories $\mathbf{S}_{j} \subset \mathrm{C}$, indexed by $0 \leq j \leq n-1$.

(a) If $i=0$, suppose that we have a commutative diagram

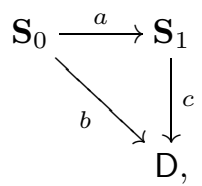

where $\mathrm{D}$ denotes a stable $\infty$-category. Then, the morphism $\Omega^{n} \mathbb{K}_{\mathrm{C}_{(0)(1, n-1)}} \rightarrow$ $\mathbb{K}_{\mathrm{D}}$ defined as the following composition

$$
\Omega^{n} \mathbb{K}_{\mathrm{C}_{(0)(1, n-1)}} \stackrel{G_{(1, n-1)}}{\longrightarrow} \Omega^{n} \mathbb{K}_{\mathrm{C}_{(0, n-1)}} \stackrel{\partial^{n}}{\rightarrow} \mathbb{K}_{\mathrm{C}_{[0]}} \stackrel{b}{\rightarrow} \mathbb{K}_{\mathrm{D}}
$$

is null-homotopic. Here, $G: \mathrm{C}_{(0)} \rightarrow \mathrm{C}_{\widehat{(0)}}$ denotes the functor of (56) applied to $\mathbf{S}_{0} \subset \mathbf{C}$, and

$$
G_{(\widehat{1, n-1})}: \mathrm{C}_{(0)(\widehat{1, n-1})} \rightarrow \mathrm{C}_{(\widehat{0, n-1})}
$$

denotes the induced functor, obtained by applying the functorial construction $(-) \underset{(1, n-1)}{ }$ to $G$. 
(b) If $i \neq 0$, then the following composition

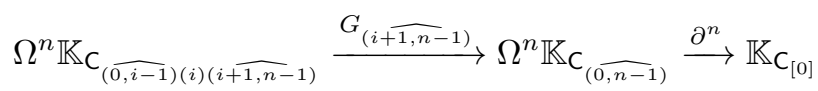

is null-homotopic. Here, $G: \mathrm{C}_{(\widehat{0, i-1})(i)} \rightarrow \mathrm{C}_{\widehat{(0, i)}}$ denotes the functor of (56) applied to the localizing subcategory $\left(\mathbf{S}_{i}\right)_{(\widehat{0, i-1)}} \subset \mathrm{C}_{(\widehat{0, i-1})}$, and

$$
G_{(i+\widehat{1, n-1)}}: \mathrm{C}_{(\widehat{0, i-1)(i)(i+1, n-1)}} \rightarrow \mathrm{C}_{(\widehat{0, n-1})}
$$

denotes the induced functor, obtained by applying the functorial construction $(-)_{(i+1, n-1)}$ to $G$.

Proof. The first assertion follows directly from Theorem 7.7 when setting $\mathbf{C}=\mathbf{S}_{1}$, and $\mathbf{S}=\mathbf{S}_{0}$.

We will now turn to the proof of the second assertion. For $j \leq i-1$ we denote by

$$
\partial_{j}: \Omega^{j+1} \mathbb{K}_{\mathrm{C}_{\widehat{(0, j)}[j+1]}} \rightarrow \Omega^{j} \mathbb{K}_{\mathrm{C}_{(\widehat{0, j-1})[j]}}
$$

the boundary morphism in $K$-theory, associated to the short exact sequence

$$
\mathrm{C}_{(\widehat{0, j-1})[j]} \hookrightarrow \mathrm{C}_{(\widehat{0, j-1}) \widehat{[j]}[j+1]} \rightarrow \mathrm{C}_{\widehat{(0, j)}[j+1]}
$$

of stable $\infty$-categories. Analogously, we have the boundary maps

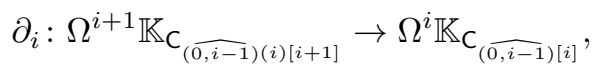

and for $j \geq i+1$

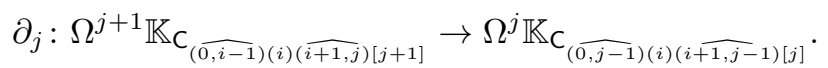

We want to show that the composition of these boundary maps satisfies $\partial_{0} \circ \cdots \circ$ $\partial_{n-1} \simeq 0$. In fact, Theorem 7.9 implies that $\partial_{i-1} \circ \partial_{i} \simeq 0$. To see this one chooses the $\mathrm{C}$ in loc. cit. to be the stable $\infty$-category $\mathrm{C}_{(\widehat{0, i-2})[i+1]}, \mathbf{S}_{0}=\mathrm{C}_{(\widehat{0, i-2})[i-1]}$, and $\mathbf{S}_{1}=\mathrm{C}_{(\widehat{0, i-2})[i]}$.

Example 7.12 (Kato reciprocity). Let $X$ be an integral separated excellent scheme of pure dimension $n$. Let $\zeta$ denote an almost saturated flag of closed integral subschemes

$$
\zeta=\left(X \supset Z_{n-1} \supset \cdots \supset Z_{i+1} \supset Z_{i-1} \supset \cdots \supset Z_{0}\right),
$$

indexed by $j \neq i$, with $\operatorname{dim} Z_{j}=j$. If $i=0$, we assume that $Z_{1}$ is proper over a field $k$. For every (not necessarily irreducible) reduced closed subscheme $Z_{i}$ of pure dimension $i$, and $Z_{i+1} \supset Z \supset Z_{i-1}$ we obtain a natural chain of localizing subcategories $\mathbf{S}_{j}:=\operatorname{Perf}_{\left|Z_{j}\right|}$ on $\mathbf{C}=\operatorname{Perf}(X)$. Abstract Kato reciprocity (Corollary 7.11) now implies the existence of a commutative diagram

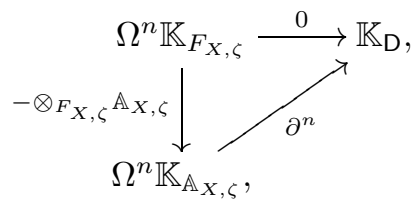

where we let $\mathrm{D}=\operatorname{Perf}(k)$ for $i=0$, and $\operatorname{Perf}_{Z_{0}}(X)$ otherwise. As before, this 
implies that for an $(n+1)$-tuple of invertible elements of $F_{X, \zeta}$, we have

$$
\prod_{Z_{i+1} \supset Z \supset Z_{i-1}}\left(f_{0}, \ldots, f_{n}\right)_{\xi_{Z}}=1
$$

where $Z$ is integral and of dimension $i$.

7.2. Reciprocity for Contou-Carrère symbols. In the following we fix a separated, reduced $k$-scheme $X$ of finite type and dimension $n$, a $k$-algebra $A$, and an integer $i$. As in Example 7.12, $\zeta$ denotes an almost saturated flag of closed integral subschemes

$$
\zeta=\left(X \supset Z_{n-1} \supset \cdots \supset Z_{i+1} \supset Z_{i-1} \supset \cdots \supset Z_{0}\right),
$$

indexed by $0 \leq j \leq n$ with $j \neq i$, and satisfying $\operatorname{dim} Z_{j}=j$. The condition of being almost saturated stipulates that up to the choice of $Z_{i+1} \supset Z_{i} \supset Z_{i-1}$, the flag cannot be further extended. If $i=0$, we assume that $Z_{1}$ is proper over a field $k$.

Alluding to the notation of abstract Kato reciprocity (Corollary 7.11), we define

$$
\mathrm{C}=\operatorname{Perf}\left(X_{A}\right), \quad \mathbf{S}_{j}=\operatorname{Perf}_{\left|\left(Z_{j}\right)_{A}\right|}\left(X_{A}\right),
$$

where $Z_{i}=Z$ is a not necessarily irreducible closed subset of pure dimension $i$, satisfying $Z_{i+1} \supset Z \supset Z_{i-1}$.

Lemma 7.13. Using the notation introduced earlier, we have the following equivalences.

(a) $\mathrm{C}_{(\widehat{0, i-1})(i)(i+1, n-1)} \cong \operatorname{Perf}\left((\mathrm{C} \circ \mathrm{L})^{n-i-1} \circ \mathrm{L} \circ(\mathrm{C} \circ \mathrm{L})^{i}\left(X_{A},\left(\xi_{Z}\right)_{A}\right)\right)$ (see Definition (7.2). In particular, taking the colimit of the diagram of these stable $\infty$-categories indexed by all possible $Z$, we obtain $\operatorname{Perf}\left(A_{\zeta}(X)\right)$.

(b) For each $Z_{i+1} \supset Z \supset Z_{i-1}$ we denote by $\xi_{Z}$ the corresponding complete flag. Then we have $\mathrm{C}_{(\widehat{0, n-1})} \cong \operatorname{Perf}\left(A_{X, \xi_{Z}}\right)$.

Proof. The second assertion is a direct consequence of Theorem B.11(c). The first assertion is proven by similar means as the results in Subsection B.2.2 as in loc. cit. one proceeds by induction, where the $i$-th step (due to the absence of completion) has to be treated separately (using Lemma B.9 instead of Corollary B.10).

Using the equivalences of stable $\infty$-categories, provided by Lemma 7.13 , abstract Kato reciprocity implies the following corollary.

Corollary 7.14 (Spectral Contou-Carrère reciprocity). The following composition

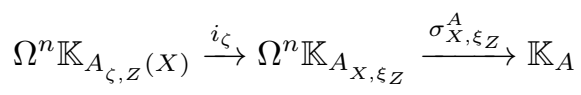

is null-homotopic (see Definition 7.2). Taking the filtered colimit over $Z$, we obtain the composition

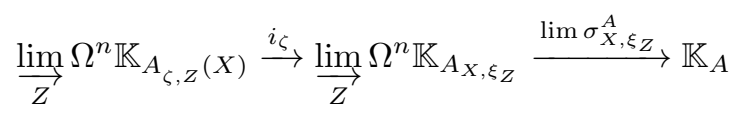

which is also null-homotopic.

Proof. Using Remark 7.3 one obtains the second commuting triangle from the first (including the null-homotopy), by taking a colimit ranging over the collection of all possible $Z_{i+1} \supset Z \supset Z_{i-1}$. At the beginning of this subsection, we have already defined a chain of localizing subcategories $\mathbf{S}_{j}$ on $\mathbf{C}=\operatorname{Perf}\left(X_{A}\right)$, which allows us to 
evoke abstract Kato reciprocity (Corollary 7.11). We only need to verify that one of the conditions (a) or (b) holds, in order to apply this result. If $i=0$, then $Z_{1}$ is proper over $k$ by assumption. By virtue of Lemma 3.15 we obtain a pushforward functor

$$
\pi_{*}: \mathbf{S}_{1} \cong \operatorname{Perf}_{\left(Z_{1}\right)_{A}}\left(X_{A}\right) \rightarrow \operatorname{Perf}(A),
$$

which yields the required commutative diagram

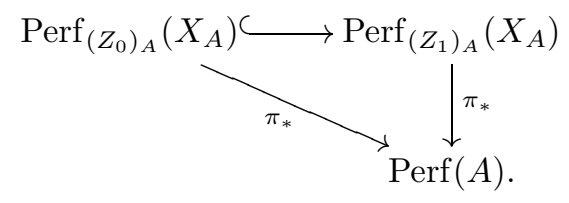

If $i \geq 1$, there is nothing to check. This concludes the proof of the first assertion.

The second assertion also follows by applying abstract Kato reciprocity (Corollary (7.11). For $j \neq i$ one defines $\mathbf{S}_{j}$ as before, and in degree $i$ one sets $\mathbf{S}_{i}=$ $\lim _{Z} \operatorname{Perf}_{Z_{A}}\left(X_{A}\right)$, where $Z$ ranges over all closed subsets which are of pure dimen$\overrightarrow{\text { sion } i}$ and satisfy $Z_{i-1} \subset Z \subset Z_{i+1}$.

Proof of Theorem 7.4. Let $f_{0}, \ldots, f_{n}$ be a commuting $(n+1)$-tuple of units in the ring $A_{\zeta}(X)$. This corresponds to a map $\Sigma_{+}^{\infty} \mathbb{T}^{n+1} \rightarrow \Sigma_{+}^{\infty}\left(B A_{\zeta}(X)^{\times}\right)$. The right hand side can be expressed as a colimit by definition of the ring $A_{\zeta}(X)$ (see Definition (7.2). Because the torus is compact, the map factors through a map $\Sigma_{+}^{\infty} \mathbb{T}^{n+1} \rightarrow \Sigma_{+}^{\infty}\left(B A_{\zeta, Z}(X)^{\times}\right)$for some $Z$.

The ring $A_{\zeta, Z}(X)$ splits into a product over the irreducible components of $Z=$ $\bigcup_{k=1}^{m} W_{k}$. Therefore, spectral Contou-Carrère reciprocity[7.14 yields a commutative diagram

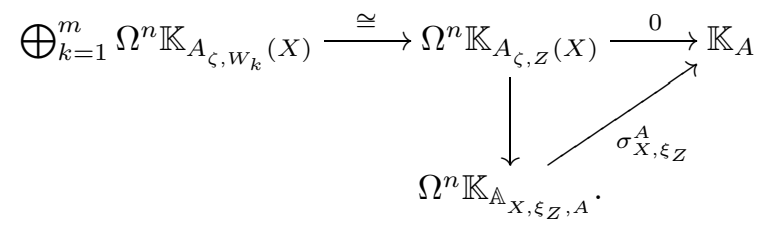

Passing to homotopy groups, and applying the resulting maps to the object represented by the Steinberg symbol $\left\{f_{0}, \ldots, f_{n}\right\}$ (i.e. a higher commutator by Proposition 4.28), we obtain the identity

$$
\prod_{i=1}^{m} \pi_{*} \partial_{W_{i}}^{n}\left\{f_{0}, \ldots, f_{n}\right\}=\prod_{i=1}^{m}\left(f_{0}, \ldots, f_{n}\right)_{\xi_{W_{i}}}=1 .
$$

This concludes the proof.

\section{Appendix A. CATEgorical AND homotopical Framework}

A.1. $\infty$-categories. We briefly review the main ideas from the theory of $\infty$-categories that are repeatedly used in our work. For a more detailed overview, we refer the reader to Groth's survey Gro10]. 
A.1.1. Spaces are $\infty$-Groupoids. The only topological spaces that play a role for us are those which are homotopy equivalent to a CW-complex. The term space (regardless of pointed or unpointed) will always refer to topological spaces of this type. Since every space $X$ is weakly equivalent to the geometric realization of the simplicial set of singular simplices $S \bullet(X)$, we could equivalently work with simplicial sets.

We now remind the reader of a hierarchy on the homotopy category of (unpointed) spaces.

- A homotopy 0-type is an unpointed space homotopy equivalent to a discrete topological space,

- a homotopy 1-type is an unpointed space with vanishing higher homotopy groups,

- a homotopy $n$-type is an unpointed space $X$ with $\pi_{k}(X)=0$ for $k \geq n+1$.

The category of homotopy 0-types is equivalent to the category of sets. The category of homotopy 1-types is closely related to the category of (small) groupoids $\mathcal{G}$. To a groupoid $\mathcal{G}$, one simply assigns the geometric realization of its nerve $|N \mathcal{G}|$. Vice versa, given an unpointed topological space $X$, we have the Poincaré groupoid $\pi_{\leq 1}(X)$. Its set of objects is the set of points in $X$. A morphism from $x \in X$ to $y \in X$ is a homotopy class of paths connecting $x$ and $y$.

The natural map of groupoids $\mathcal{G} \rightarrow \pi_{\leq 1}(|N \mathcal{G}|)$ is not a strict isomorphism. However, it is an equivalence of groupoids. Using this fact, one can show that the above functors induce an equivalence between the 2-category of groupoids and the 2 -category of homotopy 1-types. This motivates the following slogan of modern homotopy category:

The collection of homotopy $n$-types forms the $(n+1)$-category of $n$-groupoids. Unpointed spaces correspond to $\infty$-groupoids.

A.1.2. Simplicial sets and $\infty$-categories. Intuitively speaking, an $\infty$-category $C$ is a category enriched in $\infty$-groupoids (i.e. unpointed spaces). Hence, for every pair of objects $X, Y \in \mathrm{C}$ we have a space of morphisms $\operatorname{Hom}_{\mathrm{C}}(X, Y)$. Since this space will only matter up to homotopy, composition should not be expected to be defined strictly, but only up to a homotopy, which itself is well-defined up to higher homotopies of all orders. It is difficult to extract a meaningful definition from this heuristic description, but its value should not be underestimated. To a large extent it is possible to work with $\infty$-categories as a blackbox, as long as one accepts that there is a well-behaved calculus of homotopy coherent commutative diagrams.

In the rigorous setting of quasi-categories (see e.g. Lurie's Lurb]), one defines $\infty$-categories as simplicial sets satisfying a mild technical condition. This definition is motivated by the classical construction of nerves of categories. Recall that for a classical category $\mathrm{C}$ we define its nerve $N C$ to be the simplicial set with objects as 0 simplices, morphisms as 1-simplices, composable pairs of morphisms as 2-simplices, etc. Grothendieck observed that one can reconstruct a category from its nerve (even up to isomorphism of categories, see e.g. Lurb]). A simplicial set is the nerve of a category, if and only if it satisfies a collection of strict horn-filling conditions, the most important one of which is explained below. 
The set of 2-simplices of $N C$ can be understood as the set of commuting triangles as depicted below on the left:
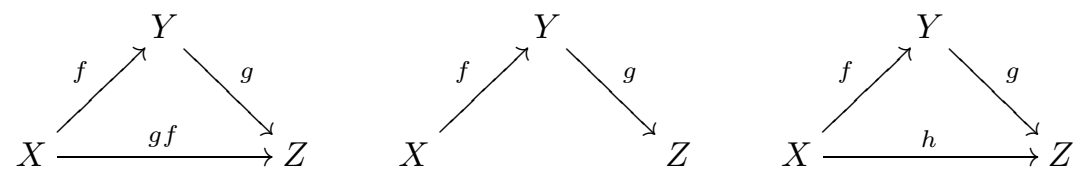

The horn-filling condition in this particular case amounts to stating that every diagram as depicted above in the middle can be completed to a commuting triangle as above. For a classical category this can always be achieved in precisely one way.

Even if one does not know the definition of an $\infty$-category, one could try to guess what the nerve of an $\infty$-category should be. Accepting the above slogan that, whatever $\infty$-categories are, we want to have a good calculus of commutative diagrams, we arrive as a definition for the set of 2 -simplices in the nerve at the set of commuting triangles as depicted above on the right. There are two interesting new features. First of all we cannot say that $h$ is the composition of $f$ and $g$. Rather, $h$ is one of possibly many compositions of $f$ and $g$. The invisible 2-cell of the triangle above should be thought of as a homotopy connecting both sides. It turns out that if we no longer require horns to be filled uniquely, this is sufficient to characterize nerves of $\infty$-categories. This is precisely how quasicategories are defined by Joyal and in Lurb.

What separates the subcategory of classical categories from its complement in quasicategories is the existence of a strict composition operation for morphisms. In $\infty$-categories, composition is only well-defined up to a contractible space of choices. It is this little bit of extra homotopical glue, which makes the theory of $\infty$-categories so flexible.

As a natural consequence of this liberality, the only possible notion of commutative diagrams is automatically homotopy coherent in a strong sense.

If $I_{\bullet}$ is a simplicial set, then an $I_{\bullet}$-indexed commutative diagram in an $\infty$ category $\mathrm{C}$ is a map of simplicial sets $I_{\bullet} \rightarrow \mathrm{C}$. A commutative square

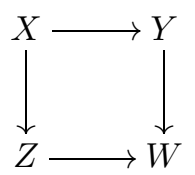

for example is a map of simplicial sets $\left(\Delta^{1}\right)^{2} \rightarrow C$, sending the 0 -simplices of the square $\left(\Delta^{1}\right)^{2}$ to the objects $X, Y, Z, W$.

A.2. Stable $\infty$-categories. We refer the reader to [Lura, Ch. 1] for a more detailed account. Every $\infty$-category $\mathrm{C}$ has an associated homotopy category $\mathrm{Ho}(\mathrm{C})$, where the set of morphisms is defined to be the set of connected components $\operatorname{Hom}_{\mathrm{Ho}(\mathrm{C})}(X, Y)=\pi_{0} \operatorname{Hom}_{\mathrm{C}}(X, Y)$. A stable $\infty$-category has a natural triangulated structure on its homotopy category. Examples include the stable $\infty$-category of spectra, and other enhancements of triangulated categories (for example pretriangulated dg-categories).

By definition, a stable $\infty$-category $C$ is pointed, i.e. there exists an initial and final object • Moreover, we assume the existence of finite limits and colimits, as 
well as that a commutative diagram

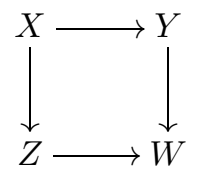

is a pullback if and only if it is a pushout. The endofunctors $\Sigma: C \rightarrow C$, and $\Omega: \mathrm{C} \rightarrow \mathrm{C}$,
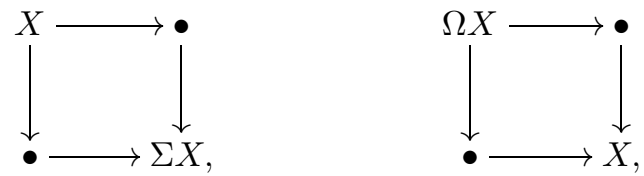

are defined by virtue of the cocartesian, respectively cartesian squares above. As a consequence of the definition of a stable $\infty$-category, $\Sigma$ and $\Omega$ are inverse equivalences. The induced functors on the homotopy category $\mathrm{Ho}(\mathrm{C})$ give rise to the translation functors of the triangulated structure of $\operatorname{Ho}(C)$. The distinguished triangles are the images of bi-cartesian squares of the form of Diagram (62) with $W=\bullet$. We denote the $\infty$-category of stable $\infty$-categories by Cat $_{\infty, s t}$.

\section{Appendix B. Derived COMpletion of schemes AND CATEGories}

"You complete me." - J. Maguire

The study of derived completion goes back to work of Greenlees-May GM92, Dwyer-Greenlees DG02, and was embedded into the realm of derived algebraic geometry by Lurie Lur09a and Gaitsgory-Rozenblyum GR14. We will mostly follow Lurie Lur09a, Ch. 4 \& 5].

For every ring $R$, and an ideal $I$, we recall (see Subsection B.1) Lurie's definition of the derived completion $\widehat{R}_{I}^{\text {der }}$. This is a connective $E_{\infty}$-ring spectrum (Lur09a, $\S 4.2])$. If $R$ is Noetherian, the derived completion is canonically equivalent to its classical counterpart [ur09a, Prop. 4.3.6]. However, for a non-Noetherian ring $R$, the derived completion is genuinely different, which affects the stable $\infty$-category of perfect complexes.

In Subsection B.2 we rephrase and generalize constructions of Efimov [Efi10]; we show how perfect complexes on the derived completions can be understood by an abstract construction on the level of stable $\infty$-categories.

We then use a calculation of Porta-Shaul-Yekutieli [PSY14] (see also [PSY15]) to conclude that $\widehat{R}_{I}^{\text {der }}$ is in fact a classical ring, if $I$ is weakly proregular in $R$ (see Definition B.3). This will allow us to remove derived rings from our work in retrospect.

B.1. Derived completion. We fix a ring $R$ and a finitely generated ideal $I$. We briefly review the notion of derived complete complexes of $R$-modules, as studied in Lur09a, §4.2]. A review of this material in the language of triangulated categories is given in [The, Tag $091 \mathrm{~N}]$. We say that a complex of $R$-modules is $I$-complete, if for every $x \in I$ the homotopy limit of the inverse system

$$
\lim [\cdots \rightarrow M \stackrel{x}{\rightarrow} M \stackrel{x}{\rightarrow} M]
$$


i.e. the fibre of

$$
\prod_{n \geq 0} M \stackrel{x}{\rightarrow} \prod_{n \geq 1} M
$$

vanishes in the stable $\infty$-category $\operatorname{DMod}(R)$. This is precisely the homotopical analogue of the condition that $x$ acts topologically nilpotently on $M$, i.e. $\bigcap_{n \in \mathbf{N}} I^{n} M=$ 0 . The resulting full subcategory of $I$-complete objects in $\operatorname{DMod}(R)$ will be denoted by $\operatorname{DMod}(R)^{I-\operatorname{comp}}$. Note that in Lur09a this subcategory is characterized differently (cf. [Lur09a, Cor. 4.2.8 \& 4.2.12]). For abstract reasons, the inclusion $\operatorname{DMod}(R)^{I \text {-comp }} \subset \operatorname{DMod}(R)$ possesses a left adjoint (see [Lur09a, Lemma 4.2.2])

$$
\widehat{(-)}^{\text {der }}: \operatorname{DMod}(R) \rightarrow \operatorname{DMod}(R)^{I-\operatorname{comp}},
$$

which will be referred to as derived completion. By Remark 4.2.6 in loc. cit. this is moreover a symmetric monoidal functor, hence we obtain an $E_{\infty}$-ring spectrum $\widehat{R}_{I}^{\mathrm{der}}$; the derived completion of $R$ at $I$.

B.2. Modification of stable $\infty$-categories. Let $X$ be a scheme, $Z$ a closed subscheme, which is defined by a locally finitely-generated sheaf of ideals. The aforementioned derived completion operation allows one to define the derived formal scheme $\widehat{X}_{Z}^{\text {der }}$ (see Lur09a, Def. 5.1.1]). If $X$ is Noetherian, it is canonically equivalent to the formal completion $\widehat{X}_{Z}$. We denote by $U$ the open complement $X \backslash Z$. Recall that $\mathrm{DQ} \operatorname{Coh}(X)$ denotes the stable $\infty$-category of complexes of quasicoherent sheaves on $X$. Pullback along the open immersion $j: U \rightarrow X$ induces a localization

$$
j^{*}: \mathrm{DQCoh}(X) \rightarrow \mathrm{DQCoh}(U) .
$$

The kernel, i.e. the full subcategory of complexes $\mathcal{F}$ satisfying $j^{*} \mathcal{F} \simeq 0$, will be denoted by $\mathrm{DQCoh}, Z(X)$. Since $j^{*} \mathcal{F} \simeq 0$ amounts to $\left.\mathcal{F}\right|_{U} \simeq 0$, it is sensible to refer to such a complex of sheaves $\mathcal{F}$ as having set-theoretic support contained in $|Z|$.

The $\infty$-category of compact objects in $\operatorname{DQCoh}_{Z}(X)$ is given by $\operatorname{Perf}_{Z}(X)$, i.e. perfect complexes on $X$ with set-theoretic support contained in $|Z|$. Moreover, $\mathrm{DQCoh}_{Z}(X)$ is compactly generated, amounting to the relation $\operatorname{DQCoh}_{Z}(X) \cong$ Ind $\operatorname{Perf}_{Z}(X)$.

Besides passing to open subschemes (localization in terms of stable $\infty$-categories), and restricting set-theoretic support (localizing subcategories), a third geometrically relevant operation is given by considering complexes of sheaves on the formal completion $\widehat{X}_{Z}^{\text {der }}$.

Quasi-coherent sheaves on the formal completion $\widehat{X}_{Z}$ are closely related to the $\infty$-category $\mathrm{DQCoh}_{Z}(X)$. In fact, we have an agreement of the full subcategories of almost connective complexes ([Lur09a, Thm. 5.1.9])

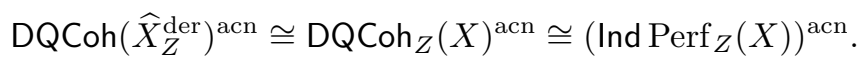

Our main interest lies in the category of perfect complexes $\operatorname{Perf}\left(\widehat{X}_{Z}^{\text {der }}\right)$ on $\widehat{X}_{Z}^{\text {der }}$. Unlike the case of a scheme, it is not sufficient to consider the full subcategory of compact objects in DQCoh $\left(\widehat{X}_{Z}^{\text {der }}\right)$ (denoted by upper script "c"). As we have seen earlier, $\mathrm{DQCoh}\left(\widehat{X}_{Z}^{\text {der }}\right)^{c} \cong \mathrm{DQCoh}_{Z}(X)^{c} \cong \operatorname{Perf}_{Z}(X)$ only yields perfect complexes with set-theoretic support contained in $|Z|$. In fact it is not very difficult to verify that structure sheaf $\mathcal{O}$ on the formal scheme $\operatorname{Spf} k[[t]]$ is not compact. 
In the remainder of this subsection we will use the observations described here to develop categorical analogues of the geometric operations given by the removal of closed subschemes and completion.

B.2.1. Completion. Let $\mathrm{C}$ be an idempotent complete stable $\infty$-category, with a full stable subcategory $\mathbf{S}$, which is idempotent complete. We refer to such an $\mathbf{S}$ simply as localizing subcategory of C. Inspired by (63) we make the following definition for the completion of $\mathbf{C}$ at $\mathbf{S}$. Proposition B.2 below compares this definition with the derived completion of rings.

Definition B.1. The completion $\mathrm{C}_{\widehat{\mathrm{S}}}$ is defined to be the idempotent closure of the essential image

$$
\operatorname{Im}[\mathrm{C} \rightarrow \operatorname{lnd} \mathbf{S}]
$$

of the functor sending $\mathcal{G} \in \mathrm{C}$ to the presheat $\mathcal{F} \mapsto \operatorname{Hom}(\mathcal{F}, \mathcal{G})$.

Note that, because the inclusion $\mathbf{S} \subset \mathbf{C}$ preserves finite colimits by assumption, the presheaf associated to $\mathcal{F} \in \mathrm{C}$ preserves colimits as well, and thus yields a well-defined functor $\mathrm{C} \rightarrow$ IndS.

Just like in Efimov's [Efi10, p. 8], we think of $C_{\widehat{\mathbf{s}}}$ as a completion on the level of Hom-spaces, not altering the class of objects. The result below can be also found in [Efi10, Remark 5.3] for Noetherian rings.

Proposition B.2. If $\mathrm{C} \cong \operatorname{Perf}(R)$, where $R$ is a ring, and $\mathbf{S}=\operatorname{Perf}_{V(I)}(X)$ for some ideal $I \subset R$, then $\operatorname{Perf}(R)_{\widehat{\mathbf{S}}} \cong \operatorname{Perf}\left(\widehat{R}_{I}^{\text {der }}\right)$.

Proof. In the following we denote by $V(I) \subset$ Spec $R$ the closed subset corresponding to the ideal $I$. We begin the proof by connecting the derived formal completion $\widehat{R}_{I}^{\text {der }}$ of Subsection B.1 with $\operatorname{Perf}_{V(I)}(R)$. Theorem 5.1.9 and Proposition 5.1.17 in Lur09a imply the existence of a commutative diagram

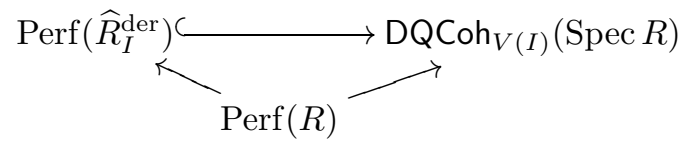

of $\infty$-categories. Using that $\mathrm{DQCoh}_{V(I)}(\operatorname{Spec} R)$ is compactly generated by $\operatorname{Perf}_{V(I)}(R)$, and the definition of $\operatorname{Perf}(R)_{\widehat{\mathbf{s}}}$ as the idempotent completion of the essential image of the functor

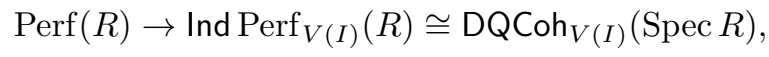

we obtain a commutative diagram

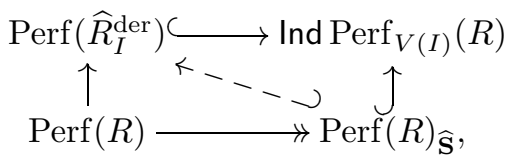

where we use the universal property of idempotent completion to produce the dashed arrow, together with the essential surjectivity of the lower horizontal functor up to idempotent completion. In order to conclude the proof, it suffices to show that we have an inclusion $\operatorname{Perf}\left(\widehat{R}_{I}^{\text {der }}\right) \subset \operatorname{Perf}(R)_{\widehat{\mathrm{s}}}$ of full subcategories of Ind $\operatorname{Perf}_{V(I)}(R)$. This follows from the fact that $\operatorname{Perf}\left(\widehat{R}_{I}^{\text {der }}\right)$ is compactly generated

\footnotetext{
${ }^{27}$ Recall that Ind(C) can be realized as the $\infty$-category of limit-preserving functors $C^{\text {op }} \rightarrow \mathrm{Sp}$.
} 
by the structure sheaf (or free module) $\mathcal{O}$, which is contained in $\operatorname{Perf}(R)_{\widehat{\mathbf{s}}}$ by the commuting diagram above.

In the result below, we use the notion of weak proregularity, which was introduced by Alonso-Jeremias-Lipman [ATJLL97] and Schenzel Sch03.

Definition B.3. Let $R$ be a ring, and $f \in R$ an element. We denote by $K(R, f)$ the Koszul complex $\left[R \rightarrow{ }^{f} R\right]$, concentrated in degrees -1 and 0 . For a tuple $\bar{f}=$ $\left(f_{0}, \ldots, f_{n}\right)$ we define the Koszul complex as $K(R, \bar{f})=\bigotimes_{i=0}^{n} K\left(R, f_{i}\right)$. An ideal $I \subset R$ is said to be weakly proregular, if there exist generators $\left(f_{0}, \ldots, f_{n}\right)$, such that for all integers $k$, the inverse system of cohomology groups $\left(H^{k}\left(K\left(R,\left(f_{0}^{i}, \ldots, f_{n}^{i}\right)\right)\right)\right)_{i}$ is pro-zero, i.e. equivalent to the zero object in the category of pro-abelian groups.

Every ideal $I$ in a Noetherian ring is weakly proregular. Moreover, the notion of weak proregularity is evidently invariant under flat base change. Hence, if $R$ is a Noetherian $k$-algebra, and $A$ is an arbitrary $k$-algebra, then the ideal $I_{A}=I \otimes_{k} A \subset$ $R_{A}=R \otimes_{k} A$ is weakly proregular.

Proposition B.4. If I is weakly proregular in $R$ (see Definition B.3), then $\widehat{R}_{I}^{\mathrm{der}} \cong$ $\widehat{R}_{I}$. In particular, we see that, for a Noetherian $k$-algebra $R$, an ideal $I$, and an arbitrary k-algebra $A$, we have ${\widehat{\left(R_{A}\right)}}_{I_{A}}^{\text {der }} \cong{\widehat{\left(R_{A}\right)}}_{I_{A}}$.

Proof. To prove this assertion we cite the main result of Porta-Shaul-Yekutieli [PSY14, Thm. 4.2]. They prove that for every perfect generator $M \in \operatorname{Perf}_{V(I)}(A)$, the so-called double centralizer is equivalent to the classical formal completion $\widehat{A}$. The double centralizer of $M$ is defined as follows. First one introduces the $E_{1}$ algebra $B=\operatorname{End}_{R}(M)$. The $E_{1}$-algebra $\operatorname{End}_{B}(M)$ is by definition the double centralizer of $M$.

We relate $\widehat{R}_{I}^{\text {der }}$ to the double centralizer by observing that by definition its underlying $E_{1}$-ring agrees with the endomorphism algebra of the image of $R$ in $\operatorname{Perf}(R)_{\widehat{V(I)}}$ :

$$
\widehat{R}_{I}^{\mathrm{der}} \cong \operatorname{End}_{\operatorname{Perf}(R)_{\widehat{V(I)}}}(R)
$$

The map $\operatorname{Perf}(R) \rightarrow \operatorname{Ind}_{\operatorname{Perf}} \operatorname{Pi}_{V(}(R)$ is given by sending a module $N$ to the presheaf $\operatorname{Hom}_{R}(-, N)$ on $\operatorname{Perf}_{V(I)}(R)$. Since $M$ is a generator, we have $\operatorname{Ind} \operatorname{Perf}_{V(I)}(R) \cong$ $\operatorname{DMod}\left(\operatorname{End}_{R}(M)^{\mathrm{op}}\right) \cong \operatorname{DMod}\left(B^{\mathrm{op}}\right)$. In particular, we see that the $R$-module $R$ is sent to $\operatorname{Hom}_{R}(M, R)=M^{\vee} \in \operatorname{DMod}(B)^{\text {op }}$. Thus, we have

$$
\operatorname{End}_{B^{\text {op }}}\left(M^{\vee}\right) \cong \operatorname{End}_{B}(M) \text {. }
$$

The right hand side is by definition the double centralizer of $M$, and therefore, by loc. cit. agrees with the classical completion $\widehat{R}$. In particular, since this is a discrete $E_{1}$-ring, this argument specifies the $E_{\infty}$-structure as well.

Since the Yoneda embedding of $\mathbf{S}$ is fully faithful, one obtains that $\mathbf{S}$ embeds fully faithfully into the formal completion $\mathrm{C}_{\widehat{\mathbf{S}}}$.

Definition B.5. Let $\mathbf{S} \subset \mathbf{T} \subset \mathrm{C}$ be a chain of localizing subcategories of $\mathrm{C}$. Then, we denote by

(a) $\mathbf{T}_{\widehat{\mathbf{S}}} \subset \mathrm{C}_{\widehat{\mathrm{S}}}$ the localizing subcategory given by the idempotent closure of the essential image $\operatorname{Im}\left[\mathbf{T} \rightarrow \mathrm{C}_{\widehat{\mathrm{S}}}\right]$, and by

(b) $\mathbf{T}_{(\mathbf{S})}$ the idempotent completion of the essential image $\operatorname{Im}[\mathbf{T} \rightarrow \mathbf{C} / \mathbf{S}]$. 
As dictated by geometric intuition, completion of $\mathbf{C}$ at $\mathbf{S}$, followed by completion at $\mathbf{T}$, yields an $\infty$-category equivalent to $\boldsymbol{C}_{\widehat{\mathbf{S}}}$. Similarly, the completion of $X$ at $Z$ should be canonically equivalent to the completion of $U$ at $Z$, if $U$ is any open subscheme containing $Z$. This is the content of the next lemma, see also Efi10, Thm. 4.1(iii)]:

\section{Lemma B.6.}

(a) Using the notation of Definition B.5, the natural map $\mathrm{C}_{\widehat{\mathbf{s}}} \cong\left(\mathrm{C}_{\widehat{\mathbf{s}}}\right)_{\widehat{\mathbf{T}_{\widehat{\mathbf{s}}}}}$ is an equivalence.

(b) Let $\mathbf{S}, \mathbf{T}$ be localizing subcategories of $\mathbf{C}$ such that for $X \in \mathbf{S}$ and $Y \in \mathbf{T}$ we have $\operatorname{Hom}_{\mathrm{C}}(X, Y) \cong 0$. We denote by $\mathrm{D}$ the idempotent completion of $\mathrm{C} / \mathbf{S}$. Then we have $\mathrm{D}_{\widehat{\mathbf{T}}} \cong \mathrm{C}_{\widehat{\mathbf{T}}}$.

Proof.

(a) By definition, the right hand side agrees with the essential image (up to idempotent completion)

$$
\operatorname{Im}\left[C_{\widehat{\mathbf{S}}} \rightarrow \operatorname{Ind} \mathbf{T}_{\widehat{\mathbf{S}}}\right]^{\text {ic }} \cong \operatorname{Im}\left[\operatorname{Im}[\mathbf{C} \rightarrow \operatorname{Ind} \mathbf{S}]^{\text {ic }} \rightarrow \operatorname{Ind}\left(\operatorname{Im}[\mathbf{T} \rightarrow \operatorname{Ind} \mathbf{S}]^{\text {ic }}\right)\right]^{\text {ic }} .
$$

The latter is equivalent to the essential image (up to idempotent completion) $\operatorname{Im}[\mathbf{C} \rightarrow \operatorname{Ind} \mathbf{S}]^{\text {ic }}$, which agrees with $C_{\widehat{\mathbf{S}}}$ by definition.

(b) At first we want to show that for $X \in \mathbf{S}$ and an arbitrary object $Y \in \mathbf{C}$ we have that the natural morphism of spaces of morphisms $\operatorname{Hom}_{\mathrm{C}}(X, Y) \rightarrow$ $\operatorname{Hom}_{\mathrm{C} / \mathbf{T}}(X, Y)$ is an equivalence. It suffices to show this for $\operatorname{Hom}_{\mathrm{Ho}(\mathrm{C})}(X, Y)$ $\rightarrow \operatorname{Hom}_{\mathrm{Ho}(\mathrm{C} / \mathbf{T})}(X, Y)$ by virtue of Whitehead's Lemma. This is a map of abelian groups, and hence we need to verify surjectivity and injectivity. A morphism $X \rightarrow^{\bar{f}} Y$ in $\mathrm{C} / \mathbf{T}$ can be represented by a zigzag $X \rightarrow Y^{\prime} \leftarrow Y$, with the right hand arrow having fibre $F$ in $\mathbf{T}$. Since we have a distinguished triangle

$$
\operatorname{Hom}_{\mathrm{C}}(X, F) \rightarrow \operatorname{Hom}_{\mathrm{C}}\left(X, Y^{\prime}\right) \rightarrow \operatorname{Hom}_{\mathrm{C}}(X, Y) \rightarrow \Sigma \operatorname{Hom}_{\mathrm{C}}(X, F)
$$

and $\operatorname{Hom}_{\mathrm{C}}(X, F) \cong 0$, since $F \in \mathbf{T}$, we see that $\operatorname{Hom}_{\mathrm{C}}(X, Y) \cong \operatorname{Hom}_{\mathrm{C}}\left(X, Y^{\prime}\right)$. A similar argument can be used to show injectivity. This shows that we have a commutative diagram of stable $\infty$-categories

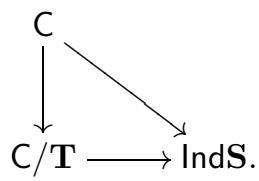

This implies that the essential images of the right-pointing functors agree, and therefore shows $\mathrm{C}_{\widehat{\mathbf{S}}} \cong(\mathrm{C} / \mathbf{T})_{\widehat{\mathbf{S}}}$.

Definition B.7. For a localizing subcategory $\mathbf{S} \subset \mathrm{C}$ we denote by $\mathrm{C}_{\widehat{(\mathbf{S})}}$ the idempotent completion of the localization $C_{\widehat{\mathbf{S}}} / \mathbf{S}$ of $\mathrm{C}_{\widehat{\mathbf{S}}}$ at $\mathbf{S}$.

This localization should be imagined as the $\infty$-category of perfect complexes on a punctured formal neighbourhood. For $X=\operatorname{Spec} R$ an affine scheme, and $Z=V(I)$ a closed subset, let $\mathbf{S}=\operatorname{Perf}_{Z}(X) \subset \operatorname{Perf}(X)$. We have $\operatorname{Perf}(X)_{\widehat{(\mathbf{s})}} \cong$ $\operatorname{Perf}\left(\operatorname{Spec} \widehat{R}_{I}^{\mathrm{der}} \backslash V(I)\right)$.

See Efimov's [Efi10, Thm. 6.1] for a global analogue of the following statement. 
Proposition B.8. Let $X$ be an excellent reduced scheme. Then the flag of localizing subcategories $\mathbf{S}_{0}, \ldots, \mathbf{S}_{n-1}$ induced by

$$
\xi: X=Z_{n} \supset Z_{n-1} \supset \cdots \supset Z_{0},
$$

where we define $\mathbf{S}_{i}=\operatorname{Perf}_{Z_{i}}(X)$, satisfies

$$
\operatorname{Perf}(X)_{(\widehat{0, n-1})} \cong \operatorname{Perf}\left(F_{X, \xi}\right),
$$

where $F_{X, \xi}$ was defined in Definition 3.9, and we use the notation of Definition 7.5 . Assume moreover that $X$ is an excellent, reduced $k$-scheme, where $k$ is a field. For every commutative $k$-algebra $A$ we have a natural equivalence

$$
\operatorname{Perf}\left(X_{A}\right)_{(\widehat{0, n-1})} \rightarrow \operatorname{Perf}\left(A_{X, \xi}\right),
$$

where $A_{X, \xi} \cong \mathbb{A}_{X_{A}}\left(\xi, \mathcal{O}_{X_{A}}\right)$.

The proof will be given in the next paragraph. Reasoning inductively, we will break the lemma down into several steps of independent interest.

B.2.2. Higher local fields via categorical completion. Recall Proposition B.2 for $R$ a ring, and an ideal $I \subset R$, the functor $\operatorname{Perf}(R) \rightarrow \operatorname{Perf}_{I}\left(\widehat{R}_{I}^{\text {der }}\right)$ induces an equivalence

$$
\operatorname{Perf}\left(\widehat{R}_{Z}^{\text {der }}\right) \rightarrow \operatorname{Perf}(R)_{\operatorname{Perf}_{V(I)}(R)} .
$$

The following Lemma uses the notion of equiheighted ideals, and localization at equiheighted ideals, which were discussed in Definition 3.6 .

Lemma B.9. Let $R$ be an excellent reduced $k$-algebra, and $I \subset R$ a radical equiheighted ideal in $R$, of height 1 . Moreover we assume that $R$ is semi-local, i.e. that the set of maximal ideals $\operatorname{Max}(R)$ is finite (therefore defining a closed subset of $\operatorname{Spec} R$ ). Then, for every $k$-algebra $A$, we have a canonical equivalence of stable $\infty$-categories

$$
\operatorname{Perf}_{V\left(I_{A}\right)}\left(\operatorname{Spec}\left(\left(R_{A}\right)_{I}\right)\right) \cong \operatorname{Perf}_{V\left(I_{A}\right)}\left(\operatorname{Spec} R_{A} \backslash \operatorname{Max}(R)\right),
$$

where $\left.\left(R_{A}\right)_{I}\right)$ denotes the ring obtained by localizing $R_{A}$ at the equiheighted ideal $I$ (see Definition 3.6). In particular, for the flag

$$
S_{0}=\operatorname{Perf}_{M a x}(R)_{A}\left(R_{A}\right) \subset \operatorname{Perf}_{V\left(I_{A}\right)}\left(R_{A}\right)
$$

the equivalence

$$
\operatorname{Perf}\left(\left(R_{A}\right)_{I}\right)_{[1]} \cong \operatorname{Perf}\left(R_{A}\right)_{(0)[1]},
$$

using the notation of Definition $\mathbf{7 . 5}$.

Proof. Let $\mathcal{U}^{\text {aff }}$ be the set of affine open subsets $\operatorname{Spec} R_{U}$ of $\operatorname{Spec} R$, containing all minimal prime ideals above $I$ (i.e. containing the generic points of $V(I) \subset \operatorname{Spec} R$ ). Inclusion of subsets induces a partial ordering on $\mathcal{U}^{\text {aff }}$. By definition, the localization $\left(R_{A}\right)_{I}$ can be expressed as the direct limit of rings $\left(R_{A}\right)_{I} \cong \lim _{U \in \mathcal{U} \text { aff }}\left(R_{U}\right)_{A}$. In particular, we obtain

$$
\operatorname{Perf}\left(\left(R_{A}\right)_{I}\right) \cong \underset{U \in \mathcal{U}^{\text {aff }}}{\lim _{\vec{f}}} \operatorname{Perf}\left(\left(R_{U}\right)_{A}\right) .
$$

The same statements are true with support condition, reading as

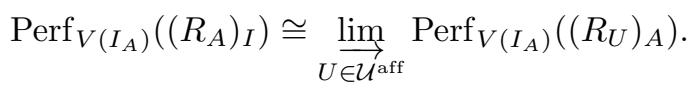


Let $\mathcal{U}$ be the set of all open subsets $U \subset \operatorname{Spec} R$, containing all minimal prime ideals above $I$. Since every open subset is a union of affine open subsets, $\mathcal{U}^{\text {aff }} \subset \mathcal{U}$ is a final directed subset. Hence we have

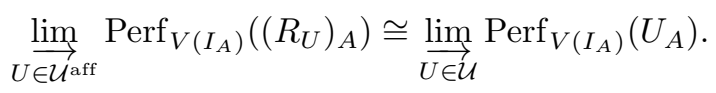

The following two observations conclude the proof:

(i) We have $\operatorname{Spec} R \backslash \operatorname{Max}(R) \in \mathcal{U}$.

(ii) All the transition maps in the inverse system computing $\lim _{U \in \mathcal{U}} \operatorname{Perf}_{V\left(I_{A}\right)}\left(U_{A}\right)$ are equivalences. In particular, we have

$$
\operatorname{Perf}_{V\left(I_{A}\right)}\left(U_{A}\right) \cong \operatorname{Perf}_{V\left(I_{A}\right)}\left(\operatorname{Spec}\left(R_{A}\right)_{I}\right)
$$

for each $U \in \mathcal{U}$.

Assertion (i) follows right from the definition of $\mathcal{U}$ : since the minimal prime ideals above $I$ are of height 1 , they cannot contain any maximal ideals. Assertion (ii) fails to hold if one does not impose the support condition. The latter ensures that, for $U \subset V \in \mathcal{U}$ with $U \cap V(I)=V \cap V(I)$, we have that restriction induces an equivalence $\operatorname{Perf}_{V\left(I_{A}\right)}\left(V_{A}\right) \rightarrow \operatorname{Perf}_{V\left(I_{A}\right)}\left(U_{A}\right)$. Since $I$ has height 1 in $R$, the open set $V(I) \backslash \operatorname{Max}(R)$ consists precisely of the generic points of $V(I)$. Therefore, every $U \in \mathcal{U}$ intersects $V(I)$ in the same open subset $V(I) \backslash \operatorname{Max}(R)$. As we have just seen this implies that all transition maps $\operatorname{Perf}_{V\left(I_{A}\right)}\left(V_{A}\right) \rightarrow \operatorname{Perf}_{V\left(I_{A}\right)}\left(U_{A}\right)$ for $U, V \in \mathcal{U}$ are equivalences. The two assertions (i) and (ii) imply now that

$$
\operatorname{Perf}_{V\left(I_{A}\right)}\left(\operatorname{Spec}\left(R_{A}\right)_{I}\right) \cong \operatorname{Perf}\left(\operatorname{Spec} R_{A} \backslash \operatorname{Max}(R)\right) .
$$

The second assertion of the Lemma is merely a reformulation, using the notation introduced in Definition 7.5 .

Corollary B.10. Let $R$ and $A$ be a k-algebras, where $R$ is assumed to be Noetherian. We denote by $R_{A}$ the tensor product $R \otimes_{k} A$. Let $I_{1} \subset I_{0} \subset R$ be a chain of equiheighted ideals, such that $I_{0}$ induces an ideal of height 1 in $R / I_{1}$ (i.e., relative codimension is 1). Using the notation of Definition 7.5 , we have a natural equivalence

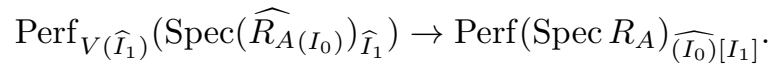

Proof. Using Lemma B.9 we obtain the vertical equivalence in the commutative diagram of stable $\infty$-categories below

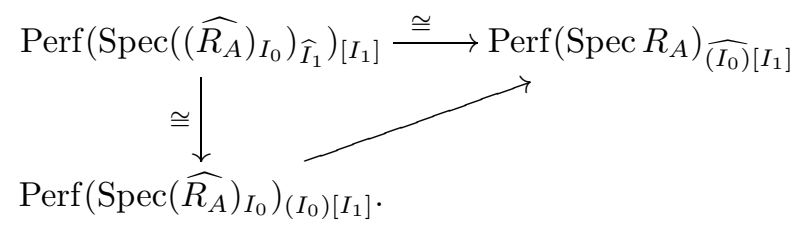

According to Definition 7.5, the $\infty$-category in the bottom left corner agrees with the localization

$$
\left(\operatorname{Perf}_{V\left(I_{1}\right)}\left(\operatorname{Spec}{\widehat{R_{A}}}_{I_{0}}^{\text {der }}\right) / \operatorname{Perf}_{V\left(I_{0}\right)}\left(\operatorname{Spec}{\widehat{R_{A}}}_{I_{0}}^{\text {der }}\right)\right)^{\mathrm{ic}} .
$$

Hence, Proposition B.2 yields the diagonal functor

$$
\left.\operatorname{Perf}\left(\operatorname{Spec} \widehat{R_{A}}\right)_{I_{0}}\right)_{\left(I_{0}\right)\left[I_{1}\right]} \rightarrow \operatorname{Perf}\left(\operatorname{Spec} R_{A}\right)_{\widehat{\left(I_{0}\right)}\left[I_{1}\right]} .
$$


Choosing an inverse for the vertical functor (well-defined up to a contractible space of choices), we obtain the required functor

$$
\operatorname{Perf}_{V\left(\widehat{I}_{1}\right)}\left(\operatorname{Spec}\left(\widehat{R}_{A}\right)_{I_{0}}\right) \rightarrow \operatorname{Perf}\left(\operatorname{Spec} R_{A}\right)_{\widehat{\left(I_{0}\right)}\left[I_{1}\right]} .
$$

Proof of Proposition B.8. We only give the proof of the second assertion, i.e. for $X$ a scheme over $k$. The first assertion is proven analogously. We may assume without loss of generality that $X$ is affine, since $Z_{0}$ is a finite union of closed points. Thus, let $R$ be a $k$-algebra, such that $X \cong \operatorname{Spec} R$.

Recall from Definition 3.9 that $A_{X, \xi}$ can be obtained by iteratively completing and localizing $R$ at a chain of equiheighted ideals $I_{0} \supset I_{1} \supset \cdots \supset I_{n-1}$, corresponding to the closed subschemes $Z_{0} \subset \cdots \subset Z_{n-1}$. We will use analogous notation for the ring

$$
A_{X, \xi}=(\mathrm{L} \circ \mathrm{C})^{n} R_{A} .
$$

The asserted equivalence is a special case of the more general statement

$$
\operatorname{Perf}_{\left(Z_{k}\right)_{A}}\left((\mathrm{~L} \circ \mathrm{C})^{k} R_{A}\right) \rightarrow \operatorname{Perf}\left(X_{A}\right)_{(\widehat{0, k-1})[k]},
$$

which will be proven inductively. Equation (65) for $k=0$ amounts to the definition of $\mathbf{S}_{0}$ :

$$
\operatorname{Perf}_{\left(Z_{0}\right)_{A}}\left(R_{A}\right) \cong \operatorname{Perf}\left(X_{A}\right)_{[0]}=\mathbf{S}_{0} .
$$

This will be the anchor point of our induction. We will prove that equation (65) holds for $k=m+1$ if it holds for $k=m$. Taking Ind-objects of both sides, and considering the (idempotent completion of the) essential image of $\operatorname{Perf}_{\left(Z_{m+1}\right)_{A}}\left(R_{A}\right) \cong$ $\operatorname{Perf}_{\left(Z_{A}\right)_{m+1}}\left(X_{A}\right)$, we see that (up to idempotent completion)

$$
\operatorname{Perf}\left((\mathrm{L} \circ \mathrm{C})^{m} R_{A}\right)_{\left[{ }_{[m]}[m+1]\right.} \cong \operatorname{Im}\left[\operatorname{Perf}_{\left(Z_{m+1}\right)_{A}}\left(R_{A}\right) \rightarrow \operatorname{Ind} \operatorname{Perf}_{\left(Z_{m}\right)_{A}}\left((\mathrm{~L} \circ \mathrm{C})^{m} R_{A}\right)\right] \text {, }
$$

here we use that for a stable $\infty$-category $\mathrm{C}$ endowed with a flag of localizing subcategories, the functor $\mathrm{C}_{[i]} \rightarrow \mathrm{C}_{\widehat{(0, i)}[i+1]}$ is essentially surjective up to idempotent completion. We have

$$
\left.\operatorname{Im}\left[\operatorname{Perf}\left(X_{A}\right)_{[m+1]} \rightarrow \operatorname{Ind} \operatorname{Perf}\left(X_{A}\right)_{(0, \widehat{m-1)}[m]}\right)\right] \cong \operatorname{Perf}\left(X_{A}\right)_{(0, \widehat{0,1}) \widehat{[m]}[m+1]},
$$

by virtue of Definition B.1. Since we are completing perfect complexes on the affine scheme $\left.\operatorname{Spec}(\mathrm{L} \circ \mathrm{C})^{m} R_{A}\right)$, Proposition B.2 gives rise to a canonical functor

$$
\operatorname{Perf}\left(\mathrm{C}(\mathrm{L} \circ \mathrm{C})^{m} R_{A}\right)_{[m+1]} \rightarrow \operatorname{Perf}\left((\mathrm{L} \circ \mathrm{C})^{m} R_{A}\right)_{\widehat{[m]}[m+1]} \cong \operatorname{Perf}\left(X_{A}\right)_{\widehat{(0, m)}[m+1]},
$$

which is an equivalence. Corollary B.10 yields an equivalence

$$
\operatorname{Perf}\left(\mathrm{L} \circ \mathrm{C}(\mathrm{L} \circ \mathrm{C})^{m} R_{A}\right)_{[m+1]} \cong \operatorname{Perf}\left(\mathrm{C}(\mathrm{L} \circ \mathrm{C})^{m} R_{A}\right)_{(m)[m+1]} .
$$

Pairing this with the functoriality of localizing at the $m$-th localizing subcategory, we therefore obtain an equivalence

$$
\operatorname{Perf}\left((\mathrm{L} \circ \mathrm{C})^{m+1} R_{A}\right)_{[m+1]} \rightarrow \operatorname{Perf}\left(R_{A}\right) \widehat{(0, m)}[m+1],
$$

of stable $\infty$-categories.

By similar techniques one proves the following:

Theorem B.11. Let $X$ be an excellent, reduced $k$-scheme of pure dimension $n$, where $k$ is a field, and $A$ a k-algebra. 
(a) We denote by $\mathbf{S}_{j} \subset \operatorname{Perf}\left(X_{A}\right)$ the localizing subcategory given by the union of the subcategories $\operatorname{Perf}_{Z_{A}}\left(X_{A}\right)$ with $\operatorname{dim} Z \leq j$. Then we have

$$
\operatorname{Perf}\left(\mathbb{A}_{X}\left(|X|_{n}^{\mathrm{red}}, \mathcal{O}_{X}\right) \widehat{\otimes}_{k} A\right) \cong \operatorname{Perf}\left(X_{A}\right)_{\widehat{(0, n)}} .
$$

(b) Let $\xi: X=Z_{n} \supset \cdots \supset Z_{i+1} \supset Z_{i-1} \supset \cdots \supset Z_{0}$ be an almost saturated flag of equiheighted closed subschemes, satisfying $\operatorname{dim} Z_{j}=j$. Let $T_{\xi} \subset|X|_{n}^{\text {red }}$ be the subset of reduced chains $\eta_{0} \prec \eta_{1} \prec \cdots \prec \eta_{n}$, such that for $j \neq i$ we have that $\eta_{j}$ is a generic point of $Z_{j}$. Then we have the equivalence $\operatorname{Perf}\left(\mathbb{A}_{X}\left(T_{\xi}, \mathcal{O}_{X}\right) \widehat{\otimes}_{k} A\right) \cong \operatorname{Perf}\left(X_{A}\right) \widehat{(0, n)}$.

Afterword B.12. Since the appearance of the preprint version of this paper on the arXiv in 2014, things have not been at a standstill. Gorchinskiy and Osipov have developed an alternative approach to a higher Contou-Carrère symbol in their series of articles GO15b, GO15a. Their methods are entirely different from ours. Moreover, Musicantov and Yom Din have independently derived a similar reciprocity law MYD17.

\section{ACKNOWLEDGMENTS}

We would like to thank T. Hausel for supporting a visit of the first and the third author to EPF Lausanne, where part of this work was carried out. We would like to thank A. Beilinson and V. Drinfeld for supporting a visit of the first and second author to the University of Chicago, where this paper was completed. The anonymous referees deserve our gratitude for their detailed reviews of our paper which led to major improvements in exposition. The third author was a guest of K. Saito at IPMU while this paper was being completed.

\section{REFERENCES}

[Ada78] John Frank Adams, Infinite loop spaces, Annals of Mathematics Studies, No. 90, Princeton University Press, Princeton, N.J.; University of Tokyo Press, Tokyo, 1978. MR505692

[ADCK89] E. Arbarello, C. De Concini, and V. G. Kac, The infinite wedge representation and the reciprocity law for algebraic curves, Theta functions-Bowdoin 1987, Part 1 (Brunswick, ME, 1987), Proc. Sympos. Pure Math., vol. 49, Amer. Math. Soc., Providence, RI, 1989, pp. 171-190. MR1013132(90i:22034)

[AGV71] M. Artin, A. Grothendieck, and J.-L. Verdier, Theorie de topos et cohomologie etale des schemas I, II, III, Lecture Notes in Mathematics, vol. 269, 270, 305, Springer, 1971.

[APR04] G. W. Anderson and F. Pablos Romo, Simple proofs of classical explicit reciprocity laws on curves using determinant groupoids over an Artinian local ring, Comm. Algebra 32 (2004), no. 1, 79-102. MR2036223 (2005d:11099)

[ATJLL97] Leovigildo Alonso Tarrío, Ana Jeremías López, and Joseph Lipman, Local homology and cohomology on schemes, Ann. Sci. École Norm. Sup. (4) 30 (1997), no. 1, 1-39, DOI 10.1016/S0012-9593(97)89914-4. MR.1422312

[Bas68] Hyman Bass, Algebraic K-theory, W. A. Benjamin, Inc., New York-Amsterdam, 1968. MR 0249491

[BBE02] Alexander Beilinson, Spencer Bloch, and Hélène Esnault, $\epsilon$-factors for Gauss-Manin determinants, Mosc. Math. J. 2 (2002), no. 3, 477-532, DOI 10.17323/1609-45142002-2-3-477-532. Dedicated to Yuri I. Manin on the occasion of his 65th birthday. MR 1988970

[BE01] Spencer Bloch and Hélène Esnault, Gauss-Manin determinants for rank 1 irregular connections on curves, Math. Ann. 321 (2001), no. 1, 15-87, DOI 10.1007/PL00004499. With an appendix in French by P. Deligne. MR1857369 
[Bei80] A. A. Běllinson, Residues and adèles (Russian), Funktsional. Anal. i Prilozhen. 14 (1980), no. 1, 44-45. MR565095

[Bei87] A. A. Beŭlinson, How to glue perverse sheaves, $K$-theory, arithmetic and geometry (Moscow, 1984), Lecture Notes in Math., vol. 1289, Springer, Berlin, 1987, pp. 42-51, DOI 10.1007/BFb0078366. MR.923134

[BGT13] Andrew J. Blumberg, David Gepner, and Gonçalo Tabuada, A universal characterization of higher algebraic K-theory, Geom. Topol. 17 (2013), no. 2, 733-838, DOI 10.2140/gt.2013.17.733. MR3070515

[BGW16a] O. Braunling, M. Groechenig, and J. Wolfson, Geometric and analytic structures on the higher adèles, Res. Math. Sci. 3 (2016), Paper No. 22, 56, DOI 10.1186/s40687016-0064-y. MR3536437

[BGW16b] O. Braunling, M. Groechenig, and J. Wolfson, Operator ideals in Tate objects, Math. Res. Lett. 23 (2016), no. 6, 1565-1631, DOI 10.4310/MRL.2016.v23.n6.a2. MR.3621099

[BGW16c] Oliver Braunling, Michael Groechenig, and Jesse Wolfson, Tate objects in exact categories, Mosc. Math. J. 16 (2016), no. 3, 433-504, DOI 10.17323/1609-4514-2016-163-433-504. With an appendix by Jan Šťovíček and Jan Trlifaj. MR3510209

[BGW18a] Oliver Braunling, Michael Groechenig, and Jesse Wolfson, The $A_{\infty}$-structure of the index map, Ann. K-Theory 3 (2018), no. 4, 581-614, DOI 10.2140/akt.2018.3.581. MR.3892960

[BGW18b] Oliver Braunling, Michael Groechenig, and Jesse Wolfson, The index map in algebraic K-theory, Selecta Math. (N.S.) 24 (2018), no. 2, 1039-1091, DOI 10.1007/s00029-0170364-0. MR3782417

[Bra18] Oliver Braunling, On the local residue symbol in the style of Tate and Beilinson, New York J. Math. 24 (2018), 458-513. MR.3855636

[Bro82] Kenneth S. Brown, Cohomology of groups, Graduate Texts in Mathematics, vol. 87, Springer-Verlag, New York-Berlin, 1982. MR672956

[CC79] Carlos E. Contou-Carrère, La jacobienne généralisée d'une courbe relative; construction et propriété universelle de factorisation (French, with English summary), C. R. Acad. Sci. Paris Sér. A-B 289 (1979), no. 3, A203-A206. MR552212

[CC81] Carlos E. Contou-Carrère, Corps de classes local géométrique relatif (French, with English summary), C. R. Acad. Sci. Paris Sér. I Math. 292 (1981), no. 9, 481-484. MR612541

[CC90] C. Contou-Carrère, Jacobiennes généralisées globales relatives (French), The Grothendieck Festschrift, Vol. II, Progr. Math., vol. 87, Birkhäuser Boston, Boston, MA, 1990, pp. 69-109. MR.1106897

[CC94] Carlos Contou-Carrère, Jacobienne locale, groupe de bivecteurs de Witt universel, et symbole modéré (French, with English and French summaries), C. R. Acad. Sci. Paris Sér. I Math. 318 (1994), no. 8, 743-746. MR 1272340

[CC13] Carlos Contou-Carrère, Jacobienne locale d'une courbe formelle relative (French, with English summary), Rend. Semin. Mat. Univ. Padova 130 (2013), 1-106, DOI 10.4171/RSMUP/130-1. MR.3148632

[Con07] Brian Conrad, Deligne's notes on Nagata compactifications, J. Ramanujan Math. Soc. 22 (2007), no. 3, 205-257. MR2356346

[Del91] P. Deligne, Le symbole modéré (French), Inst. Hautes Études Sci. Publ. Math. 73 (1991), 147-181. MR.1114212

[DG02] W. G. Dwyer and J. P. C. Greenlees, Complete modules and torsion modules, Amer. J. Math. 124 (2002), no. 1, 199-220. MR1879003

[Dri06] Vladimir Drinfeld, Infinite-dimensional vector bundles in algebraic geometry: an introduction, The unity of mathematics, Progr. Math., vol. 244, Birkhäuser Boston, Boston, MA, 2006, pp. 263-304, DOI 10.1007/0-8176-4467-9_7. MR2181808

[Efi10] A. I. Efimov, Formal completion of a category along a subcategory, arXiv:1006.4721, 062010

[Gei05] Thomas Geisser, Motivic cohomology, K-theory and topological cyclic homology, Handbook of $K$-theory. Vol. 1, 2, Springer, Berlin, 2005, pp. 193-234, DOI 10.1007/3540-27855-9_6. MR2181824 
[Gil78] Henri Antoine Gillet, The applications of algebraic K-theory to intersection theory, ProQuest LLC, Ann Arbor, MI, 1978. Thesis (Ph.D.)-Harvard University. MR.2940798

[GJ09] Paul G. Goerss and John F. Jardine, Simplicial homotopy theory, Modern Birkhäuser Classics, Birkhäuser Verlag, Basel, 2009. Reprint of the 1999 edition [MR1711612], DOI 10.1007/978-3-0346-0189-4. MR2840650

[GM92] J. P. C. Greenlees and J. P. May, Derived functors of I-adic completion and local homology, J. Algebra 149 (1992), no. 2, 438-453, DOI 10.1016/0021-8693(92)90026-I. MR 1172439

[GO15a] S. O. Gorchinskiı̆ and D. V. Osipov, Explicit formula for the higher-dimensional Contou-Carrère symbol (Russian), Uspekhi Mat. Nauk 70 (2015), no. 1(421), 183184, DOI 10.4213/rm9653; English transl., Russian Math. Surveys 70 (2015), no. 1, 171-173. MR.3353121

[GO15b] S. O. Gorchinskiŭ and D. V. Osipov, A higher-dimensional Contou-Carrère symbol: local theory (Russian, with Russian summary), Mat. Sb. 206 (2015), no. 9, 21-98, DOI 10.4213/sm8516; English transl., Sb. Math. 206 (2015), no. 9-10, 1191-1259. MR.3438594

[GR14] Dennis Gaitsgory and Nick Rozenblyum, DG indschemes, Perspectives in representation theory, Contemp. Math., vol. 610, Amer. Math. Soc., Providence, RI, 2014, pp. 139-251, DOI 10.1090/conm/610/12080. MR3220628

[Gro64] A. Grothendieck, Éléments de géométrie algébrique. III. Étude cohomologique des faisceaux cohérents. I, Inst. Hautes Études Sci. Publ. Math. 11 (1961), 167. MR217085

[Gro01] A. Grothendieck, Letter to J.-P. Serre, August 9th, 1960, Correspondance Grothendieck-Serre, Documents Mathématiques (Paris) [Mathematical Documents (Paris)], vol. 2, Société Mathématique de France, Paris, 2001. MR 1942134

[Gro10] M. Groth, A short course on infinity-categories, arXiv:1007.2925, 072010.

[Hov01] Mark Hovey, Spectra and symmetric spectra in general model categories, J. Pure Appl. Algebra 165 (2001), no. 1, 63-127, DOI 10.1016/S0022-4049(00)00172-9. MR.1860878

[HSS00] Mark Hovey, Brooke Shipley, and Jeff Smith, Symmetric spectra, J. Amer. Math. Soc. 13 (2000), no. 1, 149-208, DOI 10.1090/S0894-0347-99-00320-3. MR1695653

[Hub91] A. Huber, On the Parshin-Beĭlinson adèles for schemes, Abh. Math. Sem. Univ. Hamburg 61 (1991), 249-273, DOI 10.1007/BF02950770. MR1138291

[IR17] Florian Ivorra and Kay Rülling, K-groups of reciprocity functors, J. Algebraic Geom. 26 (2017), no. 2, 199-278, DOI 10.1090/jag/678. MR3606996

[Kap01] M. M. Kapranov, Semi-infinite symmetric powers, arXiv:math/0107089v1[math.QA], 2001.

[Kat79] Kazuya Kato, A generalization of local class field theory by using K-groups. I, J. Fac. Sci. Univ. Tokyo Sect. IA Math. 26 (1979), no. 2, 303-376. MR.550688

[Kat83] Kazuya Kato, Class field theory and algebraic K-theory, Algebraic geometry (Tokyo/Kyoto, 1982), Lecture Notes in Math., vol. 1016, Springer, Berlin, 1983, pp. 109-126, DOI 10.1007/BFb0099960. MR726423

[Kat86] Kazuya Kato, Milnor K-theory and the Chow group of zero cycles, Applications of algebraic $K$-theory to algebraic geometry and number theory, Part I, II (Boulder, Colo., 1983), Contemp. Math., vol. 55, Amer. Math. Soc., Providence, RI, 1986, pp. 241-253, DOI 10.1090/conm/055.1/862638. MR862638

[Kat00] Kazuya Kato, Existence theorem for higher local fields, Invitation to higher local fields (Münster, 1999), Geom. Topol. Monogr., vol. 3, Geom. Topol. Publ., Coventry, 2000, pp. 165-195, DOI 10.2140/gtm.2000.3.165. MR1804933

[Kel99] Bernhard Keller, On the cyclic homology of exact categories, J. Pure Appl. Algebra 136 (1999), no. 1, 1-56, DOI 10.1016/S0022-4049(97)00152-7. MR.1667558

[Ker09] Moritz Kerz, The Gersten conjecture for Milnor K-theory, Invent. Math. 175 (2009), no. 1, 1-33, DOI 10.1007/s00222-008-0144-8. MR2461425

[Ker10] Moritz Kerz, Milnor K-theory of local rings with finite residue fields, J. Algebraic Geom. 19 (2010), no. 1, 173-191, DOI 10.1090/S1056-3911-09-00514-1. MR2551760

[KS86] Kazuya Kato and Shuji Saito, Global class field theory of arithmetic schemes, Applications of algebraic $K$-theory to algebraic geometry and number theory, Part I, II (Boulder, Colo., 1983), Contemp. Math., vol. 55, Amer. Math. Soc., Providence, RI, 1986, pp. 255-331, DOI 10.1090/conm/055.1/862639. MR862639 
[KSY16] Bruno Kahn, Shuji Saito, and Takao Yamazaki, Reciprocity sheaves, Compos. Math. 152 (2016), no. 9, 1851-1898, DOI 10.1112/S0010437X16007466. With two appendices by Kay Rülling. MR.3568941

[KV07] Mikhail Kapranov and Éric Vasserot, Formal loops. II. A local Riemann-Roch theorem for determinantal gerbes (English, with English and French summaries), Ann. Sci. École Norm. Sup. (4) 40 (2007), no. 1, 113-133, DOI 10.1016/j.ansens.2006.12.003. MR2332353

[Lam68] Klaus Lamotke, Semisimpliziale algebraische Topologie (German), Die Grundlehren der mathematischen Wissenschaften, Band 147, Springer-Verlag, Berlin-New York, 1968. MR0245005

[Lod76] Jean-Louis Loday, K-théorie algébrique et représentations de groupes (French), Ann. Sci. École Norm. Sup. (4) 9 (1976), no. 3, 309-377. MR 447373

[Lura] J. Lurie, Higher algebra.

[Lurb] Jacob Lurie, Higher topos theory, Annals of Mathematics Studies, vol. 170, Princeton University Press, Princeton, NJ, 2009, DOI 10.1515/9781400830558. MR2522659

[Lur09a] J. Lurie, Derived algebraic geometry XII: Proper morphisms, completions, and the Grothendieck existence theorem, arXiv:0911.0018, 112009.

[Lur09b] J. Lurie, (infinity,2)-categories and the Goodwillie Calculus I.

[May77] J. Peter May, $E_{\infty}$ ring spaces and $E_{\infty}$ ring spectra, Lecture Notes in Mathematics, Vol. 577, Springer-Verlag, Berlin-New York, 1977. With contributions by Frank Quinn, Nigel Ray, and Jørgen Tornehave. MR0494077

[May92] J. Peter May, Simplicial objects in algebraic topology, Chicago Lectures in Mathematics, University of Chicago Press, Chicago, IL, 1992. Reprint of the 1967 original. MR:1206474

[Mil70] John Milnor, Algebraic K-theory and quadratic forms, Invent. Math. 9 (1969/70), 318-344, DOI 10.1007/BF01425486. MR260844

[Mor] M. Morrow, An introduction to higher dimensional local fields and adèles, arXiv: 1204.0586

[MVW06] Carlo Mazza, Vladimir Voevodsky, and Charles Weibel, Lecture notes on motivic cohomology, Clay Mathematics Monographs, vol. 2, American Mathematical Society, Providence, RI; Clay Mathematics Institute, Cambridge, MA, 2006. MR2242284

[MYD17] Evgeny Musicantov and Alexander Yom Din, Reciprocity laws and K-theory, Ann. K-Theory 2 (2017), no. 1, 27-46, DOI 10.2140/akt.2017.2.27. MR 3599515

[Osi05] D. V. Osipov, Central extensions and reciprocity laws on algebraic surfaces (Russian, with Russian summary), Mat. Sb. 196 (2005), no. 10, 111-136, DOI 10.1070/SM2005v196n10ABEH003710; English transl., Sb. Math. 196 (2005), no. 910, 1503-1527. MR2195664

[OZ11] Denis Osipov and Xinwen Zhu, A categorical proof of the Parshin reciprocity laws on algebraic surfaces, Algebra Number Theory 5 (2011), no. 3, 289-337, DOI 10.2140/ant.2011.5.289. MR2833793

[OZ16] Denis Osipov and Xinwen Zhu, The two-dimensional Contou-Carrère symbol and reciprocity laws, J. Algebraic Geom. 25 (2016), no. 4, 703-774, DOI 10.1090/jag/664. MR 3533184

[Pál10] Ambrus Pál, On the kernel and the image of the rigid analytic regulator in positive characteristic, Publ. Res. Inst. Math. Sci. 46 (2010), no. 2, 255-288, DOI 10.2977/PRIMS/9. MR2722779

[Par78] A. N. Paršin, Abelian coverings of arithmetic schemes (Russian), Dokl. Akad. Nauk SSSR 243 (1978), no. 4, 855-858. MR514485

[Par84] A. N. Parshin, Local class field theory: Algebraic geometry and its applications (Russian), Trudy Mat. Inst. Steklov. 165 (1984), 143-170. MR752939

[PF99] A. Parshin and T. Fimmel, Introduction to higher adelic theory (draft), unpublished, 1999.

[PR02] Fernando Pablos Romo, On the tame symbol of an algebraic curve, Comm. Algebra 30 (2002), no. 9, 4349-4368, DOI 10.1081/AGB-120013323. MR.1936475

[Pre11] Luigi Previdi, Locally compact objects in exact categories, Internat. J. Math. 22 (2011), no. 12, 1787-1821, DOI 10.1142/S0129167X11007379. MR2872533 
[PSY14] Marco Porta, Liran Shaul, and Amnon Yekutieli, Completion by derived double centralizer, Algebr. Represent. Theory 17 (2014), no. 2, 481-494, DOI 10.1007/s10468013-9405-3. MR3181733

[PSY15] Marco Porta, Liran Shaul, and Amnon Yekutieli, Erratum to: On the homology of completion and torsion, Algebras and Representation Theory 18 (2015), no. 5, 14011405 .

[Qui73] Daniel Quillen, Higher algebraic K-theory. I, Algebraic K-theory, I: Higher K-theories (Proc. Conf., Battelle Memorial Inst., Seattle, Wash., 1972), Springer, Berlin, 1973, pp. 85-147. Lecture Notes in Math., Vol. 341. MR0338129

[Saf16] Pavel Safronov, Quasi-Hamiltonian reduction via classical Chern-Simons theory, Adv. Math. 287 (2016), 733-773, DOI 10.1016/j.aim.2015.09.031. MR3422691

[Sai15] Sho Saito, On Previdi's delooping conjecture for K-theory, Algebra Number Theory 9 (2015), no. 1, 1-11, DOI 10.2140/ant.2015.9.1. MR.3317759

[Sch03] Peter Schenzel, Proregular sequences, local cohomology, and completion, Math. Scand. 92 (2003), no. 2, 161-180, DOI 10.7146/math.scand.a-14399. MR1973941

[Sch04] Marco Schlichting, Delooping the K-theory of exact categories, Topology 43 (2004), no. 5, 1089-1103, DOI 10.1016/j.top.2004.01.005. MR.2079996

[Sch06] Marco Schlichting, Negative K-theory of derived categories, Math. Z. 253 (2006), no. 1, 97-134, DOI 10.1007/s00209-005-0889-3. MR2206639

[Ser88] Jean-Pierre Serre, Algebraic groups and class fields, Graduate Texts in Mathematics, vol. 117, Springer-Verlag, New York, 1988. Translated from the French, DOI 10.1007/978-1-4612-1035-1. MR918564

[Tat68] John Tate, Residues of differentials on curves, Ann. Sci. École Norm. Sup. (4) 1 (1968), 149-159. MR227171

[The] The Stacks Project Authors, Stacks project, http://math.columbia.edu/algebraic_ geometry/stacks-git.

[TT90] R. W. Thomason and Thomas Trobaugh, Higher algebraic K-theory of schemes and of derived categories, The Grothendieck Festschrift, Vol. III, Progr. Math., vol. 88, Birkhäuser Boston, Boston, MA, 1990, pp. 247-435, DOI 10.1007/978-0-8176-45762_10. MR 1106918

[Wal85] Friedhelm Waldhausen, Algebraic K-theory of spaces, Algebraic and geometric topology (New Brunswick, N.J., 1983), Lecture Notes in Math., vol. 1126, Springer, Berlin, 1985, pp. 318-419, DOI 10.1007/BFb0074449. MR802796

[Wei94] Charles A. Weibel, An introduction to homological algebra, Cambridge Studies in Advanced Mathematics, vol. 38, Cambridge University Press, Cambridge, 1994, DOI 10.1017/CBO9781139644136. MR.1269324

[Yek92] Amnon Yekutieli, An explicit construction of the Grothendieck residue complex (English, with French summary), Astérisque 208 (1992), 127. With an appendix by Pramathanath Sastry. MR1213064

Department of Mathematics, University of Freiburg, Freiburg, Germany

Email address: oliver.braeunling@math.uni-freiburg.de

Department of Mathematics, University of Toronto, Toronto, Canada

Email address: michael.groechenig@utoronto.ca

Department of Mathematics, University of California - Irvine, Irvine, California

Email address: wolfson@uci.edu 